\title{
ON INVARIANTS OF GRAPHS WITH APPLICATIONS TO KNOT THEORY
}

\author{
KUNIO MURASUGI
}

\begin{abstract}
To each weighted graph $\Gamma$, two invariants, a polynomial $P_{\Gamma}(x, y, z)$ and the signature $\sigma(\Gamma)$, are defined. The various partial degress of $P_{\Gamma}(x, y, z)$ and $\sigma(\Gamma)$ are expressed in terms of maximal spanning graphs of $\Gamma$. Furthermore, one unexpected property of Tutte's dichromate is proved. These results are applied to knots or links in $S^{3}$.
\end{abstract}

\section{INTRODUCTION}

A graph $\Gamma$ consists of a set $V(\Gamma)$ of elements called vertices and a set $E(\Gamma)$ of elements called edges. Associated with each edge, there are either one or two vertices called its ends. An edge of $\Gamma$ is called a loop if the number of its end is 1 . We restrict ourselves to finite graphs, that is, graphs for which $V(\Gamma)$ and $E(\Gamma)$ are both finite. In this paper, however, slightly more general graphs shall be considered.

A graph $\Gamma$ is said to be weighted if either +1 or -1 , called a weight, is assigned to each edge. More precisely, $\Gamma\left(\right.$ or $\left(\Gamma, f_{\Gamma}\right)$ ) is a weighted graph if $\Gamma$ is a graph equipped with a weight function $f_{\Gamma}: E(\Gamma) \rightarrow\{1,-1\}$. For convenience, we call an edge $e$ positive if $f_{\Gamma}(e)=+1$ and negative otherwise. Since a positive graph may be considered as an unweighted (or an ordinary undirected) graph, our results can be applied to ordinary undirected graphs.

A graph $H$ is a subgraph of $\Gamma$ if $V(H) \subseteq V(\Gamma), E(H) \subseteq E(\Gamma)$, and each edge of $H$ has the same ends in $H$ as in $\Gamma$ and $H$ has induced weight function $f_{H}=f_{\Gamma} \mid E(H)$. A subgraph $H$ is a spanning subgraph of $\Gamma$ if $V(H)=V(\Gamma)$. In particular, a spanning vertex graph, denoted $V_{\Gamma}$, is the subgraph consisting of all vertices of $\Gamma$.

Now the recent unexpected discovery of close connections between a graph and a knot or link in $S^{3}$ reveals that many invariants of weighted graphs play very important roles in classical knot theory [J1, K1, K2, M3, M4, Th1, Th2].

Received by the editors June 24, 1987 and, in revised form, February 20, 1988. Presented at the Canadian Mathematical Society Winter Meeting, at Vancouver, Canada, December 12-15, 1987, organized by the Canadian Mathematical Society.

1980 Mathematics Subject Classification (1985 Revision). Primary 05C50, 57M25.

Key words and phrases. Weighted graph, polynomial of a graph, signature, Tutte's dichromate knot, link, Jones polynomial, chromatic degree, maximal spanning subgraph, alternating knot.

This research was partially supported by NSERC grant A4034. 
The main objective of this paper is to study two particularly important invariants, the polynomial $P_{\Gamma}(x, y, z)$ of $\Gamma$ (defined in (2.1)) and the signature $\sigma(\Gamma)$ of the symmetric matrix associated with $\Gamma$. (See Definition 7.2.) $P_{\Gamma}(x, y, z)$ turns out to be one of the variations of Kauffman's bracket polynomial [K1] and therefore $P_{\Gamma}(x, y, z)$ eventually induces the Jones polynomial $V_{L}(x)$ of a link $L[\mathrm{~J} 1]$. With an appropriate interpretation of $\sigma(\Gamma)$, we are able to estimate the maximal and minimal degrees of $V_{L}(x)$ (Theorem 13.3). When a knot or link is alternating, these estimates give the precise values of these degrees which finally solve many outstanding classical conjectures in knot theory (Theorems 13.4-13.8). See also [K1, K2, M3, M4, Th1, Th2].

This paper is organized as follows. $\S \S 2-6$ are mainly concerned with the polynomial of a graph $\Gamma$. The maximal and minimal degrees of $P_{\Gamma}(x, y, z)$ are given precisely in terms of the maximal positive spanning subgraph $P$ and the negative spanning subgraph $N$ in Theorem 3.1 , which is the first main theorem of this paper.

The degree of Tutte's dichromate $\chi(\Gamma)$ discussed in $\S \S 4$ and 5 is particularly interesting in connection with the last unsolved classical conjecture on alternating knots $[\mathrm{HKW}]$. We call the degree of $\chi(\Gamma)$ the chromatic degree of $\Gamma$. Using this, we define in $\S 14$ the chromatic degree $\mathbf{d}(L)$ of an alternating link $L$, which is a new geometric invariant of an alternating link. There is no algorithm to determine $\mathbf{d}(L)$. However, using the theorems proved in $\S \S 4-6$, it is possible to compute $\mathbf{d}(L)$ for many knots or links. The details will be found in Appendix (III).

In $\S 6$ we evaluate some of the coefficients of the polynomial and prove an unexpected generalization (Theorem 6.3) of the well-known fact that the leading coefficient of the chromatic polynomial is +1 [Be].

In $\S 7$ the signature of a graph is defined, and in Theorem 8.1 (the second main theorem) the best possible estimate of the signature will be proved again in terms of the spanning subgraphs $P$ and $N$.

Planar graphs are considered in $\S 9$ and formulas on these invariants for a graph and its dual will be proved. Since knot theory deals with only planar graphs, the results in $\S 9$ suggest that graph theoretical proofs will replace some sophisticated algebraic topological proofs in knot theory. (In fact, a proof of Theorem 10.1 gives us further evidence of such possibilities.)

In $\S \S 10$ and 11 we study the polynomials of two special types of graphs, called periodic graphs and congruent graphs. For applications see [M5, P1, or Tr1].

The last four sections, $\S \S 12-15$, will be devoted to applications of the results in $\S \S 2-9$ to link theory. $\S 12$ contains an alternate definition (due to Kauffman [K1]) of the Jones polynomial and an alternate proof of Thistlethwaite's theorem on the Jones polynomial of an alternating link. The conjecture mentioned earlier will be proved in $\S 13$. In $\S 14$ we briefly sketch the last Tait conjecture and prove a few relevant results. In the last section, $\S 15$, we will prove a few theorems about the writhe of a nonalternating diagram. 
Proofs of two theorems stated in $\S 9$ will be given in Appendix (I). A proof of Theorem 12.3 will be given in Appendix (II). Appendix (III) is concerned with the chromatic degree of an alternating link.

After this manuscript was completed, I found a paper by Negami [N] in which he defines a similar polynomial $f(\Gamma ; t, x, y)$ for an unweighted graph $\Gamma$. Our polynomial $P_{\Gamma}(x, y, z)$ may be considered as a generalization of his polynomial for a weighted graph. $P_{\Gamma}(x, y, z)$ determines and is determined by $f(\Gamma ; t, x, y)$ for an unweighted graph. However, there are no significant overlaps between the two papers, because Negami studied his polynomial mainly from the graph theoretical viewpoint and his main theorem is the splitting formula [N, Theorem 4.2], while we emphasize applications of our polynomials to link theory. The precise relationship between two polynomials can be found in Appendix (IV).

During the preparation of this paper I had many conversations with Dr. A. B. Lehman, to whom I would like to express my deepest appreciation. Also I wish to thank L. Kauffman, J. H. Przytycki, M. B. Thistlethwaite, and P. Traczyk for their invaluable comments.

\section{Polynomials OF GRAPHS}

Throughout this paper, what is meant by a graph is frequently the geometric realization of a graph as a finite 1-dimensional $C W$-complex in $\mathbf{R}^{3}$. A vertex and an edge correspond to a 0 -simplex and a 1 -simplex, respectively. We are free to use many terminologies from algebraic topology. For instance, the Betti number and homology group of a graph will be used in this paper. Furthermore, for two subgraphs $\Gamma_{1}$ and $\Gamma_{2}$ of $\Gamma$, the union $\Gamma_{1} \cup \Gamma_{2}$, the intersection $\Gamma_{1} \cap \Gamma_{2}$, and the complement of $\Gamma_{2}$ in $\Gamma_{1}, \Gamma_{1}-\Gamma_{2}$ are well defined as both geometric and abstract graphs.

For a set $X,|X|$ denotes the cardinality of $X$. We will use, furthermore, the following notation.

Notation. For a subgraph $G$ of $\Gamma$,

$p(G)=\left|f^{-1}(1) \cap E(G)\right|$, the number of positive edges in $G ;$

$n(G)=\left|f^{-1}(-1) \cap E(G)\right|$, the number of negative edges in $G$;

$\beta_{i}(G)$ denotes the $i$ th Betti number of $G$ as a 1-complex.

We also refer to $[\mathrm{Be}, \mathrm{Bi}$, or $\mathrm{BC}]$ for many standard terminologies in graph theory.

A graph $\Gamma$ is separable if there is a vertex $v_{0}$, called a cut vertex, such that $\beta_{0}(\Gamma)<\beta_{0}\left(\Gamma-\left\{v_{0}\right\}\right)$. Otherwise, $\Gamma$ is nonseparable.

A block is a maximal nonseparable connected subgraph of $G$. A connected graph is decomposed into finitely many blocks.

$\Gamma$ is called reduced if $\Gamma$ has neither loops nor isthmuses. An isthmus $e$ is an edge such that $\beta_{0}(\Gamma)<\beta_{0}(\Gamma-\{e\})$.

If two or more edges have the same ends, these edges are called multiple edges. A graph $\Gamma$ is called simple if $\Gamma$ has neither multiple edges nor loops. 
A two-vertex graph is called a multiple-edge graph if all edges have common (distinct) ends.

Given a graph $\Gamma$ and an edge $e$ of $\Gamma, \Gamma-(e)$ denotes the graph such that $E(\Gamma-(e))=E(\Gamma)-\{e\}$ and $V(\Gamma-(e))=V(\Gamma) . \Gamma-(e)$ is said to be obtained by deleting $e$. On the other hand, $\Gamma /(e)$ constructed from $\Gamma-(e)$ by identifying the ends of $e$ is said to be obtained by contracting $e$. If $e$ is a loop, then $\Gamma-(e)=\Gamma /(e)$.

For a weighted graph $\left(\Gamma, f_{\Gamma}\right)$, a new weighted graph $\left(\Gamma,-f_{\Gamma}\right)$ is called the opposite graph of $\left(\Gamma, f_{\Gamma}\right)$.

Now to each weighted graph $\Gamma$ we will assign a 3-variable integer polynomial $P_{\Gamma}(x, y, z)$, called the polynomial of $\Gamma . P_{\Gamma}(x, y, z)$ is one of the many variations of the polynomial originally defined by Kauffman [K1].

Definition 2.1. Let $\mathscr{S}_{\Gamma}(r, s)$ denote the set of all spanning subgraphs $G$ of $\Gamma$ such that $\beta_{0}(G)=r+1$ and $\beta_{1}(G)=s$. Then $P_{\Gamma}(x, y, z)$ is defined by

$$
P_{\Gamma}(x, y, z)=\sum_{0 \leq r, s<\infty}\left\{\sum_{G \in \mathscr{S}_{\Gamma}(r, s)} x^{p(G)-n(G)}\right\} y^{r} z^{s} .
$$

We should note that $\mathscr{S}_{\Gamma}(r, s)=\varnothing$ if $r<0$ or $r \geq|V(\Gamma)|$, or if $s<0$ or $s \geq \beta_{1}(\Gamma)+1$.

In practice, $P_{\Gamma}(x, y, z)$ can be calculated recursively using the following formulas.

(2.2) (I) If $E(\Gamma)=\varnothing$ and $|V(\Gamma)|=v$, then

$$
P_{\Gamma}(x, y, z)=y^{v-1} .
$$

(II) Let $e \in E(\Gamma)$ and $f_{\Gamma}(e)=\varepsilon$.

(i) If $e$ is not a loop, then

$$
P_{\Gamma}(x, y, z)=P_{\Gamma-(e)}(x, y, z)+x^{\varepsilon} P_{\Gamma /(e)}(x, y, z) .
$$

(ii) If $e$ is a loop, then

$$
P_{\Gamma}(x, y, z)=\left(1+x^{\varepsilon} z\right) P_{\Gamma /(e)}(x, y, z) .
$$

Proof of (2.2). (I) is obvious, since $\mathscr{S}_{\Gamma}(r, s)=\varnothing$ except for $\mathscr{S}_{\Gamma}(v-1,0)=$ $\left\{V_{\Gamma}\right\}$.

(II) Let $\mathscr{S}_{1}(r, s)=\left\{G \in \mathscr{S}_{\Gamma}(r, s) \mid G \ni e\right\}$ and $\mathscr{S}_{2}(r, s)=\left\{G \in \mathscr{S}_{\Gamma}(r, s) \mid\right.$ $G \ni e\}$. Then $\mathscr{S}_{\Gamma}(r, s)$ is the disjoint union of $\mathscr{S}_{1}(r, s)$ and $\mathscr{S}_{2}(r, s)$.

(i) Suppose that $e$ is not a loop. Then $\mathscr{S}_{1}(r, s)$ and $\mathscr{S}_{2}(r, s)$, respectively, correspond to $\mathscr{S}_{\Gamma-(e)}(r, s)$ and $\mathscr{S}_{\Gamma /(e)}(r, s)$, in one-to-one fashion. Therefore, 
we have

$$
\begin{aligned}
P_{\Gamma-(e)} & (x, y, z)+x^{\varepsilon} P_{\Gamma /(e)}(x, y, z) \\
& =\sum_{r, s} \sum_{\mathscr{S}_{\Gamma-(e)}(r, s) \ni G} x^{p(G)-n(G)} y^{r} z^{s}+\sum_{r, s} \sum_{\mathscr{S}_{\Gamma /()(}(r, s) \ni G} x^{p(G)-n(G)+\varepsilon} y^{r} z^{s} \\
& =\sum_{r, s} \sum_{\mathscr{S}_{1}(r, s) \ni G_{1}} x^{p\left(G_{1}\right)-n\left(G_{1}\right)} y^{r} z^{s}+\sum_{r, s} \sum_{\mathscr{S}_{2}(r, s) \ni G_{2}} x^{p\left(G_{2}\right)-n\left(G_{2}\right)} y^{r} z^{s} \\
& =\sum_{r, s} \sum_{\mathscr{S}_{\Gamma}(r, s) \ni G} x^{p(G)-n(G)} y^{r} z^{s} \\
& =P_{\Gamma}(x, y, z) .
\end{aligned}
$$

(ii) Suppose that $e$ is a loop. Since $\Gamma-(e)=\Gamma /(e)$, we see that $\mathscr{S}_{1}(r, s)$ and $\mathscr{S}_{2}(r, s)$, respectively, correspond to $\mathscr{S}_{\Gamma-(e)}(r, s)$ and $\mathscr{S}_{\Gamma-(e)}(r, s-1)$ in one-to-one fashion. Therefore, we have

$$
\begin{aligned}
(1+ & \left.x^{\varepsilon} z\right) P_{\Gamma /(e)}(x, y, z) \\
& =\left(1+x^{\varepsilon} z\right)\left\{\sum_{\left.r, s \mathscr{S}_{\Gamma /(e)(r, s) \ni G} x^{p(G)-n(G)} y^{r} z^{s}\right\}} x^{p(G)-n(G)} y^{r} z^{s}+\sum_{r, s} \sum_{\mathscr{S}_{\Gamma /(e)}(r, s) \ni G} x^{p(G)-n(G)+\varepsilon} y^{r} z^{s+1}\right. \\
& =\sum_{r, s} \sum_{\mathscr{S}_{\Gamma /(e)}(r, s) \ni G} x^{p\left(G_{2}\right)-n\left(G_{2}\right)} y^{r} z^{s+1} \\
& =\sum_{r, s} \sum_{\mathscr{S}_{1}(r, s) \ni G_{1}} x^{p\left(G_{1}\right)-n\left(G_{1}\right)} y^{r} z^{s}+\sum_{r, s} \sum_{\mathscr{S}_{2}(r, s+1) \ni G_{2}} x^{p(G)-n(G)} y^{r} z^{s} \\
& =\sum_{r, s} \sum_{\mathscr{S}_{\Gamma}(r, s) \ni G} \text { Q.E.D. } \\
& =P_{\Gamma}(x, y, z) . \quad \text {. }
\end{aligned}
$$

The polynomial $P_{\Gamma}(x, y, z)$ is not only an invariant of a graph but also an invariant for a 2-isomorphic graph. (For the definition of a 2-isomorphism, see [N or W].)

The following properties of $P_{\Gamma}(x, y, z)$ are easily proved.

Proposition 2.1. (1) If $\Gamma$ has $m$ connected components, $\Gamma_{1}, \Gamma_{2}, \ldots, \Gamma_{m}$, then

$$
P_{\Gamma}(x, y, z)=y^{m-1} \prod_{i=1}^{m} P_{\Gamma_{i}}(x, y, z) .
$$

(2) If $\Gamma$ is connected and if $\Gamma_{1}, \Gamma_{2}, \ldots, \Gamma_{k}$ are blocks of $\Gamma$, then

$$
P_{\Gamma}(x, y, z)=\prod_{i=1}^{k} P_{\Gamma_{i}}(x, y, z)
$$


Corollary 2.2. Let $\varepsilon_{i}=f_{\Gamma}\left(e_{i}\right)$.

(1) If $\Gamma$ consists of $k$ loops $e_{1}, \ldots, e_{k}$ then

$$
P_{\Gamma}(x, y, z)=\prod_{i=1}^{k}\left(1+x^{\varepsilon_{i}} z\right) .
$$

(2) If $\Gamma$ is a tree with $l$ edges $e_{1}, \ldots, e_{l}$, then

$$
P_{\Gamma}(x, y, z)=\prod_{i=1}^{l}\left(y+x^{\varepsilon_{i}}\right) .
$$

Corollary 2.3. If $\hat{\Gamma}$ is the opposite graph of $\Gamma$, then

$$
P_{\hat{\Gamma}}(x, y, z)=P_{\Gamma}\left(x^{-1}, y, z\right) .
$$

$P_{\Gamma}(x, y, z)$ is closely related to other polynomials which appear in graph theory. In fact, we have

Proposition 2.4. Let $\Gamma$ be a connected positive simple graph. Let $C_{\Gamma}(y)$ denote the chromatic polynomial of $\Gamma$. Then

$$
P_{\Gamma}(-1, y, 1)=C_{\Gamma}(y) y^{-1}
$$

Proposition 2.5. Let $\Gamma$ be a connected positive graph. Let $\chi(\Gamma ; y, z)$ denote Tutte's dichromate of $\Gamma[\mathrm{Tu}]$. Then

$$
P_{\Gamma}(1, y-1, z-1)=\chi(\Gamma ; y, z) .
$$

Proofs of these propositions follow immediately if we compare the recursion formulas needed to evaluate $C(y)$ or $\chi(\Gamma ; y, z)$ and (2.2) (I)-(II).

Since positive graphs are important in both graph theory and its application to knot theory, it is worth writing $P_{\Gamma}(x, y, z)$ more precisely for a positive graph $\Gamma$.

Proposition 2.6. If $\Gamma$ is a positive graph, then

$$
P_{\Gamma}(x, y, z)=x^{v-1} \sum_{0 \leq r, s<\infty}\left|\mathscr{S}_{\Gamma}(r, s)\right|\left(\frac{y}{x}\right)^{r}(x z)^{s},
$$

where $v=|V(\Gamma)|$.

Proof. Since $\Gamma$ is positive, for any spanning subgraph $G$ we have $p(G)=$ $|E(G)|$ and $n(G)=0$. Let $G \in \mathscr{S}_{\Gamma}(r, s)$. Then $r-s=\beta_{0}(G)-1-\beta_{1}(G)=$ $|V(G)|-|E(G)|-1=v-1-p(G)$, and hence $p(G)=v-1-(r-s)$. Therefore,

$$
x^{p(G)-n(G)} y^{r} z^{s}=x^{v-1}\left(\frac{y}{x}\right)^{r}(x z)^{s} .
$$

This proves Proposition 2.6.

\section{Degree of $P_{\Gamma}(x, y, z)$. The First MAIN THEOREM}

A Laurent polynomial $g\left(x_{1}, \ldots, x_{m}\right) \in \mathbb{Z}\left[x_{1}^{ \pm 1}, \ldots, x_{m}^{ \pm 1}\right]$ can be written uniquely as

$$
g\left(x_{1}, \ldots, x_{m}\right)=\sum_{-\infty<k_{1}, \ldots, k_{m}<\infty} a_{k_{1} \cdots k_{m}} x_{1}^{k_{1}} \cdots x_{m}^{k_{m}}
$$

where $a_{k_{1} \cdots k_{m}} \neq 0$. 
We define the maximal and minimal degrees of $g\left(x_{1}, \ldots, x_{m}\right)$ as follows.

$$
\begin{aligned}
& d_{\max } g\left(x_{1}, \ldots, x_{m}\right)=\max _{k_{1}, \ldots, k_{m}}\left\{k_{1}+\cdots+k_{m} \mid a_{k_{1} \cdots k_{m}} \neq 0\right\} \quad \text { and } \\
& d_{\min } g\left(x_{1}, \ldots, x_{m}\right)=\min _{k_{1}, \ldots, k_{m}}\left\{k_{1}+\cdots+k_{m} \mid a_{k_{1} \cdots k_{m}} \neq 0\right\} .
\end{aligned}
$$

In this section, we determine $d_{\max }$ and $d_{\min }$ for $P_{\Gamma}(x, y, z)$.

Let $\Gamma$ be a (weighted) graph. We reserve $P$ and $N$, exclusively, for the maximal positive and negative spanning subgraphs of $\Gamma$ respectively. In other words, $P$ and $N$ are the spanning subgraphs such that $E(P)=f_{\Gamma}^{-1}(1)$ and $E(N)=f_{\Gamma}^{-1}(-1)$.

A spanning subgraph $G$ belongs to some $\mathscr{S}_{\Gamma}(r, s)$. For convenience, we say $G \in \mathscr{S}_{\Gamma}\left(r_{G}, s_{G}\right)$, i.e., $r_{G}=\beta_{0}(G)-1$ and $s_{G}=\beta_{1}(G)$.

Theorem 3.1 (First Main Theorem). Let $\Gamma$ be a graph. Then

$$
\begin{aligned}
& d_{\max } P_{\Gamma}(x, y, z)=p(\Gamma)+r_{P}+s_{P} \\
& d_{\min } P_{\Gamma}\left(x, y^{-1}, z^{-1}\right)=-\left(n(\Gamma)+r_{N}+s_{N}\right) .
\end{aligned}
$$

Proof. First we show that it suffices to prove the theorem for a connected graph. In fact, if $\Gamma$ has $m$ connected components $\Gamma_{1}, \ldots, \Gamma_{m}$, then $P_{\Gamma}(x, y, z)=$ $y^{m-1} \prod_{i=1}^{m} P_{\Gamma_{i}}(x, y, z)$. Noting that $r_{P}=\sum_{i=1}^{m} r_{P_{i}}+(m-1)$, where $P_{i}$ denotes the maximal positive graph of $\Gamma_{i}$, we have

$$
\begin{aligned}
d_{\max } P_{\Gamma}(x, y, z) & =m-1+\sum_{i=1}^{m} d_{\max } P_{\Gamma_{i}}(x, y, z) \\
& =m-1+\sum_{i=1}^{m}\left\{p\left(\Gamma_{i}\right)+r_{P_{t}}+s_{P_{i}}\right\} \\
& =m-1+p(\Gamma)+\sum_{i=1}^{m} r_{P_{i}}+\sum_{i=1}^{m} s_{P_{i}} \\
& =p(\Gamma)+r_{P}+s_{P} .
\end{aligned}
$$

This proves $(3.2)(1)$. Similarly, $(3.2)(2)$ holds for a disconnected graph if it is proved for a connected graph. Therefore, we assume henceforth that $\Gamma$ is connected.

Second, we note that $P_{\Gamma}(x, y, z)$ and $P_{\Gamma}\left(x, y^{-1}, z^{-1}\right)$ are positive polynomials; i.e., all nonzero coefficients are positive.

Now, to each spanning subgraph $G$ of $\Gamma$, there is associated a term $x^{p(G)-n(G)} y^{r_{G}} z^{s_{G}}$ in $P_{\Gamma}(x, y, z)$ and $x^{p(G)-n(G)} y^{-r_{(i}} z^{-s_{i}}$ in $P_{\Gamma}\left(x, y^{-1}, z^{-1}\right)$. For convenience, the degrees of these terms are called the degree of $G$ and are denoted by $\operatorname{deg} G=p(G)-n(G)+r_{G}+s_{G}$ and $\operatorname{deg}^{*} G=p(G)-n(G)-r_{G}-s_{G}$. Since $\operatorname{deg} P=p(\Gamma)+r_{P}+s_{P}$ and $\operatorname{deg}^{*} N=-n(\Gamma)-r_{N}-s_{N}$, we only need to 
show that for any spanning subgraph $G$,

(1) $\operatorname{deg} G \leq \operatorname{deg} P$, and

(2) $\operatorname{deg}^{*} G \geq \operatorname{deg}^{*} N$.

We use the following lemma.

Lemma 3.2. For any spanning subgraph $G$ of $\Gamma$,

$$
\begin{array}{ll}
\text { (1) } & r_{G}-\{p(\Gamma)-p(G)\} \leq r_{p} \text { and } s_{G}-n(G) \leq s_{P} \\
\text { (2) } & r_{G}-\{n(\Gamma)-n(G)\} \leq r_{N} \text { and } s_{G}-p(G) \leq s_{N}
\end{array}
$$

Proof. Since $P$ contains all positive edges in $\Gamma$, any positive edge in $G$ is contained in $P$. Therefore, $G$ is obtained from $P$ by removing $p(\Gamma)-p(G)$ positive edges and then by adding $n(G)$ negative edges. Let $H$ be a spanning subgraph of $\Gamma$ and $H^{\prime}$ be the subgraph obtained from $H$ either by removing one edge or by adding one edge. Then we see easily that

$$
\left|r_{H}-r_{H^{\prime}}\right| \leq 1 \text { and }\left|s_{H}-s_{H^{\prime}}\right| \leq 1 \text {, }
$$

and hence

$$
r_{G} \leq r_{P}+p(\Gamma)-p(G) \text { and } s_{G} \leq s_{P}+n(G)
$$

This proves (3.4)(1). A proof of (2) is analogous but by taking $P$ instead of $N$ and hence is omitted.

Now, using Lemma 3.2, we have

$$
\begin{aligned}
\operatorname{deg} P & =p(\Gamma)+r_{P}+s_{P} \geq p(G)+r_{G}+s_{G}-n(G)=\operatorname{deg} G, \quad \text { and } \\
\operatorname{deg}^{*} N & =-n(\Gamma)-r_{N}-s_{N} \leq-n(G)-r_{G}+p(G)-s_{G}=\operatorname{deg}^{*} G .
\end{aligned}
$$

This proves (3.3) and hence Theorem 3.1. Q.E.D.

When $\Gamma$ is reduced, we can slightly strengthen Theorem 3.1 to Theorem 3.3 below. Theorems 3.3 and 3.4 are of fundamental importance for applications to link theory.

Theorem 3.3. If $\Gamma$ is a reduced positive graph, then for any spanning subgraph $G$, not $P$ or $N$,

(1) $\operatorname{deg} G \supsetneqq \operatorname{deg} P$;

(2) $\operatorname{deg}^{*} G \supsetneqq \operatorname{deg}^{*} N$.

Proof. We may assume without loss of generality that $\Gamma$ is connected.

(1) Since $\Gamma$ is positive, $P=\Gamma$ and $N=V_{\Gamma}$, and hence $n(\Gamma)=0, r_{P}=0$, $r_{N}=|V(\Gamma)|-1$, and $s_{N}=0$. Therefore, we have from (3.4) $s_{G} \leq s_{P}$ and $s_{G}-p(G) \leq 0$. Using these inequalities, we have $\operatorname{deg} G=p(G)+r_{G}+s_{G} \leq$ $p(G)+r_{G}+s_{P}$ and $\operatorname{deg} P=p(\Gamma)+s_{P}$ and, moreover, $\operatorname{deg}^{*} G=p(G)-r_{G}-s_{G} \geq$ $-r_{G}$ and $\operatorname{deg}^{*} N=-r_{N}-s_{N}=-\{|V(\Gamma)|-1\}$. Therefore, it suffices to show that

(1) $\quad p(G)+r_{G} \lesseqgtr p(\Gamma)$, and

(2) $\quad r_{G} ફ|V(\Gamma)|-1$. 
Let $p(\Gamma)-p(G)=q \geq 1$. Then $G$ is obtained from $P(=\Gamma)$ by removing $q$ edges, $e_{1}, e_{2}, \ldots, e_{q}$ say. Let $\Gamma_{j}=\Gamma-\left\{e_{1}, e_{2}, \ldots, e_{j}\right\}, 0 \leq j \leq q$. Note that $\Gamma_{0}=P$ and $\Gamma_{q}=G$. Since $\Gamma$ has no isthmuses, $\Gamma_{1}$ is still connected, i.e., $r_{\Gamma_{1}}=0$. Inductively, we can prove that $r_{\Gamma_{j}} \leq j-1$ for $1 \leq j \leq q$ and, hence, $r_{G}=r_{\Gamma_{q}} \leq q-1=p(\Gamma)-p(G)-1$. This implies that $p(G)+r_{G}<p(\Gamma)$, which proves (3.6)(1).

To prove (3.6)(2), we note that $N$ is the spanning vertex graph $V_{\Gamma}$ and $G$ is obtained from $N$ by adding $p(G)$ positive edges, $e_{1}^{\prime}, \ldots, e_{p(G)}^{\prime}$, say. Let $N_{j}=N \cup\left\{e_{1}^{\prime}, \ldots, e_{j}^{\prime}\right\}, 0 \leq j \leq p(G)$. Note that $N_{0}=N$ and $N_{p(G)}=G$, and $p(G) \geq 1$. Since $N$ does not have a loop, $e_{1}^{\prime}$ is not a loop and hence $r_{N_{1}}=|V(\Gamma)|-2$ and, inductively, we can prove that $r_{N_{j}} \leq|V(\Gamma)|-j-1$ for $1 \leq j \leq p(G)$. Therefore, $r_{G}=r_{N_{p(G)}} \leq|V(\Gamma)|-p(G)-1 \lesseqgtr|V(\Gamma)|-1$. This proves (3.6)(2). A proof of Theorem 3.3 is now complete. Q.E.D.

Theorem 3.4. Let $\Gamma$ be a connected graph. Let $G$ be a spanning subgraph of $\Gamma$ and $G^{\prime}$ be the spanning subgraph such that $E\left(G^{\prime}\right)=E(\Gamma)-E(G) .\left(G^{\prime}\right.$ is called the spanning complement of $G$.) Then

$$
r_{G}+s_{G}+r_{G^{\prime}}+s_{G^{\prime}} \leq|E(\Gamma)| .
$$

Furthermore, equality holds in (3.7) iff $G$ is a union of some (not necessarily all) blocks of $\Gamma$ (and hence $G^{\prime}$ is a union of the remaining blocks).

Proof. Use the Mayer-Vietoris homology sequence:

$$
\begin{aligned}
& \rightarrow H_{1}\left(G \cap G^{\prime}\right) \rightarrow H_{1}(G) \oplus H_{1}\left(G^{\prime}\right) \stackrel{i_{\rightarrow}}{\rightarrow} H_{1}\left(G \cup G^{\prime}\right) \\
& \rightarrow H_{0}\left(G \cap G^{\prime}\right) \rightarrow H_{0}(G) \oplus H_{0}\left(G^{\prime}\right) \rightarrow \cdots
\end{aligned}
$$

Since $G \cap G^{\prime}=V_{\Gamma}$, it follows that $H_{1}\left(G \cap G^{\prime}\right)=0$ and $i_{*}$ is a monomorphism. Since every homology group is free abelian, we see that $\beta_{1}(G)+\beta_{1}\left(G^{\prime}\right)=s_{G}+$ $s_{G^{\prime}} \leq \beta_{1}\left(G \cup G^{\prime}\right)=|E(\Gamma)|-\{|V(\Gamma)|-1)$. Since $r_{G}+1-s_{G}=|V(G)|-|E(G)|$ for any spanning subgraph $G$, it follows that $r_{G}+s_{G}+r_{G^{\prime}}+s_{G^{\prime}}=s_{G}-|E(G)|+$ $|V(\Gamma)|-1+s_{G}+s_{G^{\prime}}-\left|E\left(G^{\prime}\right)\right|+|V(\Gamma)|-1+s_{G^{\prime}}=2\left(s_{G}+s_{G^{\prime}}\right)-|E(\Gamma)|+2|V(\Gamma)|-2 \leq$ $2(|E(\Gamma)|-|V(\Gamma)|+1)-|E(\Gamma)|+2|V(\Gamma)|-2=|E(\Gamma)|$.

Furthermore, equality holds in (3.7) iff $i_{*}$ is an isomorphism, and hence $G$ must be a union of blocks of $\Gamma$. This completes a proof of Theorem 3.4. Q.E.D.

\section{Chromatic Degree of A GRAPH}

Since the polynomial $P_{\Gamma}(x, y, z)$ has three variables, we can define various partial degres of $P_{\Gamma}(x, y, z)$, for example, the degree of $P_{\Gamma}(x, y, z)$ with respect to $x, y$, or $z$. However, most of these partial degrees are not interesting. In fact, we observe:

Proposition 4.1.

(1) $d_{\max } P_{\Gamma}(x, 1,1)=p(\Gamma)$ and $d_{\min } P_{\Gamma}(x, 1,1)=-n(\Gamma)$. 
(2) $d_{\max } P_{\Gamma}(1, y, 1)=r(\Gamma)$ and $d_{\min } P_{\Gamma}(1, y, 1)=0$.

(3) $d_{\max } P_{\Gamma}(1,1, z)=s(\Gamma)$ and $d_{\min } P_{\Gamma}(1,1, z)=0$.

One of the interesting and important partial degrees of $P_{\Gamma}(x, y, z)$ is $d_{\max } P_{\Gamma}(1, y, z)$, which will be discussed in this section and the next.

Since $P_{\Gamma}(1, y-1, z-1)=\chi(\Gamma ; y, z)$ (Proposition 2.5), $d_{\max } P_{\Gamma}(1, y, z)$ is nothing more than the degree of Tutte's dichromate. We call it the chromatic degree of $\Gamma$ in this paper. (See Definition 4.1 below.) There is very little literature relevant to this degree. Theorems $4.5-4.8$ below are probably the first general theorems about the chromatic degree.

Although there is an algorithm to determine the chromatic degree, it seems quite difficult to describe it precisely, contrary to $d_{\max } P_{\Gamma}(x, y, z)$. We are only able to determine the degree for certain special types of graphs.

Let $\Gamma$ be a graph. Since $d_{\max } P_{\Gamma}(1, y, z)$ does not depend on the weight, we henceforth assume that $\Gamma$ is a positive graph. Let $G$ be a spanning subgraph of $\Gamma$.

Definition 4.1. The chromatic degree of $G, d(G)$, is $d(G)=r_{G}+s_{G}$, and the chromatic degree of $\Gamma, \mathbf{d}(\Gamma)$ is

$$
\begin{aligned}
\mathbf{d}(\Gamma)= & \max d(G) \\
& G: \text { spanning subgraphs } .
\end{aligned}
$$

\section{Proposition 4.2.}

$$
\mathbf{d}(\Gamma)=d_{\max } P_{\Gamma}(1, y, z)=d_{\max } \chi(\Gamma ; y, z) .
$$

$\mathbf{d}(\Gamma)$ is recursively determined by the following

\section{Theorem 4.3.}

(1) If $\Gamma_{1}, \ldots, \Gamma_{m}$ are connected components of $\Gamma$, then

$$
\mathbf{d}(\Gamma)=\sum_{i=1}^{m} \mathbf{d}\left(\Gamma_{i}\right)+m-1 .
$$

(2) If $\Gamma_{1}, \ldots, \Gamma_{k}$ are blocks of $\Gamma$, then

$$
\mathbf{d}(\Gamma)=\sum_{i=1}^{k} \mathbf{d}\left(\Gamma_{i}\right) .
$$

(3) If $\Gamma$ consists of $l$ loops and $k$ isthmuses, then $\mathbf{d}(\Gamma)=l+k$.

(4) If $E(\Gamma)=\varnothing$ and $|V(\Gamma)|=v$, then $\mathbf{d}(\Gamma)=v-1$.

(5) Suppose that $\Gamma$ is connected and $E(\Gamma) \neq \varnothing$. Let $e \in E(\Gamma)$. If $e$ is neither a loop nor an isthmus, then

$$
\mathbf{d}(\Gamma)=\max \{\mathbf{d}(\Gamma-(e)), \mathbf{d}(\Gamma /(e))\} .
$$


Proof. (1)-(4) follow from Proposition 2.1 and Corollary 2.2. (5) follows from the fact that $P_{\Gamma}(1, y, z)=P_{\Gamma-(e)}(1, y, z)+P_{\Gamma /(e)}(1, y, z)$ and $P_{\Gamma}(1, y, z)$ is a positive polynomial.

A spanning subgraph $G$ of $\Gamma$ is called maximal if $d(G)=\mathbf{d}(\Gamma)$. A maximal spanning subgraph need not be unique.

Proposition 4.4. For any subgraph $\Gamma_{0}$ of $\Gamma$,

$$
\mathbf{d}(\Gamma) \geq \mathbf{d}\left(\Gamma_{0}\right)+|V(\Gamma)|-\left|V\left(\Gamma_{0}\right)\right| .
$$

Proof. Let $G_{0}$ be a maximal spanning subgraph of $\Gamma_{0}$. Let $v_{1}, v_{2}, \ldots, v_{k}$ be the elements in $V(\Gamma)-V\left(\Gamma_{0}\right)$. Then $G_{0} \cup\left\{v_{1}, v_{2}, \ldots, v_{k}\right\}$ is a (not necessarily maximal) spanning subgraph of $\Gamma$, and hence

$$
\mathbf{d}(\Gamma) \geq d\left(G_{0} \cup\left\{v_{1}, v_{2}, \ldots, v_{k}\right\}\right)=d\left(G_{0}\right)+k=\mathbf{d}\left(\Gamma_{0}\right)+k \text {. Q.E.D. }
$$

Suppose that a graph $\Gamma$ is a union of two subgraphs $\Gamma_{1}$ and $\Gamma_{2}$ which meet in $m$ common vertices $v_{1}, v_{2}, \ldots, v_{m}$. Let $U=\left\{v_{1}, v_{2}, \ldots, v_{m}\right\}$ and denote by $\Gamma_{2} / U$ the graph obtained from $\Gamma_{2}$ by identifying all vertices in $U$ to a single vertex. If $m=1$, then $\Gamma_{2} / U=\Gamma_{2}$.

For any maximal spanning subgraph $G$ of $\Gamma, G \cap \Gamma_{1}$ and $G \cap \Gamma_{2}$ are spanning subgraphs of $\Gamma_{1}$ and $\Gamma_{2}$, respectively, and therefore we have

$$
\mathbf{d}(\Gamma) \leq \mathbf{d}\left(\Gamma_{1}\right)+\mathbf{d}\left(\Gamma_{2}\right) .
$$

(4.5) can be generalized to (4.6) below.

\section{Theorem 4.5.}

$$
\mathbf{d}(\Gamma) \leq \mathbf{d}\left(\Gamma_{1}\right)+\mathbf{d}\left(\Gamma_{2} / U\right) .
$$

Proof. A proof will be given by induction on $m$. Since (4.6) becomes (4.5) when $m=1$, we may assume that $m \geq 2$.

Take a maximal spanning subgraph $G$ of $\Gamma$. Suppose first that $G$ contains some vertex, $v_{m}$ say, as an isolated vertex. Then $d\left(G-\left\{v_{m}\right\}\right)=d(G)-1$. Now remove all edges incident to $v_{m}$ from $\Gamma$ to obtain the subgraph $\Gamma^{\prime}$. Since $v_{m}$ is an isolated vertex in $G, G \cap \Gamma^{\prime}=G^{\prime}$ is also maximal. Let $\Gamma_{i}^{\prime}=\Gamma_{i} \cap \Gamma^{\prime}$ $(i=1,2)$. Then $\Gamma^{\prime}=\Gamma_{1}^{\prime} \cup \Gamma_{2}^{\prime}$ and $\Gamma_{1}^{\prime} \cap \Gamma_{2}^{\prime}=\left\{v_{1}, v_{2}, \ldots, v_{m-1}\right\}$ and the induction assumption yields

$$
\mathbf{d}\left(\Gamma^{\prime}\right) \leq \mathbf{d}\left(\Gamma_{1}^{\prime}\right)+\mathbf{d}\left(\Gamma_{2}^{\prime} /\left\{v_{1}, \ldots, v_{m-1}\right\}\right) .
$$

Since $\Gamma_{1}^{\prime}$ is a subgraph of $\Gamma_{1}$ and $\left|V\left(\Gamma_{1}\right)\right|=\left|V\left(\Gamma_{1}^{\prime}\right)\right|+1$, it follows from (4.4) that $\mathbf{d}\left(\Gamma_{1}^{\prime}\right)+1 \leq \mathbf{d}\left(\Gamma_{1}\right)$. Furthermore, $\Gamma_{2}^{\prime} /\left\{v_{1}, v_{2}, \ldots, v_{m-1}\right\}$ is a subgraph of $\Gamma_{2} / U$, and hence $\mathbf{d}\left(\Gamma_{2}^{\prime} /\left\{v_{1}, v_{2}, \ldots, v_{m-1}\right\}\right) \leq \mathbf{d}\left(\Gamma_{2} / U\right)$. Therefore we have from (4.7)

$$
d(G)-1=\mathbf{d}(\Gamma)-1=\mathbf{d}\left(\Gamma^{\prime}\right) \leq \mathbf{d}\left(\Gamma_{1}^{\prime}\right)+\mathbf{d}\left(\Gamma_{2} / U\right)
$$

and

$$
\mathbf{d}(\Gamma) \leq \mathbf{d}\left(\Gamma_{1}^{\prime}\right)+1+\mathbf{d}\left(\Gamma_{2} / U\right) \leq \mathbf{d}\left(\Gamma_{1}\right)+\mathbf{d}\left(\Gamma_{2} / U\right),
$$


which proves (4.6). This argument is also valid if some vertex, $v_{m}$ say, in $U$ is isolated in $G_{1}$ (or in $G_{2}$ ); i.e., there are no edges in $G_{1}$ (or in $G_{2}$ ) which are incident to $v_{m}$. Therefore, we may assume hereafter that none of the vertices in $U$ is isolated in $G_{1}$ and $G_{2}$. For simplicity, we write $\Gamma_{2} / U=\hat{\Gamma}_{2}$ and $G_{2} / U=\hat{G}_{2} \cdot \hat{G}_{2}$ is a subgraph of $\hat{\Gamma}_{2}$. Let $C_{1}, C_{2}, \ldots, C_{s}$ be basic circuits in $G$; i.e., $\left\{C_{1}, C_{2}, \ldots, C_{s}\right\}$ forms a basis for $H_{1}(G ; \mathbf{Z})$. Suppose that each $C_{i}$ belongs to either $\Gamma_{1}$ or $\Gamma_{2}$, but not both. Since $\beta_{1}\left(\hat{G}_{2}\right) \geq \beta_{1}\left(G_{2}\right), \beta_{1}(G) \leq$ $\beta_{1}\left(G_{1}\right)+\beta_{1}\left(\hat{G}_{2}\right)$. To evaluate $\beta_{0}\left(G_{i}\right)$, consider the smallest subgraphs $G_{i}^{\prime}$ of $G_{i}$ which contain $U$. Let $n_{i}=\beta_{0}\left(G_{i}^{\prime}\right)$. Then we see easily that

$$
\beta_{0}(G)=\beta_{0}\left(G_{1}\right)+\beta_{0}\left(G_{2}\right)-n_{1}-n_{2}+1 .
$$

Since $\beta_{0}\left(G_{2}\right)-n_{2}=\beta_{0}\left(\hat{G}_{2}\right)-1$, we have

$$
\begin{aligned}
\mathbf{d}(\Gamma) & =d(G)=\beta_{0}(G)+\beta_{1}(G)-1 \\
& \leq \beta_{0}\left(G_{1}\right)+\beta_{0}\left(\hat{G}_{2}\right)-n_{1}+\beta_{1}\left(G_{1}\right)+\beta_{1}\left(\hat{G}_{2}\right)-1 \\
& \leq\left\{\beta_{0}\left(G_{1}\right)+\beta_{1}\left(G_{1}\right)-1\right\}+\left\{\beta_{0}\left(\hat{G}_{2}\right)+\beta_{1}\left(\hat{G}_{2}\right)-1\right\} \\
& =d\left(G_{1}\right)+d\left(G_{2}\right) \\
& \leq \mathbf{d}\left(\Gamma_{1}\right)+\mathbf{d}\left(\Gamma_{2}\right) .
\end{aligned}
$$

Finally, suppose that some circuit, $C_{1}$ say, splits into several pieces by $U$. $C_{1}$ is no longer a circuit of $G_{1}$. However, a part of $C_{1}$ forms at least one circuit in $\hat{G}_{2}$, and therefore $\beta_{1}(G) \leq \beta_{1}\left(G_{1}\right)+\beta_{1}\left(\hat{G}_{2}\right)$. On the other hand, (4.8) is also valid for $G$ and $G_{i}$, and hence we obtain (4.6).

A proof of Theorem 4.4 is now complete.

Remark 4.1. In (4.6), $\mathrm{d}\left(\Gamma_{1}\right)$ cannot be replaced by $\mathrm{d}\left(\Gamma_{1} / U\right)$. In other words, $\mathbf{d}(\Gamma) \leq \mathbf{d}\left(\Gamma_{1} / U\right)+\mathbf{d}\left(\Gamma_{2} / U\right)$ need not be true. However, for more details see [M6].

The following theorem will frequently be used to determine $d(\Gamma)$.

Theorem 4.6. For any connected graph $\Gamma$,

$$
\left[\frac{|E(\Gamma)|+1}{2}\right] \leq \mathbf{d}(\Gamma) \leq|E(\Gamma)| .
$$

Proof. To prove the first inequality, first we note that $\mathrm{d}(\Gamma) \geq d(G)$ for any spanning subgraph $G$. Letting $G$ be $V_{\Gamma}$ or $\Gamma$, we have

$$
\left\{\begin{array}{l}
\mathbf{d}(\Gamma) \geq d\left(V_{\Gamma}\right)=|V(\Gamma)|-1 \text { and } \\
\mathbf{d}(\Gamma) \geq d(\Gamma)=\beta_{1}(\Gamma)
\end{array}\right.
$$

Since $\Gamma$ is connected, $\beta_{0}(\Gamma)=1$, and hence it follows from (4.10) that

$$
2 \mathbf{d}(\Gamma) \geq|V(\Gamma)|-1+\beta_{1}(\Gamma)=\beta_{0}(\Gamma)-1+|E(\Gamma)|=|E(\Gamma)|,
$$

which is equivalent to the first inequality.

Now we prove the second inequality. If $|E(\Gamma)|=0$ or 1 , the inequality is obvious. Suppose inductively that $\mathbf{d}\left(\Gamma^{\prime}\right) \leq\left|E\left(\Gamma^{\prime}\right)\right|$ for any graph $\Gamma^{\prime}$ with 
$\left|E\left(\Gamma^{\prime}\right)\right|<|E(\Gamma)|$. If $\Gamma$ consists of loops and isthmuses, then Theorem 4.3 (3) shows that $\mathrm{d}(\Gamma)=|E(\Gamma)|$, and we are done. Suppose that $\Gamma$ has an edge $e$, not a loop or an isthmus. Since $\mathrm{d}(\Gamma-e) \leq|E(\Gamma-e)|=|E(\Gamma)|-1$ and $\mathrm{d}(\Gamma / e) \leq|E(\Gamma)|-1$ by the induction assumption, it follows from (4.3) that $\mathrm{d}(\Gamma) \leq|E(\Gamma)|-1 \leq|E(\Gamma)|$. This proves the second inequality. Q.E.D.

If $\mathbf{d}(\Gamma)$ has the extreme value, then we can almost determine the graph $\Gamma$.

Theorem 4.7. Let $\Gamma$ be a connected graph. Suppose $\mathrm{d}(\Gamma)=[(|E(\Gamma)|+1) / 2]$. Then, if $|E(\Gamma)|$ is even, $V_{\Gamma}$ and $\Gamma$ are maximal. If $|E(\Gamma)|$ is odd, then $V_{\Gamma}$ or $\Gamma$ (but not both) is maximal.

Proof. Suppose that $|E(\Gamma)|$ is even. Then $2 \mathrm{~d}(\Gamma)=|E(\Gamma)|$, and hence (4.10) implies that $\mathbf{d}(\Gamma)=d\left(V_{\Gamma}\right)=|V(\Gamma)|-1$ and $\mathbf{d}(\Gamma)=\beta_{1}(\Gamma)$. Therefore, both $V_{\Gamma}$ and $\Gamma$ are maximal. If $|E(\Gamma)|$ is odd, then $2 \mathrm{~d}(\Gamma)=|E(\Gamma)|+1$, and hence either $\mathbf{d}(\Gamma)=|V(\Gamma)|-1$ and $\mathbf{d}(\Gamma)=\beta_{1}(\Gamma)+1$, or $\mathbf{d}(\Gamma)=|V(\Gamma)|$ and $\mathbf{d}(\Gamma)=\beta_{1}(\Gamma)$. In the first case $V_{\Gamma}$ is maximal, while in the second case $\Gamma$ is maximal, but $V_{\Gamma}$ and $\Gamma$ cannot be maximal simultaneously.

Theorem 4.8. Let $\Gamma$ be a connected graph.

(i) $\mathrm{d}(\Gamma)=|E(\Gamma)|$ iff $\Gamma$ consists of only loops and isthmuses; i.e., $\Gamma$ is a tree with loops attached.

(ii) $\mathbf{d}(\Gamma)=|E(\Gamma)|-1$ iff $\Gamma$ consists of loops, isthmuses, and either exactly one circuit or one multiple-edge graph; i.e., $\Gamma$ is a circuit or a multipleedge graph with the graph obtained in (i) attached.

Proof. (i) The "if" part follows from Theorem 4.3 (3). To prove the converse, suppose that $\mathrm{d}(\Gamma)=|E(\Gamma)|$. If $\Gamma$ has an edge $e$, not a loop or an isthmus, then (4.3) would yield $\mathrm{d}(\Gamma) \leq|E(\Gamma)|-1$, since $\mathrm{d}(\Gamma-(e))$ and $\mathrm{d}(\Gamma / e) \leq|E(\Gamma)|-1$. It contradicts the assumption.

(ii). First we assume that $\Gamma$ is not separable. Then the "if" part is easy. Conversely, suppose $\mathrm{d}(\Gamma)=|E(\Gamma)|-1$. The case where $|E(\Gamma)|=0$ or 1 cannot occur. If $|E(\Gamma)|=2$, then $\Gamma$ is a multiple-edge graph, since $\Gamma$ is not separable, and we are done.

Now suppose inductively that (ii) holds for any (nonseparable) graph $\Gamma^{\prime}$ with $\left|E\left(\Gamma^{\prime}\right)\right|<|E(\Gamma)|$ and $\mathrm{d}\left(\Gamma^{\prime}\right)=\left|E\left(\Gamma^{\prime}\right)\right|-1$. Take an edge $e$ from $\Gamma$. Since $\Gamma$ is not separable, $e$ is neither a loop nor an isthmus. Therefore, (4.3) implies that at least one of $\mathrm{d}(\Gamma-(e))$ and $\mathrm{d}(\Gamma / e)$ must be $|E(\Gamma)|-1$.

Suppose that $\mathrm{d}(\Gamma-(e))=|E(\Gamma)|-1=|E(\Gamma-(e))|$. Then (i) implies that $\Gamma-(e)$ must be a tree with loops attached. Since $\Gamma$ has no loops and isthmuses, $\Gamma-(e)$ must be a tree with only two vertices of valency one, and these vertices are ends of $e$. Therefore, $\Gamma$ is a circuit. On the other hand, if $\mathrm{d}(\Gamma / e)=|E(\Gamma)|-1=|E(\Gamma / e)|$, then $\Gamma / e$ must be one-vertex graph to which many loops are attached, since $\Gamma$ is not separable. Therefore, $\Gamma$ is a multiple-edge graph. This proves (ii) for a nonseparable graph.

Now suppose that $\Gamma$ is separable. Then (4.2) and (4.9) show that only one block, $\Gamma_{1}$, say, has the chromatic degree $\left|E\left(\Gamma_{1}\right)\right|-1$, and other blocks $\Gamma_{i}$ 
$(i \neq 1)$ have chromatic degree $\left|E\left(\Gamma_{i}\right)\right|$. Then apply the previous argument and (i), Q.E.D.

Corollary 4.9. If $\Gamma$ is not separable, and $|E(\Gamma)| \geq 1$, then

$$
\left[\frac{|E(\Gamma)|+1}{2}\right] \leq \mathbf{d}(\Gamma) \leq|E(\Gamma)|-1
$$

Furthermore, $\mathbf{d}(\Gamma)=|E(\Gamma)|-1$ iff $\Gamma$ is either a circuit or a multiple-edge graph.

\section{MAXIMAL SPANNING SUBGRAPHS}

In this section, we will study the properties of maximal spanning subgraphs of $\Gamma$ and will determine maximal subgraphs of certain graphs, including the complete graph. First we may assume without loss of generality that $\Gamma$ has no loops, because if $\Gamma$ has loops, any maximal spanning subgraph must contain them.

Proposition 5.1. Suppose that $\Gamma$ contains multiple edges $e_{1}, \ldots, e_{m}$ having the same ends $a$ and $b$, say.

If $m \geq 3$, then any maximal spanning subgraph must contain these multiple edges.

When $m=2$, some maximal spanning subgraph $G$ may not have these edges $e_{1}, e_{2}$, but $G^{\prime}=G \cup\left\{e_{1}, e_{2}\right\}$ is also a maximal spanning subgraph of $\Gamma$.

Proof. Let $G$ be a maximal spanning subgraph of $\Gamma$.

Case (I). $a$ and $b$ belong to different connected components of $G$. Then $G^{\prime}=G \cup\left\{e_{1}, \ldots, e_{m}\right\}$ is also a spanning subgraph, and

$$
G^{\prime} \in \mathscr{S}_{\Gamma}\left(r_{G}-1, s_{G}+m-1\right)
$$

and hence $d\left(G^{\prime}\right)=r_{G}+s_{G}+m-2$. If $m \geq 3$, then $d\left(G^{\prime}\right) \supsetneqq d(G)$, a contradiction. If $m=2$, then $d\left(G^{\prime}\right)=d(G)$ and $G^{\prime}$ is also maximal.

Case (II). $a$ and $b$ belong to the same component of $G$. Let $G^{\prime}=G \cup$ $\left\{e_{1}, \ldots, e_{m}\right\}$. Then $G^{\prime} \in \mathscr{S}_{\Gamma}\left(r_{G}, s_{G}+k\right)$, where $k$ is the number of those edges $e_{1}, \ldots, e_{m}$ which are not in $G$. If $k \geq 1$, then $d\left(G^{\prime}\right)>d(G)$, a contradiction and hence $G=G^{\prime}$. Q.E.D.

A connected subgraph $H$ of $\Gamma$ is called an m-chain if (1) $V(H)=\left\{v_{1}, \ldots\right.$, $\left.v_{m+1}\right\}$, where $v_{i} \neq v_{j}$ if $i \neq j$, and (2) $E(H)=\left\{e_{1}, \ldots, e_{m}\right\} ;(3) v_{i}$ and $v_{i+1}$ are ends of $e_{i}, i=1,2, \ldots, m$. Furthermore, if all vertices, except the first $v_{1}$ and last $v_{m+1}$, have valency 2 in $\Gamma$, an $m$-chain is said to be pure.

The following proposition is the dual to Proposition 5.1, and therefore a proof will be omitted.

Proposition 5.2. Suppose that $\Gamma$ has a pure m-chain $H$. If $m \geq 3$, any maximal spanning subgraph cannot contain $m$ edges $e_{1}, \ldots, e_{m}$. If $m=2$, 
there may exist a maximal spanning subgraph $G$ that contains $e_{1}$ and $e_{2}$, but $G-\left\{e_{1}, e_{2}\right\}$ is also a maximal spanning subgraph of $\Gamma$.

The following proposition is quite useful in finding maximal spanning subgraphs.

Proposition 5.3. Let $G$ be a maximal spanning subgraph of $\Gamma$. Suppose that $G$ contains an edge $e$. Then $G /(e)$ is also maximal in $\Gamma /(e)$. Furthermore, if $G \in \mathscr{S}_{\Gamma}(r, s)$, then $G /(e) \in \mathscr{S}_{\Gamma /(e)}(r, s)$, and hence $d(G)=d(G /(e))$.

More generally, if $G$ contains multiple edges $e_{1}, \ldots, e_{m}$, then $G_{*}=$ $\left(G-\left\{e_{1}, \ldots, e_{m-1}\right\}\right) /\left(e_{m}\right)$ is also maximal in $\Gamma_{*}=\left(\Gamma-\left\{e_{1}, \ldots, e_{m-1}\right\}\right) /\left(e_{m}\right)$, and $d(G)=d\left(G_{*}\right)+m-1$.

Proof. First, $e$ is not an isthmus. In fact, if $e$ is an isthmus, then $G^{\prime}=$ $G-(e)$ belongs to $\Gamma\left(r_{G}+1, s_{G}\right)$ and hence $d\left(G^{\prime}\right)=r_{G}+s_{G}+1>d(G)$, which contradicts the maximality of $G$. Theorem 4.3(5) now implies that $\mathrm{d}(G)=$ $\max \{\mathbf{d}(\Gamma-(e)), \mathbf{d}(\Gamma /(e))\}$. However, it is clear that $\beta_{0}(G-(e))=\beta_{0}(G)$ and $\beta_{1}(G-(e))=\beta_{1}(G)-1$, while $\beta_{0}(G /(e))=\beta_{0}(G)$ and, since $e$ is not a loop, $\beta_{1}(G /(e))=\beta_{1}(G)$. Therefore, $d(G /(e))=d(G) \geq \mathrm{d}(\Gamma /(e))$, and hence $G /(e)$ is maximal in $\Gamma /(e)$.

The general case is proved in a similar manner. Note that

$$
G_{*} \in \mathscr{S}_{\Gamma_{*}}\left(r_{G}, s_{G}-m+1\right) . \quad \text { Q.E.D. }
$$

Using these propositions, we can determine $\mathbf{d}(\Gamma)$ for some simple graphs.

Example 5.1. If $\Gamma$ is an $n$-circuit, then $\mathbf{d}(\Gamma)=n-1$, and $V_{\Gamma}$ is a maximal spanning subgraph.

Example 5.2. If $\Gamma$ is an $n$-wheel, then $\mathbf{d}(\Gamma)=n$, and $V_{\Gamma}$ and $\Gamma$ are maximal spanning subgraphs. (See Figure 5.1.)

In fact, if a maximal spanning subgraph $G$ has an edge, $e_{1}$ say, Proposition 5.3 shows that $G_{1}=G /\left(e_{1}\right)$ is also maximal in $\Gamma /\left(e_{1}\right)=\Gamma_{1}$. However, $\Gamma_{1}$ now contains multiple edges $e_{1}^{\prime}, e_{2}^{\prime}$, and hence we may assume that $G_{1}$ contains $e_{1}^{\prime}$ and $e_{2}^{\prime}$. Then Proposition 5.3 is applied again to show that $\left(G_{1}-\left(e_{1}^{\prime}\right)\right) /\left(e_{2}^{\prime}\right)=G_{2}$ is maximal in $\left(\Gamma_{1}-\left(e_{1}^{\prime}\right)\right) /\left(e_{2}^{\prime}\right)=\Gamma_{2} . \Gamma_{2}$ now contains multiple edges. Repeat the same argument so that eventually $\Gamma_{q}$ becomes a single vertex. Therefore the original maximal spanning subgraph $G$ must be $\Gamma$. Computations of $d(\Gamma)$ and $d\left(V_{\Gamma}\right)$ conclude that $\mathbf{d}(\Gamma)=n$.

A similar argument will prove the following.

Proposition 5.4. Let $\Gamma_{m}$ be the complete m-graphs. If $m=3$, a maximal spanning subgraph is the spanning vertex graph. If $m \geq 4$, a maximal spanning subgraph is $\Gamma$ itself. Therefore, $\mathbf{d}\left(\Gamma_{3}\right)=2$ and $\mathbf{d}\left(\Gamma_{m}\right)=(m-1)(m-2) / 2$ for $m \geq 4$.

Proof. Since $\beta_{0}\left(\Gamma_{m}\right)-\beta_{1}\left(\Gamma_{m}\right)=\left|V\left(\Gamma_{m}\right)\right|-\left|E\left(\Gamma_{m}\right)\right|$, it follows that $\beta_{1}\left(\Gamma_{m}\right)=$ $1-m+\left(\begin{array}{c}m \\ 2\end{array}\right)=\left(\begin{array}{c}m-1 \\ 2\end{array}\right)$, and hence $\mathbf{d}\left(\Gamma_{m}\right)=0+\beta_{1}\left(\Gamma_{m}\right)=\left(\begin{array}{c}m-1 \\ 2\end{array}\right)$. 


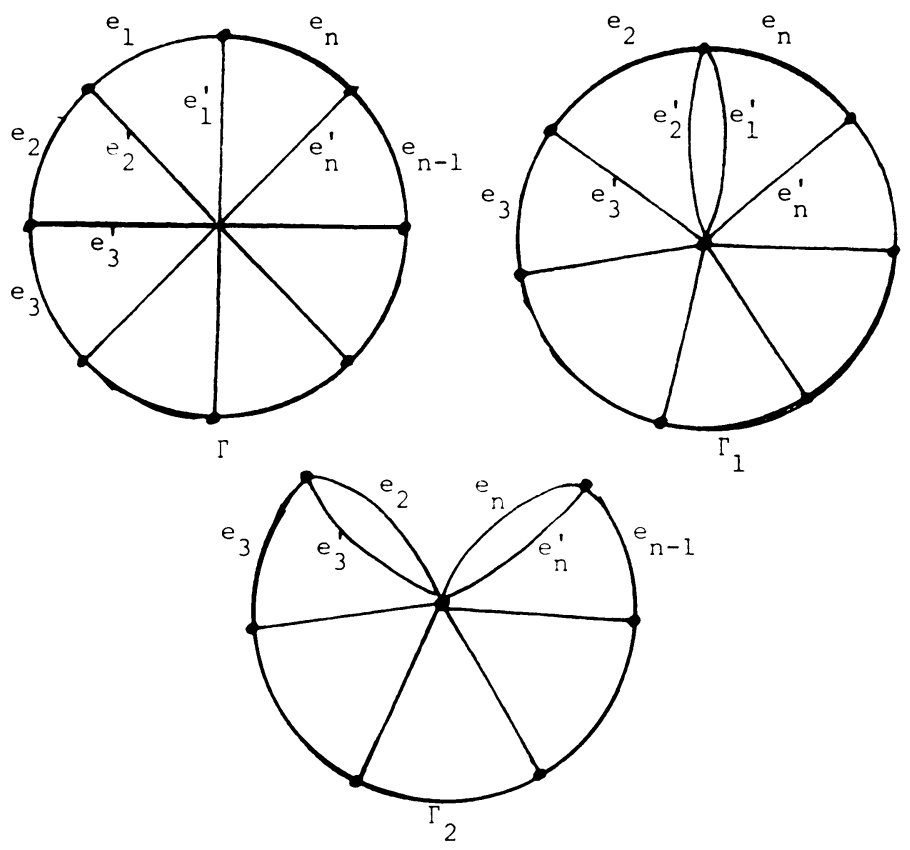

FIGURE 5.1

Proposition 5.5. Let $\Gamma$ be a triangulation of $S^{2}$. Suppose $|V(\Gamma)| \geq 4$. Then $\Gamma$ itself is a maximal spanning subgraph of $\Gamma$, and hence $\mathbf{d}(\Gamma)=\beta_{1}(\Gamma)$.

Proof. By the same argument as used before, we can easily prove that if a maximal spanning subgraph $G$ contains an edge, then $G=\Gamma$. Now we compute $d(\Gamma)$ and $d\left(V_{\Gamma}\right)$.

Obviously, $d(V(\Gamma))=|V(\Gamma)|-1$ and $d(\Gamma)=d-1$, where $d$ is the number of domains in $S^{2}-\Gamma$. Since $\Gamma$ is a triangulation of $S^{2}$, it follows that $2|E(\Gamma)|=$ $3 d$, while $|V(\Gamma)|-|E(\Gamma)|+d=2$, since $\Gamma$ is a planar graph. Combining these equalities, we obtain that $3 d=2(|V(\Gamma)|+d-2)$. Since $|V(\Gamma)| \geq 4$, it follows that $d-1=2|V(\Gamma)|-5 \geq|V(\Gamma)|-1$. This proves Proposition 5.5.

Examples 5.1 and 5.2 and these propositions may mislead the reader into believing that a maximal spanning subgraph is either the spanning vertex graph

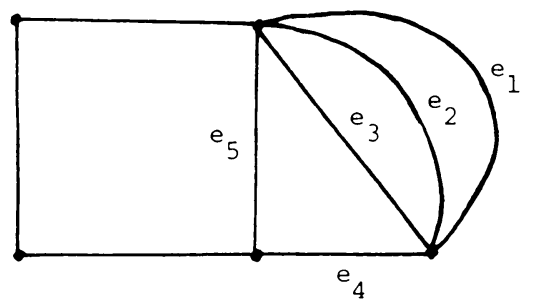

FIGURE 5.2 
or $\Gamma$ itself. This is not always the case, however, as is shown in the following simple graph $\Gamma$ (in Figure 5.2).

A maximal spanning subgraph $G$ of $\Gamma$ consists of five edges $e_{1}, e_{2}, \ldots, e_{5}$ and all vertices. Then $G \in \mathscr{S}_{\Gamma}(2,3)$ and hence $d(G)=5$, but $\Gamma \in \mathscr{S}_{\Gamma}(0,4)$ and $V(\Gamma) \in \mathscr{S}_{\Gamma}(4,0)$. In fact, $\mathrm{d}(\Gamma)=5$.

\section{COEFFICIENTS OF POLYNOMIALS}

By definition, $\mathscr{S}_{\Gamma}(0,0)$ is the set of spanning trees of $\Gamma$. It will be seen in $\S 7$ that $\left|\mathscr{S}_{\Gamma}(0,0)\right|$ can be calculated by means of the adjacency matrix of $\Gamma$ (Proposition 7.2). Besides $\left|\mathscr{S}_{\Gamma}(0,0)\right|$, the explicit evaluation of $\left|\mathscr{S}_{\Gamma}(r, s)\right|$ seems extremely difficult, even for a positive graph. In this section, we will determine some of these $\left|\mathscr{S}_{\Gamma}(r, s)\right|$ with large $r$ or $s$. The results will be used in the later sections.

Proposition 6.1. Let $\Gamma$ be a connected positive graph. Let $v=|V(\Gamma)|$ and $t=\beta_{1}(\Gamma)$. Then

(1)(i) $\left|\mathscr{S}_{\Gamma}(v-1,0)\right|=1$.

(ii) If $\Gamma$ has $k$ loops, then for $i \geq 1,\left|\mathscr{S}_{\Gamma}(v-1, i)\right|=\left(\begin{array}{c}k \\ i\end{array}\right)$.

(2)(i) $\left|\mathscr{S}_{\Gamma}(0, t)\right|=1$.

(ii) If $\Gamma$ has $l$ isthmuses, then for $j \geq 1,\left|\mathscr{S}_{\Gamma}(j, t)\right|=\left(\begin{array}{l}l \\ j\end{array}\right)$.

Proof. Let $G \in \mathscr{S}_{\Gamma}\left(r_{G}, s_{G}\right)$. Since $r_{G}-s_{G}+1$ is equal to Euler characteristic of $G$, we see that

$$
r_{G}-s_{G}=v-1-|E(G)| \text {. }
$$

Proof of (1). Let $G \in \mathscr{S}_{\Gamma}(v-1, i), i \geq 0$. Then (6.1) shows that $|E(G)|=i$, and hence $\mathscr{S}_{\Gamma}(v-1,0)$ consists of only the spanning vertex graph $V_{\Gamma}$, and $\mathscr{S}_{\Gamma}(v-1,0) \mid=1$.

Suppose $i=|E(G)| \geq 1$. Let $e_{1}, e_{2}, \ldots, e_{k}$ be all loops in $\Gamma$. Consider $\Gamma_{0}=\Gamma-\left\{e_{1}, e_{2}, \ldots, e_{k}\right\}$. Suppose $E(G) \cap E\left(\Gamma_{0}\right) \neq \varnothing$. Since $\Gamma_{0}$ has no loops, every edge in $\Gamma_{0}$ has two ends, and hence $\beta_{0}(G) \lessgtr v$, a contradiction. Therefore, $E(G) \cap E\left(\Gamma_{0}\right)=\varnothing$, and every edge of $G$ is a loop. Since there are exactly $k$ loops in $\Gamma$, we have $\mathscr{S}_{\Gamma}(v-1, i) \mid=\left(\begin{array}{c}k \\ i\end{array}\right)$.

Proof of (2). Let $G \in \mathscr{S}_{\Gamma}(j, t)$. Then $|E(G)|=v-1-(j-t)=|E(\Gamma)|-j$, since $|E(\Gamma)|=v-1+t$. If $j=0$, then $G=\Gamma$ and $\mathscr{S}_{\Gamma}(0, t) \mid=1$.

Suppose $j=|E(\Gamma)|-|E(G)| \geq 1$. Let $e_{1}, e_{2}, \ldots, e_{l}$ be all isthmuses in $\Gamma$, and consider $\Gamma_{0}=\Gamma-\left\{e_{1}, e_{2}, \ldots, e_{l}\right\}$. Suppose $E\left(G \cap \Gamma_{0}\right) \neq E\left(\Gamma_{0}\right)$. Since $\Gamma_{0}$ has no isthmuses, it follows that for every edge $e$ in $\Gamma_{0}, \Gamma_{0}-(e)$ is connected, and hence $\beta_{1}\left(\Gamma_{0}-(e)\right)=\beta_{1}\left(\Gamma_{0}\right)-1=t-1$ and $\beta_{1}(G) \supsetneqq t$, a contradiction. Therefore, $E\left(G \cap \Gamma_{0}\right)=E\left(\Gamma_{0}\right)$. Then, since $|E(\Gamma)|-|E(G)|=j, G$ is obtained from $\Gamma$ by deleting $j$ isthmuses, and hence $\left|\mathscr{S}_{\Gamma}(j, t)\right|=\left(\begin{array}{l}l \\ j\end{array}\right)$. Q.E.D.

\section{Proposition 6.2.}

(1)(i) If $\Gamma$ contains $k$ loops, then $\left|\mathscr{S}_{\Gamma}(v-2,0)\right|=|E(\Gamma)|-k$.

(ii) If $\Gamma$ is simple, then for any $i \geq 1,\left|\mathscr{S}_{\Gamma}(v-2, i)\right|=0$. 
(2)(i) If $\Gamma$ contains $l$ isthmuses, then $\left|\mathscr{S}_{\Gamma}(0, t-1)\right|=|E(\Gamma)|-l$.

(ii) If $\Gamma$ has neither isthmuses nor twigs, then for any $j \geq 1$, $\left|\mathscr{S}_{\Gamma}(j, t-1)\right|=0$, where a twig is a vertex with valency 2 .

Proof. (1) Let $G \in \mathscr{S}_{\Gamma}(v-2, i)$. Then $|E(G)|=v-1-(v-2-i)=i+1$.

(i) Suppose $i=0$. Then $G$ has only one edge $e . e$ is not a loop, otherwise $\beta_{1}(G) \neq 0$. Therefore, there are only $|E(\Gamma)|-k$ spanning subgraphs $G$ with one edge and $\beta_{1}(G)=0$.

(ii) Suppose $i \geq 1$. Since $\Gamma$ is simple, any two distinct edges are incident to at least three distinct vertices. Therefore, $\beta_{0}(G)-1 \leq v-3$, and hence $\mathscr{S}_{\Gamma}(v-2, i)=\varnothing$ for $i \geq 1$.

A proof of (2) is analogous and hence is omitted.

Finally, we will prove the following unexpected theorem.

Theorem 6.3. Let $\mathscr{S}_{\Gamma}\left(r_{1}, s_{1}\right), \mathscr{S}_{\Gamma}\left(r_{2}, s_{2}\right), \ldots, \mathscr{S}_{\Gamma}\left(r_{k}, s_{k}\right)$ be a sequence of all nonempty sets of maximal spanning subgraphs of $\Gamma$ such that $r_{i}+s_{i}=\mathbf{d}(\Gamma)$, $i=1,2, \ldots, k$ and $r_{1}<r_{2}<\cdots<r_{k}$. Then

$$
\left|\mathscr{S}_{\Gamma}\left(r_{1}, s_{1}\right)\right|=1 \text { and }\left|\mathscr{S}_{\Gamma}\left(r_{k}, s_{k}\right)\right|=1 \text {. }
$$

Proof. For convenience, we call $\mathscr{S}_{\Gamma}\left(r_{1}, s_{1}\right)$ and $\mathscr{S}_{\Gamma}\left(r_{k}, s_{k}\right)$ the first and the last sets of maximal spanning subgraphs of $\Gamma$, respectively. We prove only that $\left|\mathscr{S}_{\Gamma}\left(r_{1}, s_{1}\right)\right|=1$, since a proof of another formula is completely analogous.

Now a proof will be given by induction on $|E(\Gamma)|$. We may assume without loss of generality that $\Gamma$ is connected and is not separable. (See Theorem 4.3.) Since the theorem is trivially true for $|E(\Gamma)|=0$ or 1 , we assume inductively that the theorem holds for any graph $\Gamma^{\prime}$ with $\left|E\left(\Gamma^{\prime}\right)\right|<|E(\Gamma)|$. Take $G_{1}$ and $G_{2}$ from $\mathscr{S}_{\Gamma}\left(r_{1}, s_{1}\right)$.

Case 1. $E\left(G_{1}\right) \cap E\left(G_{2}\right) \neq \varnothing$. Let $e \in E\left(G_{1}\right) \cap E\left(G_{2}\right)$. Then $G_{1} /(e)=G_{1}^{\prime}$ and $G_{2} /(e)=G_{2}^{\prime}$ are maximal spanning subgraphs of $\Gamma /(e)=\Gamma^{\prime}$ (Proposition 5.3). Note that $G_{1}^{\prime}, G_{2}^{\prime} \in \mathscr{S}_{\Gamma^{\prime}}\left(r_{1}, s_{1}\right)$. Furthermore, it is easy to see that $\mathscr{S}_{\Gamma^{\prime}}\left(r^{\prime}, s^{\prime}\right)$ is the first set of maximal spanning subgraphs of $\Gamma^{\prime}$. Therefore the induction assumption implies that $G_{1}^{\prime}=G_{2}^{\prime}$ in $\Gamma$ and hence $G_{1}=G_{2}$.

Case 2. $E\left(G_{1}\right) \cap E\left(G_{2}\right)=\varnothing$. Suppose that there is an edge $e$ of $\Gamma$, not in $G_{1}$ or $G_{2}$. Then $G_{1}^{\prime}=G_{1}-(e)$ and $G_{2}^{\prime}=G_{2}-(e)$ are maximal spanning subgraphs in $\Gamma^{\prime}=\Gamma-(e)$. Since $\mathscr{S}_{\Gamma^{\prime}}\left(r_{1}, s_{1}\right) \quad\left(\ni G_{1}^{\prime}, G_{2}^{\prime}\right)$ is the first set of maximal spanning subgraphs of $\Gamma^{\prime}$, it follows from the induction assumption that $G_{1}^{\prime}=G_{2}^{\prime}$ and hence $G_{1}=G_{2}$, as required.

Therefore we may assume finally that each edge in $\Gamma$ belongs to either $G_{1}$ or $G_{2}$, but not both. Since $G_{1}$ and $G_{2}$ are spanning subgraphs, $G_{1}$ is the spanning complement of $G_{2}$ in $\Gamma$. Therefore, it follows from Theorem 3.4 that

$$
r_{G_{1}}+s_{G_{1}}+r_{G_{2}}+s_{G_{2}} \leq|E(\Gamma)| \text {. }
$$


Since $r=r_{G_{1}}=r_{G_{2}}, s=s_{G_{1}}=s_{G_{2}}$, and $r+s=\mathrm{d}(\Gamma)$, we have from (6.2) that $2 \mathrm{~d}(\Gamma)=2(r+s) \leq|E(\Gamma)|$, and hence

$$
\mathrm{d}(\Gamma) \leq[|E(\Gamma)| / 2] .
$$

On the other hand, Corollary 4.9 shows that

$$
[(E(\Gamma)+1) / 2] \leq \mathbf{d}(\Gamma) .
$$

Combining (6.3) and (6.4), we see that $|E(\Gamma)|$ is even and $\mathrm{d}(\Gamma)=|E(\Gamma)| / 2$. Therefore, we must have equality in (6.2) (or (3.7)). Since $\Gamma$ is not separable, it follows from Theorem 3.4 that $G_{1}=\Gamma$ and $G_{2}=V_{\Gamma}$ (or $G_{1}=V_{\Gamma}$ and $G_{2}=\Gamma$ ). Then $G_{1} \in \mathscr{S}_{\Gamma}\left(0, \beta_{1}(\Gamma)\right)$ and $G_{2} \in \mathscr{S}_{\Gamma}(|V(\Gamma)|-1,0)$ (or $G_{1} \in \mathscr{S}_{\Gamma}(|V(\Gamma)|-1,0)$ and $G_{2} \in \mathscr{S}_{\Gamma}\left(0, \beta_{1}(\Gamma)\right)$. This is possible only if $|V(\Gamma)|-1=0$ and $\beta_{1}(\Gamma)=0$, i.e., $\Gamma$ is the one-vertex graph, and then $G_{1}=G_{2}=\Gamma$. This proves Theorem 6.3.

\section{SigNATURE OF A GRAPH}

In this section, we introduce another invariant of a weighted graph, and in the next section we will prove the second main theorem (Theorem 8.1).

Definition 7.1. Let $\Gamma$ be a (weighted) graph. The adjacency matrix of $\Gamma$ is a $|V(\Gamma)| \times|V(\Gamma)|$ integer matrix $A_{\Gamma}=\left\|a_{i j}\right\|$ defined as follows.

(7.1)(1) For any $i \neq j, a_{i j}$ is the number of those positive edges minus negative edges which have two ends $v_{i}$ and $v_{j}$.

(2) $a_{i i}=0$ for $i=1,2, \ldots,|V(\Gamma)|$.

Let $D_{\Gamma}=\left\|d_{i j}\right\|$ be a diagonal matrix of order $|V(\Gamma)|$, where $d_{i i}=\sum_{j=1}^{|V(\Gamma)|} a_{i j}$, $i=1,2, \ldots,|V(\Gamma)|$. Then the matrix $B_{\Gamma}=D_{\Gamma}-A_{\Gamma}$ is called the matrix of $\Gamma$.

We should note that if $\Gamma$ is positive then $B_{\Gamma}$ coincides with the known matrix of a (unweighted) graph considered in [Bi, §4]. $B_{\Gamma}$ is not affected by the presence of loops in $\Gamma$.

$B_{\Gamma}$ is also defined using the incidence matrix $C_{\Gamma}$ of $\Gamma . C_{\Gamma}=\left\|c_{i j}\right\|$ is a $|V(\Gamma)| \times|E(\Gamma)|$ integer matrix defined in a usual way for $\Gamma$ (without a weight). Each row corresponds to a vertex and each column corresponds to an edge. If an $i$ th edge $e_{i}$ is a loop, then the $i$ th column of $C_{\Gamma}$ is 0 . However, if $e_{i}$ is not a loop and has two ends $v_{k}$ and $v_{l}$, then the $i$ th column has exactly two nonzero entries $c_{k i}=1$ and $c_{l, i}=-1$ (or $c_{k i}=-1$ and $c_{l i}=1$ ).

Let $F_{\Gamma}=\left\|f_{i j}\right\|$ be a diagonal matrix of order $|E(\Gamma)|$, where $f_{i i}$ is the weight of $e_{i}$.

Proposition 7.1. $B_{\Gamma}=C_{\Gamma} F_{\Gamma} C_{\Gamma}^{t}$, where $C_{\Gamma}^{t}$ denotes the transpose of $C_{\Gamma}$.

Proposition 7.2. The determinant of $\Gamma$, denoted by $\operatorname{det} \Gamma$, is defined as a principal minor of $B_{\Gamma}$ of order $|V(\Gamma)|-1$. Then det $\Gamma$ gives the number of (weighted) spanning trees in $\Gamma$ or, more precisely,

$$
\operatorname{det} \Gamma=\sum_{G \in \mathcal{S}_{\Gamma}(0,0)}(-1)^{n(G)}=\sum_{T \subset \Gamma e_{i} \in E(\Gamma)} \prod_{\Gamma} f_{\Gamma}\left(e_{i}\right),
$$

where the second summation runs over all spanning trees $T$ in $\Gamma$. 
Proofs of Propositions 7.1 and 7.2 are slight modifications of those of the corresponding well-known theorems for a positive (or unweighted) graph, and we omit the details. See [BC].

Now $B_{\Gamma}$ is symmetric and singular. Each row and column of $B_{\Gamma}$ correspond to a vertex of $\Gamma$. For convenience, we call the row (or column) of $B_{\Gamma}$ corresponding to a vertex $v_{0}$ the $v_{0}$-row (or $v_{0}$-column).

Let $B_{\Gamma}\left(i_{1}, i_{2}, \ldots, i_{k}\right)$ denote the principal submatrix of $B_{\Gamma}$ which does not involve $i_{1}$ th- $i_{2}$ th $\cdots i_{k}$ th-rows and columns. Sometimes we write $B_{\Gamma}\left(v_{l_{1}}, \ldots, v_{l_{k}}\right)$ instead of $B_{\Gamma}\left(i_{1}, \ldots, i_{k}\right)$ if the $i_{j}$ th row corresponds to the vertex $v_{l,}$.

Definition 7.2. The signature of $B_{\Gamma}$ is called the signature of $\Gamma$ and is denoted by $\sigma(\Gamma)$.

Since $B_{\Gamma}$ is singular, the signature of $B_{\Gamma}$ coincides with the signature of $B_{\Gamma}(i)$ for any $i, 1 \leq i \leq|V(\Gamma)|$, and $|\sigma(\Gamma)| \leq|V(\Gamma)|-1$.

\section{Proposition 7.3.}

(1) $\sigma(\Gamma)$ is an invariant of a graph.

(2) If $\hat{\Gamma}$ is the opposite graph of $\Gamma$, then $\sigma(\hat{\Gamma})=-\sigma(\Gamma)$.

(3) If $\Gamma$ is a connected positive graph, then $\sigma(\Gamma)=|V(\Gamma)|-1$.

Proof. (1) and (2) follow from the definition.

(3) If $\Gamma$ is a connected positive graph, then $B_{\Gamma}(i)$ has the following properties.

(7.3) (1) All diagonal entries are positive.

(2) Nondiagonal entries are nonpositive.

(3) Each row-sum (i.e., the sum of all entries of each row) is nonnegative.

(4) Every principal submatrix of $B_{\Gamma}(i)$ has at least one row that has nonzero row-sum.

Then it is shown that $B_{\Gamma}(i)$ is positive definite. For a proof, for example, see [M1]. Thus, $\sigma(\Gamma)=|V(\Gamma)|-1$. Q.E.D.

Remark 7.1. The addition or removal of any number of loops does not change the matrix of $\Gamma$ and hence the signature of $\Gamma$.

In view of Proposition 7.3(3), $\sigma(\Gamma)$ is only interested in a "mixed" graph. Before we prove elementary properties of $\sigma(\Gamma)$, we define a few concepts.

A symmetric matrix $M$ is called completed if every row sum (and hence every column sum) is 0 . Every symmetric matrix $M$ can be extended to a complete matrix $\hat{M}$ with an extra row and column. $\hat{M}$ is called the completion of $M$.

\section{Proposition 7.4.}

(1) Let $\Gamma_{1}, \ldots, \Gamma_{m}$ be connected components of $\Gamma$. Then

(i) $\sigma(\Gamma)=\sum_{i=1}^{m} \sigma\left(\Gamma_{i}\right)$, and

(ii) $|\sigma(\Gamma)| \leq|V(\Gamma)|-m$. 
Equality holds in (ii) if all $\Gamma_{i}$ are positive or all negative.

(2) Let $\Gamma$ be a connected graph and $\Gamma_{1}, \ldots, \Gamma_{k}$ be blocks of $\Gamma$. Then

$$
\sigma(\Gamma)=\sum_{i=1}^{k} \sigma\left(\Gamma_{i}\right)
$$

(3) If $\Gamma$ is a tree with $p(\Gamma)$ positive and $n(\Gamma)$ negative edges, then

$$
\sigma(\Gamma)=p(\Gamma)-n(\Gamma) .
$$

Proof. (1) From the definition, we see that $B_{\Gamma}$ is a direct sum of $B_{\Gamma_{i}}$ and hence $\sigma\left(\Gamma_{i}\right)=\sum_{i=1}^{m} \sigma\left(\Gamma_{i}\right)$. Since $\left|\sigma\left(\Gamma_{i}\right)\right| \leq\left|V\left(\Gamma_{i}\right)\right|-1$, (ii) follows immediately. Furthermore, equality holds in (ii) iff $\sigma\left(\Gamma_{i}\right)=\left|V\left(\Gamma_{i}\right)\right|-1$ for all $i$, or $\sigma\left(\Gamma_{i}\right)=$ $-\left(\left|V\left(\Gamma_{i}\right)\right|-1\right)$ for all $i$. Therefore, if $\Gamma_{i}$ are all positive or all negative, then equality holds in (ii).

(2) Suppose that $v_{0}$ is a cut vertex of $\Gamma$, and $\Gamma=\Gamma_{1} \cup \Gamma_{2}$ and $\Gamma_{1} \cap \Gamma_{2}=$ $\left\{v_{0}\right\}$. Then $B_{\Gamma}\left(v_{0}\right)$ is a direct sum of $B_{\Gamma_{1}}\left(v_{0}\right)$ and $B_{\Gamma_{2}}\left(v_{0}\right)$, and hence $\sigma(\Gamma)=$ $\sigma\left(\Gamma_{1}\right)+\sigma\left(\Gamma_{2}\right)$. Suppose that $\Gamma_{1}$ has a cut vertex $v_{1}$ and let $\Gamma_{1}=\Gamma_{11} \cup \Gamma_{12}$ and $\Gamma_{11} \cap \Gamma_{12}=\left\{v_{1}\right\}$. Consider the completion $\hat{B}_{\Gamma_{1}}$ of $B_{\Gamma_{1}}\left(v_{0}\right)$. Since $\Gamma_{1}$ is a union of $\Gamma_{11}$ and $\Gamma_{12}$, there is a row corresponding to $v_{1}$ (which is the extended row in $\hat{B}_{\Gamma}$ if $\left.v_{0}=v_{1}\right)$ such that $\hat{B}_{\Gamma_{1}}\left(v_{1}\right)$ is the direct sum of $B_{\Gamma_{11}}\left(v_{1}\right)$ and $B_{\Gamma_{12}}\left(v_{1}\right)$. Therefore, $\sigma\left(\Gamma_{1}\right)=\sigma\left(\Gamma_{11}\right)+\sigma\left(\Gamma_{12}\right)$. Now an easy induction gives a proof of (2).

Finally, (3) follows from the fact that $\sigma(\Gamma)=1$ or -1 according as $\Gamma$ consists of one positive or negative edge, not a loop, and its ends.

Remark 7.2. The signature is an invariant for 2 -isomorphic graphs. For a proof, see [M7].

\section{SeCOND MAIN THEOREM}

Let $\Gamma$ be a weighted graph and let $P$ and $N$ be, respectively, the maximal positive and negative spanning subgraphs of $\Gamma$. The purpose of this section is to prove the following

Theorem 8.1 (Second Main Theorem). Suppose that $\Gamma$ is connected. Then

$$
r_{N}+s_{N}-n(\Gamma) \leq \sigma(\Gamma) \leq p(\Gamma)-\left(r_{P}+s_{P}\right) .
$$

Equalities hold in (8.1) simultaneously iff each block of $\Gamma$ is either a positive or negative graph.

Proof. The second statement follows from Theorem 3.4. In fact, both equalities hold simultaneously iff $r_{N}+s_{N}-n(\Gamma)=p(\Gamma)-\left(r_{P}+s_{P}\right)$ and hence $r_{N}+s_{N}+$ $r_{P}+s_{P}=p(\Gamma)+N(\Gamma)=|E(\Gamma)|$. Since $P$ and $N$ are spanning complements, Theorem 3.4 implies that $P$ (and also $N$ ) is a union of blocks of $\Gamma$, and hence each block of $\Gamma$ must be either totally positive or negative. This proves the second statement. 
Now we will prove (8.1). Since $r_{N}-s_{N}=\beta_{0}(N)-1-\beta_{1}(N)=|V(N)|-$ $|E(N)|-1=|V(\Gamma)|-n(\Gamma)-1$, we have $s_{N}=r_{N}-|V(\Gamma)|+n(\Gamma)+1$, and hence $r_{N}+s_{N}-n(\Gamma)=2 r_{N}-(|V(\Gamma)|-1)$. Similarly, since $s_{P}=r_{P}-|V(\Gamma)|+p(\Gamma)+1$, we have $p(\Gamma)-\left(r_{P}+s_{P}\right)=(|V(\Gamma)|-1)-2 r_{P}$. Therefore, (8.1) is equivalent to

$$
2 r_{N}-|V(\Gamma)|+1 \leq \sigma(\Gamma) \leq|V(\Gamma)|-1-2 r_{P} .
$$

To prove (8.2), we need the following lemma.

Lemma 8.2. Let $\Gamma$ be a connected graph. Let $\Gamma_{+}\left(\right.$or $\left.\Gamma_{-}\right)$be the graph obtained from $\Gamma$ by adding finitely many, $h$ say, positive (or negative) edges, each of which has the same ends, $a$ and $b$, say. Then

$$
\begin{array}{ll}
\text { (1) } & \sigma(\Gamma) \leq \sigma\left(\Gamma_{+}\right) \leq \sigma(\Gamma)+2 \text {, and } \\
\text { (2) } \sigma(\Gamma)-2 \leq \sigma\left(\Gamma_{-}\right) \leq \sigma(\Gamma) \text {. }
\end{array}
$$

Proof. We prove only $(8.3)(1)$, since a proof of (2) is completely analogous.

To compute $\sigma(\Gamma)$ and $\sigma\left(\Gamma_{+}\right)$, consider the matrices $B_{\Gamma}$ and $B_{\Gamma_{+}}$. For simplicity, we assume that vertices $a$ and $b$ correspond, respectively to, the first and second rows (and columns) of $B_{\Gamma}$ and $B_{\Gamma_{+}}$.

Now from the definition it is easy to see that $B_{\Gamma}$ and $B_{\Gamma_{+}}$are identical except for the top left $2 \times 2$ principal submatrices, which are of the form

$$
\left[\begin{array}{ll}
a_{11} & a_{12} \\
a_{21} & a_{22}
\end{array}\right] \text { and }\left[\begin{array}{ll}
a_{11}+h & a_{12}-h \\
a_{21}-h & a_{22}+h
\end{array}\right] \text {, }
$$

respectively. Let $M=B_{\Gamma}(a b)=B_{\Gamma_{+}}(a b)$. Since $M$ is symmetric, there is a $(|V(\Gamma)|-2) \times(|V(\Gamma)|-2)$ unimodular matrix $U$ over $Q$ such that $U M U^{t}$ is a diagonal matrix with $m$, say, nonzero entries $d_{1}, d_{2}, \ldots, d_{m}$. Thus rank $M=m$. $U$ can be extended to a $(|V(\Gamma)|-1) \times(|V(\Gamma)|-1)$ unimodular matrix $U_{1}$ so that

$$
U_{1} B_{\Gamma}(a) U_{1}^{t}=\left[\begin{array}{cccccc}
\tilde{a}_{22} & 0 & \cdots & 0 & \tilde{a}_{m+1} & \cdots \\
0 & d_{1} & & 0 & & \\
\vdots & & \ddots & & 0 & \\
0 & 0 & & d_{m} & & \\
\tilde{a}_{m+1} & & 0 & & 0 & \\
\vdots & & & & & \ddots
\end{array}\right]
$$

and

$$
U_{1} B_{\Gamma_{+}}(a) U_{1}^{t}=\left[\begin{array}{cccccc}
\tilde{a}_{22}+h & 0 & \cdots & 0 & \tilde{a}_{m+1} & \cdots \\
0 & d_{1} & & 0 & & \\
\vdots & & \ddots & & 0 & \\
0 & 0 & & d_{m} & & \\
\tilde{a}_{m+1} & & 0 & & 0 & \\
\vdots & & & & & \ddots
\end{array}\right] .
$$




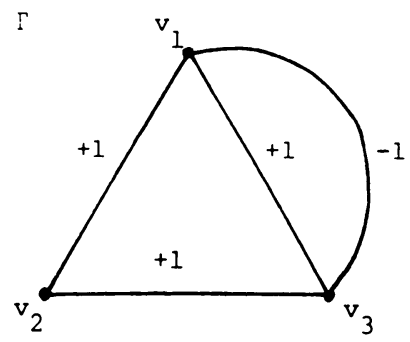

FIGURE 8.1

Case (I). $\tilde{a}_{m+i} \neq 0$ for some $i \geq 1$. Then $\sigma(\Gamma)=\sigma(M)=\sigma\left(\Gamma_{+}\right)$, and we are done.

Case (II). All $\tilde{a}_{m+i}=0$ (or rank $M=|V(\Gamma)|-2$ ). Then $\sigma(\Gamma)$ and $\sigma\left(\Gamma_{+}\right)$ are completely determined by the signs of $\tilde{a}_{22}$ and $\tilde{a}_{22}+h$. Since $h>0$, it follows that if $\tilde{a}_{22} \geq 0$, then $\tilde{a}_{22}+h>0$ and hence $\sigma(\Gamma) \leq \sigma\left(\Gamma_{+}\right) \leq \sigma(\Gamma)+1$. However, $\tilde{a}_{22}+h$ may be positive, even if $\tilde{a}_{22}<0$. Therefore, $\sigma\left(\Gamma_{+}\right)=\sigma(\Gamma)$, $\sigma(\Gamma)+1$, or $\sigma(\Gamma)+2$. This proves $(8.3)(1)$.

Now we return to a proof of Theorem 8.1. We consider $P$ and $N$ in $\Gamma$. Suppose that $P$ has $k$ connected components $P_{1}, P_{2}, \ldots, P_{k}$. Since $\Gamma$ is connected, there are $(k-1)$ negative isthmuses $e_{1}, \ldots, e_{k-1}$, each of which connects two components of $P$ and $\Gamma_{0}=P \cup\left\{e_{1}, \ldots, e_{k-1}\right\}$ is connected. Let $N_{i}$ be the graph consisting of $e_{i}$ and its ends. Then (7.4) shows that

$$
\sigma\left(\Gamma_{0}\right)=\sum_{i=1}^{k} \sigma\left(P_{i}\right)+\sum_{i=1}^{k-1} \sigma\left(N_{i}\right)
$$

Since $\sigma\left(P_{i}\right)=\left|V\left(P_{i}\right)\right|-1$ and $\sigma\left(N_{i}\right)=-1$, it follows that

$$
\begin{aligned}
\sigma\left(\Gamma_{0}\right) & =\sum_{i=1}^{k}\left\{\left|V\left(P_{i}\right)\right|-1\right\}-(k-1)=\sum_{i=1}^{k}\left|V\left(P_{i}\right)\right|-2 k+1 \\
& =|V(\Gamma)|-2 k+1
\end{aligned}
$$

Now in order to obtain $\Gamma$ from $\Gamma_{0}$, we have to add the remaining negative edges to $\Gamma_{0}$. However, by Lemma 8.2, $\sigma(\Gamma) \leq \sigma\left(\Gamma_{0}\right)=|V(\Gamma)|-2 k+1$. Since $r_{P}=k-1$, we have $\sigma(\Gamma) \leq \sigma\left(\Gamma_{0}\right)=|V(\Gamma)|-1-2 r_{P}$. This proves the right-side inequality in (8.2).

A proof of the other inequality is completely analogous but by taking $N$ instead of $P$, and hence the details will be omitted. This proves (8.1).

Remark 8.1. There is a "mixed" graph $\Gamma$ for which one of the equalities holds but not both. See Figure 8.1.

Example 8.1. The matrix of $\Gamma$ is

$$
B_{\Gamma}=\left[\begin{array}{ccc}
1 & -1 & 0 \\
-1 & 2 & -1 \\
0 & -1 & 1
\end{array}\right]
$$


Note that $\sigma(\Gamma)=2, v=3, r_{P}=s_{N}=0, s_{P}=r_{N}=1, p(\Gamma)=3$, and $n(\Gamma)=1$. Therefore, $r_{N}+s_{N}-n(\Gamma)=0 \neq \sigma(\Gamma)=2=p(\Gamma)-r_{P}-s_{P}$.

\section{DUAL GRAPHS}

So far, graphs have been completely arbitrary. However, in application to knot or link theory, we need only planar graphs. An advantage of dealing with planar graphs is the existence of the (geometric) dual graph. Since the dual graph also plays an important role in link theory, in this section we will prove some relationships between the invariants of a graph and its dual.

Let $\Gamma$ be a weighted planar graph, i.e., $\Gamma \subset S^{2}$. Let $\Gamma^{d}$ denote the dual graph of $\Gamma . V\left(\Gamma^{d}\right)$ and the set $F(\Gamma)$ of domains in $S^{2}-\Gamma$ are in one-toone correspondence, and $E\left(\Gamma^{d}\right)$ and $E(\Gamma)$ are in one-to-one correspondence in such a way that $e^{*} \in E\left(\Gamma^{d}\right)$ and its partner have exactly one point, not a vertex, in common. We define the weight of $e^{*}$ as that of its partner.
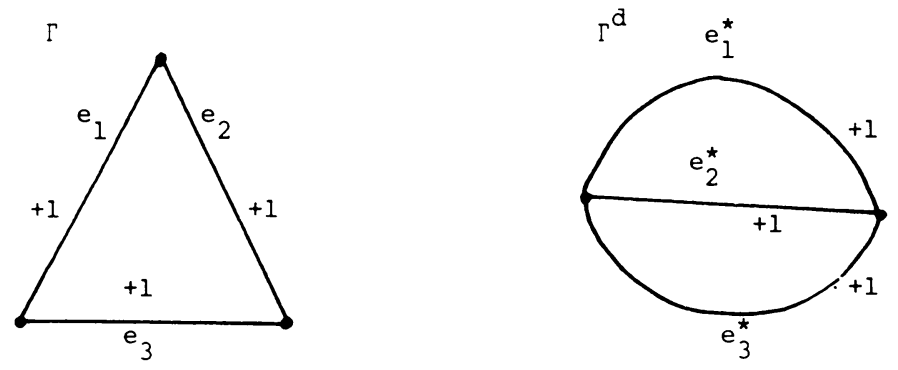

FIGURE 9.1

Example 9.1. The dual $G^{*}$ of a subgraph $G$ in $\Gamma$ is also defined. $G^{*}$ is a subgraph of $\Gamma^{d}$.

We begin with a simple proposition.

Proposition 9.1. Let $\Gamma$ be a connected planar graph. Then for any $r$ and $s$, $\mathscr{S}_{\Gamma}(r, s)$ and $\mathscr{S}_{\Gamma^{d}}(s, r)$ are in one-to-one correspondence.

Proof. Take $G \in \mathscr{S}_{\Gamma}(r, s)$, and consider the spanning complement $\hat{G}$ of $G$ in $\Gamma$. Let $\hat{G}^{*}$ be the dual of $\hat{G} . \hat{G}^{*}$ is a spanning subgraph of $\Gamma^{d}$. We claim that $\hat{G}^{*} \in \mathscr{S}_{\Gamma^{d}}(s, r)$, or $\beta_{0}\left(\hat{G}^{*}\right)=s+1$ and $\beta_{1}\left(\hat{G}^{*}\right)=r$. However, since $\beta_{i}\left(\hat{G}^{*}\right)=\beta_{i}\left(S^{2}-G\right), i=0,1$, our claim is exactly the consequence of the duality theorem:

$$
\beta_{0}\left(S^{2}-G\right)=\beta_{1}(G)+1 \text { and } \beta_{1}\left(S^{2}-G\right)=\beta_{0}(G)-1 \text {. Q.E.D. }
$$

Proposition 9.1 implies that the chromatic degrees of $\Gamma$ and $\Gamma^{d}$ are equal.

Proposition 9.2. $\mathrm{d}(\Gamma)=\mathrm{d}\left(\Gamma^{d}\right)$.

Proof. Let $G \in \mathscr{S}_{\Gamma}\left(r_{G}, s_{G}\right)$ be a maximal spanning subgraph of $\Gamma$, and hence $\mathbf{d}(\Gamma)=d(G)=r_{G}+s_{G}$. Since the dual $\hat{G}^{*}$ of the spanning complement of $G$ 
belongs to $\mathscr{S}_{\Gamma^{d}}\left(s_{G}, r_{G}\right)$, we see that $\mathbf{d}\left(\Gamma^{d}\right) \geq s_{G}+r_{G}=\mathbf{d}(\Gamma)$. Since $\left(\Gamma^{d}\right)^{d}=\Gamma$, the reverse inequality also holds, and hence $\mathbf{d}(\Gamma)=\mathbf{d}\left(\Gamma^{d}\right)$. Q.E.D.

Proposition 9.1 also implies a relationship between $P_{\Gamma}(x, y, z)$ and $P_{\Gamma^{d}}(x, y, z)$.

Theorem 9.3. $x^{p(\Gamma)-n(\Gamma)} P_{\Gamma}\left(x^{-1}, y, z\right)=P_{\Gamma^{d}}(x, z, y)$.

Proof. By Proposition 9.1, $\mathscr{S}_{\Gamma}(r, s)$ and $\mathscr{S}_{\Gamma^{d}}(s, r)$ are in 1-to-1 correspondence by $G \leftrightarrow \hat{G}^{*}$. The term associated with $G$ in $P_{\Gamma}(x, y, z)$ is $x^{p(G)-n(G)} y^{r} z^{s}$ while the term associated with $\hat{G}^{*}$ in $P_{\Gamma^{d}}(x, y, z)$ is $x^{p\left(\hat{G}^{*}\right)-n\left(\hat{G}^{*}\right)} y^{s} z^{r}$. Since $p\left(\hat{G}^{*}\right)+p(G)=p(\Gamma)$ and $n\left(\hat{G}^{*}\right)+n(G)=n(\Gamma)$, we have

$$
x^{p(\Gamma)-n(\Gamma)} x^{-(p(G)-n(G))}=x^{p\left(\hat{G}^{*}\right)-n\left(\hat{G}^{*}\right)} .
$$

This proves Theorem 9.3.

Example 9.2. Let $\Gamma$ and $\Gamma^{d}$ be the graphs in Figure 9.1. Then $P_{\Gamma}(x, y, z)=$ $3 x^{2}+3 x y+x^{3} z+y^{2}, P_{\Gamma^{d}}(x, y, z)=3 x+y+3 x^{2} z+x^{3} z^{2}$, and $x^{3} P_{\Gamma}\left(x^{-1}, y, z\right)$ $=P_{\Gamma^{d}}(x, z, y)$.

There is no easy way to obtain the matrix of the dual $\Gamma^{d}$ from the matrix of $\Gamma$. Nevertheless, many invariants of $\Gamma$ and $\Gamma^{d}$ are related in very simple manners. In fact, we can prove the following three theorems.

Theorem 9.4. $\operatorname{det}(\Gamma)=(-1)^{n(\Gamma)} \operatorname{det}\left(\Gamma^{d}\right)$.

Proof. As we have seen in Proposition 9.1, $\mathscr{S}_{\Gamma}(0,0)$ and $\mathscr{S}_{\Gamma^{d}}(0,0)$ are in one-to-one correspondence by $G \leftrightarrow \hat{G}^{*}$. Since, by (7.2),

$$
\operatorname{det}(\Gamma)=\sum_{G \in \mathscr{S}_{\Gamma}(0,0)}(-1)^{n(G)} \text { and } \operatorname{det}\left(\Gamma^{d}\right)=\sum_{\hat{G}^{*} \in \mathscr{S}_{\Gamma^{d}}(0,0)}(-1)^{n\left(\hat{G}^{*}\right)},
$$

and since $n\left(\hat{G}^{*}\right)=n(\Gamma)-n(G)$, it follows that

$$
\begin{aligned}
(-1)^{n(\Gamma)} \operatorname{det}\left(\Gamma^{d}\right) & =\sum_{G^{*} \in \mathscr{S}_{\Gamma}(0,0)}(-1)^{n\left(\hat{G}^{*}\right)+n(\Gamma)} \\
& =\sum_{G \in \mathscr{S}_{\Gamma}(0,0)}(-1)^{n(G)}=\operatorname{det}(\Gamma) \text {. Q.E.D. }
\end{aligned}
$$

The nullity of an $m \times m$ matrix $B$, denoted null $(B)$, is defined as $m-$ $\operatorname{rank}(B)$.

Theorem 9.5. Let $B_{\Gamma}$ and $B_{\Gamma^{d}}$ be the matrices of $\Gamma$ and $\Gamma^{d}$, respectively. Then $\operatorname{null}\left(B_{\Gamma}\right)=\operatorname{null}\left(B_{\Gamma^{d}}\right)$.

Theorem 9.6. $\sigma(\Gamma)+\sigma\left(\Gamma^{d}\right)=p(\Gamma)-n(\Gamma)$.

Proofs of Theorems 9.5 and 9.6 will be given in Appendix (I), because our proofs depend on a topological interpretation of $B_{\Gamma}$ discussed in $\S \S 12-15$. It 
would, however, be nice to find direct proofs of these theorems without referring to topological results. At present, we are able to prove Theorem 9.6 directly only when $\operatorname{det}(\Gamma) \neq 0$.

\section{Periodic GRAPHS}

If a graph has a certain special property, the polynomial may be of a special form. In this section, we will discuss the polynomial of a graph on which a cyclic group acts in a certain way.

Let $\left(\Gamma, f_{\Gamma}\right)$ be a weighted graph. An automorphism $\phi$ of $\Gamma$ consists of two permutations

such that

$$
\phi_{1}: V(\Gamma) \rightarrow V(\Gamma) \text { and } \phi_{2}: E(\Gamma) \rightarrow E(\Gamma)
$$

$(10.1)(1)$ if $a$ and $b$ are ends of an edge $e, \phi_{1}(a)$ and $\phi_{1}(b)$ are ends of $\phi_{2}(e)$;

(2) for any edge $e, f_{\Gamma}(e)=f_{\Gamma}\left(\phi_{2}(e)\right)$.

An automorphism $\phi$ of $\Gamma$ induces and is induced by a simplicial automorphism $\phi^{*}$ of a geometric realization of $\Gamma$ as a 1-dimensional $C W$-complex. Therefore, we can define two different types of fixed-point sets.

$F\left(\phi^{*}\right)$ denotes the set of fixed points under $\phi^{*}$, while $F(\phi)$ denotes the set of those vertices and edges of $\Gamma$ which are kept fixed under $\phi$, setwise.

An automorphism $\phi$ of $\Gamma$ is called periodic (of period $k$ ) if $\phi^{k}=$ id and $F\left(\phi^{*}\right)=F(\phi)$ and, moreover, $F(\phi)$ is either empty or a subset of $V(\Gamma)$. If $\Gamma$ admits a periodic automorphism, we say that $\Gamma$ has period $k$.

Suppose that $\Gamma$ has period $k$ and let $\phi$ be a periodic automorphism. Then by identifying the vertices $v, \phi_{1}(v), \ldots, \phi_{1}^{k-1}(v)$ to a single vertex $\tilde{v}$, and edges $e, \phi_{2}(e), \ldots, \phi_{2}^{k-1}(e)$ to a single edge $\tilde{e}$, we obtain a new (weighted) graph $\tilde{\Gamma}$ called the quotient graph, denoted by $\Gamma / \phi$.

From this construction, we see immediately

$$
\begin{aligned}
& \text { (1) } k\{|V(\Gamma / \phi)|-|F(\phi)|\}=|V(\Gamma)|-|F(\phi)| ; \\
& \text { (2) } k|E(\Gamma / \phi)|=|E(\Gamma)| .
\end{aligned}
$$

In this section, we will prove

Theorem 10.1. Suppose that $\Gamma$ is a connected graph having a prime period $q$, $q \geq 2$. Let $\phi$ be a periodic automorphism of $\Gamma$ with period $q$. Then

$$
P_{\Gamma}(x, y, z) \equiv\left[P_{\Gamma / \phi}(x, y, z)\right]^{q} \quad \bmod \left(q, y^{q-1}-1, z^{q-1}-1\right)
$$

and hence

$$
P_{\Gamma}(x, y, z) \equiv P_{\Gamma / \phi}\left(x^{q}, y, z\right) \quad \bmod \left(q, y^{q-1}-1, z^{q-1}-1\right) .
$$

Remark 10.1. (1) Theorem 10.1 need not be true if period $q$ is not a prime.

(2) If $F(\phi)$ contains edges of $\Gamma$, then Theorem 10.1 is not true, as is shown in the example below. 

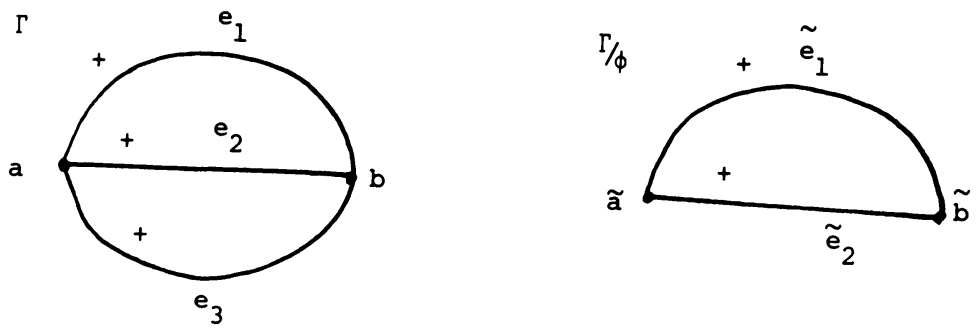

Figure 10.1

Example 10.1. Let $\Gamma$ be a graph in Figure 10.1. Let $\phi_{1}=\mathrm{id}$ and $\phi_{2}: e_{1} \rightarrow e_{3}$, $e_{2} \rightarrow e_{2}, e_{3} \rightarrow e_{1}$. Then $\phi=\left(\phi_{1}, \phi_{2}\right)$ is an automorphism of period 2 , and $F(\phi)=\left\{a, b, e_{2}\right\}$. Now $P_{\Gamma}(x, y, z)=3 x+3 x^{2} z+y+x^{3} z^{2}$, while $P_{\Gamma / \phi}(x, y, z)=2 x+y+x^{2} z$, and hence $P_{\Gamma}(x, y, z) \not \equiv P_{\Gamma / \phi}\left(x^{2}, y, z\right)$ $\bmod (2, y-1, z-1)$.

Proof of Theorem 10.1. Write

$$
P_{\Gamma}(x, y, z)=\sum_{0 \leq r, s<\infty} \sum_{\mathscr{S}_{\Gamma}(r, s) \ni G} x^{p(G)-n(G)} y^{r} z^{s}
$$

and for $\tilde{\Gamma}=\Gamma / \phi$

$$
P_{\Gamma}(x, y, z)=\sum_{0 \leq \tilde{r}, \tilde{s}<\infty} \sum_{\mathscr{S}_{\tilde{r}}(\tilde{r}, \tilde{s}) \ni \tilde{G}} x^{p(\tilde{G})-n(\tilde{G})} y^{\tilde{r}} z^{\tilde{s}}
$$

Take $G \in \mathscr{S}_{\Gamma}\left(r_{G}, s_{G}\right)$.

Case (I). $\phi(G) \neq G$, setwise. Since $G$ is isomorphic to $\phi(G)$,

$$
\phi^{i}(G) \in \mathscr{S}_{\Gamma}\left(r_{G}, s_{G}\right) \text { for } 0 \leq i \leq q-1 .
$$

Therefore $\mathscr{S}_{\Gamma}\left(r_{G}, s_{G}\right)$ contains $q$ isomorphic (but distinct) spanning subgraphs, $G, \phi(G), \ldots, \phi^{q-1}(G)$. Since the terms in $P_{\Gamma}(x, y, z)$ associated with $\phi^{i}(G)$ are identical for all $i$, the sum of these $q$ terms vanishes modulo $q$. Therefore we need only consider the following case.

Case (II). $\phi(G)=G$, setwise. Let $G_{1}, G_{2}, \ldots, G_{l}$ be connected components of $G$. The collection $\left\{G_{1}, G_{2}, \ldots, G_{l}\right\}$ is grouped into disjoint sets $A_{1}=$ $\left\{G_{1}, G_{2}, \ldots, G_{q}\right\}, A_{2}=\left\{G_{q+1}, \ldots, G_{2 q}\right\}, \ldots, A_{m}=\left\{G_{(m-1) q+1}, \ldots, G_{m q}\right\}$, and $B=\left\{G_{m q+1}, \ldots, G_{l}\right\}$ in such a way that

(1) $\phi\left(G_{t q+i}\right)=G_{t q+i+1}$, for $0 \leq t<m$ and $1 \leq i \leq q-1$;

(2) $\phi\left(G_{m q+i}\right)=G_{m q+i}$, setwise.

Note that $G_{m q+i}$ may be a subset of $F(\phi)$.

Now the term in $P_{\Gamma}(x, y, z)$ associated with $G$ is

$$
x^{\sum_{i}\left\{p\left(G_{i}\right)-n\left(G_{i}\right)\right\}} y^{l-1} z^{\sum_{i} s\left(G_{i}\right)} .
$$


Since

$$
\begin{array}{rlrl}
p\left(G_{t q+i}\right) & =p\left(G_{t q+i+1}\right), & n\left(G_{t q+i}\right)=n\left(G_{t q+i+1}\right), \quad 0 \leq t<m, \text { and } \\
p\left(G_{m q+i}\right) & \equiv n\left(G_{m q+i}\right) \equiv 0 & (\bmod q), &
\end{array}
$$

we see that

$$
\begin{aligned}
& \sum_{i=1}^{l}\left[p\left(G_{i}\right)-n\left(G_{i}\right)\right] \\
& \quad=q \sum_{t=0}^{m-1}\left\{p\left(G_{t q+1}\right)-n\left(G_{t q+1}\right)\right\}+q \sum_{i>0}\left\{p\left(\tilde{G}_{m q+i}\right)-n\left(G_{m q+i}\right)\right\} \\
& \quad=q\{p(\tilde{G})-n(\tilde{G})\},
\end{aligned}
$$

where $\tilde{G}=G / \phi$ and $\tilde{G}_{i}=G_{i} / \phi$. Furthermore, $\tilde{G}$ has exactly $m+(l-m q)$ components and hence $r_{\tilde{G}}=l+m-m q-1$ and $r_{G}=l-1$. Therefore, $r_{\tilde{G}} \equiv r_{G}$ $(\bmod q-1)$.

Now it remains to show that

$$
s_{G} \equiv S_{\tilde{G}} \quad(\bmod q-1) .
$$

Since $\phi\left(G_{t q+i}\right)=G_{t q+i+1}$ for $0 \leq t<m$ and $1 \leq i \leq q-1$, it follows that $s_{G_{t q+1}}=s_{G_{t q+t+1}}$ and hence

$$
s_{G}=\sum_{i=1}^{l} s_{G_{l}}=q\left(\sum_{t=0}^{m-1} s_{G_{t q+1}}\right)+s_{G_{m q+1}}+\cdots+s_{G_{l}},
$$

while

$$
s_{\tilde{G}}=s_{G_{1}}+s_{G_{q+1}}+\cdots+s_{G_{(m-1) q+1}}+s_{\tilde{G}_{m q+1}}+\cdots+s_{\tilde{G}_{l}} \cdot
$$

Therefore, it suffices to prove that for $i=m q+1, \ldots, l$

$$
s_{\tilde{G}_{i}} \equiv s_{G_{i}} \bmod (q-1) \text {. }
$$

Suppose that $G_{i}$ contains $h$ vertices fixed under $\phi$. Then (10.2) shows that $\left|V\left(G_{i}\right)\right|-h=q\left\{\left|V\left(\tilde{G}_{i}\right)\right|-h\right\}$ and $\left|E\left(G_{i}\right)\right|=q\left|E\left(\tilde{G}_{i}\right)\right|$, and hence $\left|V\left(G_{i}\right)\right| \equiv$ $\left|V\left(\tilde{G}_{i}\right)\right| \bmod (q-1)$ and $\left|E\left(G_{i}\right)\right| \equiv\left|E\left(\tilde{G}_{i}\right)\right| \bmod (q-1)$. Since $G_{i}$ and $\tilde{G}_{i}$ are connected, $\beta_{0}\left(G_{i}\right)=\beta_{0}\left(\tilde{G}_{i}\right)=1$, and hence

$$
\begin{aligned}
s_{G_{t}} & =\beta_{1}\left(G_{i}\right)=\beta_{0}\left(G_{i}\right)-\left|V\left(G_{i}\right)\right|+\left|E\left(G_{i}\right)\right| \\
& \equiv 1-\left|V\left(\tilde{G}_{i}\right)\right|+\left|E\left(\tilde{G}_{i}\right)\right| \bmod (q-1) \\
& =s_{\tilde{G}_{t}} .
\end{aligned}
$$

This proves (10.8) and hence Theorem 10.1 .

The following corollaries are immediate consequences of Theorem 10.1.

Corollary 10.2. Under the assumption of Theorem 10.1,

$$
P_{\Gamma}(x, y, z) \equiv g\left(x^{q}, y, z\right) \quad(\bmod q),
$$

where $g(x, y, z)$ is an integer polynomial in $x^{ \pm 1}, y$, and $z$. 
Corollary 10.3. Let $\Gamma$ be a connected simple graph and let $\operatorname{Aut}(\Gamma)$ denote the group of automorphisms of $\Gamma$. Suppose that Aut $(\Gamma)$ has an element of order $q$, a prime, which does not keep any edge fixed. Then

$$
P_{\Gamma}(x, y, z) \equiv g\left(x^{q}, y, z\right) \quad(\bmod q)
$$

for some integer polynomial $g(x, y, z)$.

Although a relationship between $\sigma(\Gamma)$ and $\sigma(\Gamma / \phi)$ is expected, it would be generally very complicated. We only state such a relationship for connected positive or negative graphs.

Proposition 10.4. Let $\Gamma$ be a connected positive graph. Suppose that $\Gamma$ is periodic with an arbitrary period $m(\geq 2)$. Then

(1) $\sigma(\Gamma)-m \sigma(\Gamma / \phi)=(m-1)[1-|F(\phi)|]$,

and hence

$$
\text { (2) } \sigma(\Gamma) \equiv \sigma(\Gamma / \phi) \quad \bmod (m-1) \text {. }
$$

A proof follows immediately from Proposition 7.3 and (10.2).

Remark 10.2. (1) An application of Theorem 10.1 and Corollary 10.2 to link theory can be found in [M5, Tr1]. (2) Some results in link theory related to Proposition 10.4 can be found in [M2] or [GLM].

\section{CONGRUENT GRAPHS}

Let $n$ be a nonnegative integer. Two weighted graphs $\Gamma_{1}$ and $\Gamma_{2}$ are said to be congruent $(\bmod n)$, denoted $\Gamma_{1} \equiv \Gamma_{2}(\bmod n)$, if $\left|V\left(\Gamma_{1}\right)\right|=\left|V\left(\Gamma_{2}\right)\right|$ and the matrix $B_{\Gamma_{1}}$ is congruent $(\bmod n)$ to $B_{\Gamma_{2}}$, after changing rows and columns simultaneously, if necessary.

\section{Example 11.1.}
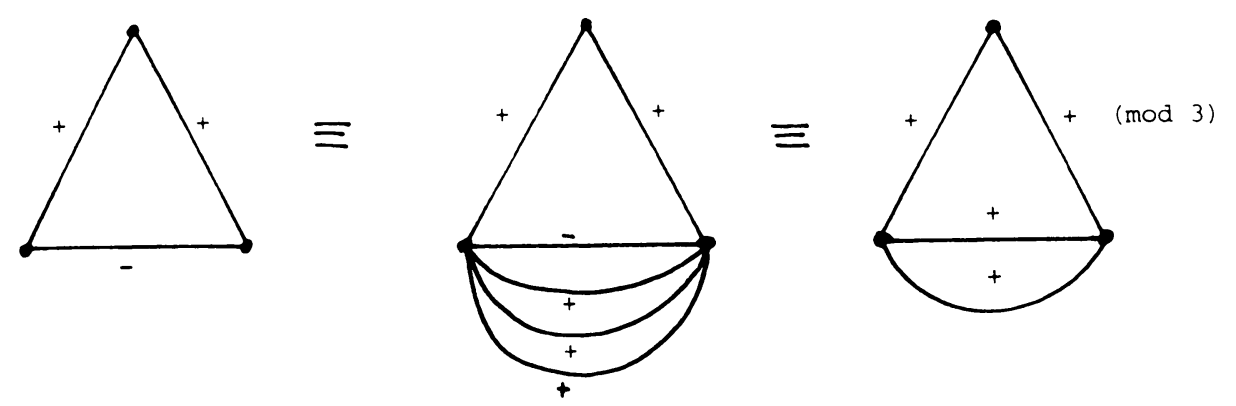

FIGURE 11.1 
Proposition 11.1. For any weighted graph $\Gamma$,

(1) $\Gamma \equiv V_{\Gamma}(\bmod 1)$.

(2) For any $n \geq 1, \Gamma$ is congruent $(\bmod n)$ to a positive (or negative) graph.

(3) $\Gamma$ is congruent (mod 3) to a simple graph.

(4) $\Gamma_{1} \equiv \Gamma_{2}(\bmod 0)$, if $\Gamma_{1}$ and $\Gamma_{2}$ are isomorphic. The converse need not be true.

A proof is obvious.

Let $g(x, y, z)$ and $h(x, y, z)$ be two polynomials in $\mathbf{Z}\left[x^{ \pm 1}, y, z\right]$ and $I$ an ideal in $\mathbf{Z}\left[x^{ \pm 1}, y, z\right]$. We denote $g(x, y, z) \doteq h(x, y, z)(\bmod I)$ if $g(x, y, z) \equiv$ $\pm x^{k} h(x, y, z)(\bmod I)$ for some integer $k$.

Now the main theorem of this section is the following theorem.

Theorem 11.2. Suppose $\Gamma_{1} \equiv \Gamma_{2}(\bmod n)$. Then

$$
P_{\Gamma_{1}}(x, y, z) \doteq P_{\Gamma_{2}}(x, y, z) \quad(\bmod I),
$$

where $I$ is the ideal in $\mathbf{Z}\left[x^{ \pm 1}, y, z\right]$ generated by $\left(x^{2 n}-(-1)^{n}\right) /\left(x^{2}+1\right)$ and $x+x^{-1}+z$.

Proof. We know (Remark 7.1) that the addition and removal of a loop does not change the matrix but does change the polynomial. Let $\Gamma^{\prime}$ be the graph obtained from $\Gamma$ by deleting a loop $e$. Let $\varepsilon=f_{\Gamma}(e)$. Then we have from (2.2)(II)(ii) that $P_{\Gamma}(x, y, z)=\left(1+x^{\varepsilon} z\right) P_{\Gamma^{\prime}}(x, y, z)$. Since $1+x^{\varepsilon} z \equiv 1-x^{\varepsilon}\left(x+x^{-1}\right)=-x^{2 \varepsilon}$ $(\bmod I)$, it follows that $P_{\Gamma}(x, y, z) \doteq P_{\Gamma^{\prime}}(x, y, z)(\bmod I)$. Therefore we can eliminate all loops from $\Gamma$ within the congruence class of the polynomial.

Next, suppose that $\Gamma$ contains multiple edges $e_{1}$ and $e_{2}$ with the different weights. Let $\Gamma^{\prime}=\Gamma-\left\{e_{1}, e_{2}\right\}$. Then

$$
P_{\Gamma}(x, y, z) \equiv P_{\Gamma^{\prime}}(x, y, z) \quad(\bmod I) .
$$

To prove (11.2), let $f_{\Gamma}\left(e_{1}\right)=+1$ and $f_{\Gamma}\left(e_{2}\right)=-1$. Then (2.2)(II)(i) shows that

$$
\begin{aligned}
P_{\Gamma}(x, y, z) & =P_{\Gamma-\left(e_{1}\right)}(x, y, z)+x P_{\Gamma /\left(e_{1}\right)}(x, y, z) \\
& =P_{\Gamma-\left\{e_{1}, e_{2}\right\}}(x, y, z)+x^{-1} P_{\left(\Gamma-\left(e_{1}\right)\right) /\left(e_{2}\right)}+x P_{\Gamma /\left(e_{1}\right)}(x, y, z) .
\end{aligned}
$$

Note that $\Gamma^{\prime}=\Gamma-\left\{e_{1}, e_{2}\right\}$ and $\Gamma /\left(e_{1}\right)$ is exactly the graph $\left(\Gamma-\left(e_{1}\right)\right) /\left(e_{2}\right)$ to which one negative loop is attached. Therefore,

$$
P_{\Gamma /\left(e_{1}\right)}(x, y, z)=\left(1+x^{-1} z\right) P_{\left(\Gamma-\left(e_{1}\right)\right) /\left(e_{2}\right)} .
$$

Since $x\left(1+x^{-1} z\right)=x+z \equiv-x^{-1}(\bmod I)$, the last two terms in $(11.3)$ are canceled $(\bmod I)$, which proves $(11.2)$.

Finally, let $\Gamma^{\prime}$ be the graph obtained from $\Gamma$ by deleting $n$ multiple edges $e_{1}, e_{2}, \ldots, e_{n}$, each of which has the same weight $\varepsilon$. Then, to complete the proof of Theorem 11.2, it suffices to show that

$$
P_{\Gamma}(x, y, z) \equiv P_{\Gamma^{\prime}}(x, y, z) \quad(\bmod I) .
$$


Let $\Gamma_{k}=\Gamma-\left\{e_{k+1}, \ldots, e_{n}\right\}, k=0,1, \ldots, n$, and hence $\Gamma_{0}=\Gamma^{\prime}$ and $\Gamma_{n}=\Gamma$. Furthermore, let $\Gamma_{-1}=\left(\Gamma-\left\{e_{1}, \ldots, e_{n-1}\right\}\right) /\left(e_{n}\right)$.

Now by an easy inductive argument, we can show that

$$
P_{\Gamma_{k+1}}(x, y, z)=P_{\Gamma_{k}}(x, y, z)+x^{\varepsilon}\left(1+x^{\varepsilon} z\right)^{k} P_{\Gamma_{-1}}(x, y, z) \text { for } 0 \leq k \leq n-1,
$$

and hence

$P_{\Gamma}(x, y, z)=P_{\Gamma^{\prime}}(x, y, z)+P_{\Gamma_{-1}}(x, y, z)\left\{1+\left(1+x^{\varepsilon} z\right)+\cdots+\left(1+x^{\varepsilon} z\right)^{n-1}\right\} x^{\varepsilon}$.

However, we see that

$$
1+\left(1+x^{\varepsilon} z\right)+\cdots+\left(1+x^{\varepsilon} z\right)^{n-1}=\frac{\left(1+x^{\varepsilon} x\right)^{n}-1}{1+x^{\varepsilon} z_{1}} \equiv \frac{\left(-x^{2 \varepsilon}\right)^{n}-1}{-x^{2 \varepsilon}-1} \equiv 0 \quad(\bmod I),
$$

and hence $P_{\Gamma}(x, y, z) \equiv P_{\Gamma},(x, y, z)(\bmod I)$. This proves $(11.4)$ and hence Theorem 11.2.

The following corollaries are immediate consequences of Theorem 11.2.

Corollary 11.3. If $\Gamma_{1} \equiv \Gamma_{2}(\bmod 0)$, then

$$
P_{\Gamma_{1}}(x, y, z) \doteq P_{\Gamma_{2}}(x, y, z) \quad \bmod \left(x+x^{-1}+z\right) \text {. }
$$

Corollary 11.4. Suppose $\Gamma_{1} \equiv \Gamma_{2}(\bmod n)$. Let $\xi$ be a primitive $k$ th root of unity, where $k=n$ or $2 n$ according as $n$ is even or odd. Then for any complex number $\zeta$,

$$
\left|P_{\Gamma_{1}}\left(\xi, \zeta,-\left(\xi+\xi^{-1}\right)\right)\right|=\left|P_{\Gamma_{2}}\left(\xi, \zeta,-\left(\xi+\xi^{-1}\right)\right)\right| .
$$

Some results in link theory relevant to Corollary 11.4 can be found in [P1].

\section{APPLICATION TO LINK THEORY (I)-JONES POLYNOMIAL}

The last four sections of this paper will be devoted to the application of results in $\S \S 2-9$ to knots or links in $S^{3}$.

Given a knot or link $L \subset S^{3}$, we project it into $S^{2}$. The image of $L$ is called a link diagram of $L$. To be more precise, a link diagram $\tilde{L}$ of $L$ is an image of $L$ in which we specify which arc is running over the other. We do not distinguish between a diagram and the image of $L$, but we hope that it does not cause any confusion to the readers.

We may assume without loss of generality that $\tilde{L}$ intersects itself transversely and has only finitely many crossings.

$\tilde{L}$ divides $S^{2}$ into finitely many domains, which will be classified as shaded or unshaded. No two shaded or unshaded domains have an edge in common.

There are exactly two such classifications (or shadings) of $S^{2}-\tilde{L}$. For convenience, one is called the opposite of the other.

We now construct a planar graph $\Gamma$ from $\tilde{L}$ and shading $\rho$.

Take a point $v_{i}$ from each unshaded domain $D_{i}$. These points form the set of vertices $V(\Gamma)$ of $\Gamma$. If the boundaries of $D_{i}$ and $D_{j}$ intersect at $k$, say, crossings $c_{l_{1}}, c_{l_{2}}, \ldots, c_{l_{k}}$, then we form multiple edges $e_{l_{1}}, e_{l_{2}}, \ldots, e_{l_{k}}$ on $S^{2}$ 

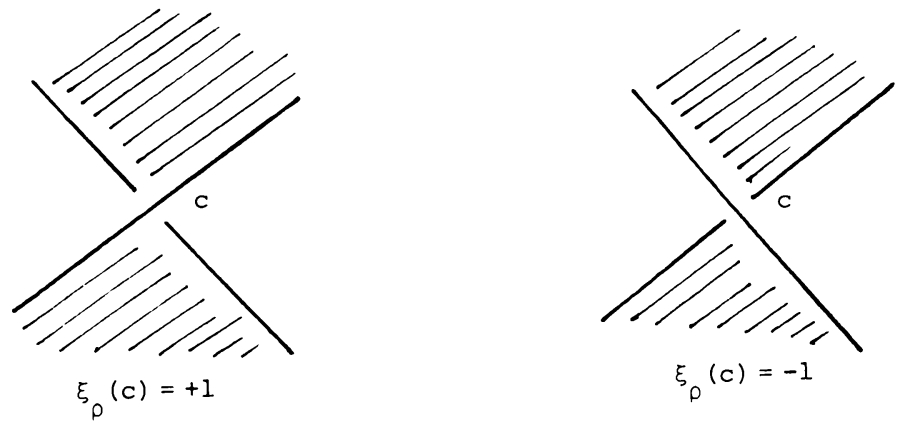

FIGURE 12.1

with common ends $v_{i}$ and $v_{j}$, where each edge $e_{l_{m}}$ passes through a crossing $c_{l_{m}}, m=1,2, \ldots, k$.

To define a weight of an edge, first we define the index $\xi_{\rho}(c)$ to each crossing $c$ of a link diagram as shown in Figure 12.1. We should note that $\xi_{\rho}(c)$ depends on the shading of the domains. In fact, if $\rho^{\prime}$ is opposite in shading to $\rho$, then $\xi_{\rho^{\prime}}(c)=-\xi_{\rho}(c)$.

Since each edge $e$ of $\Gamma$ passes through exactly one crossing, $c$ say, of $\tilde{L}$, the weight $f_{\Gamma}(e)$ will be well defined as $f_{\Gamma}(e)=\xi_{\rho}(c)$. The resulting weighted planar graph is called the graph of a link with respect to $\tilde{L}$ and shading $\rho$ and is denoted by $\Gamma(\tilde{L}, \rho)$ (or $\Gamma(\tilde{L}), \Gamma_{\tilde{L}}$, or simply $\left.\Gamma\right) . \Gamma(\tilde{L}, \rho)$ depends not only on $\tilde{L}$ but also on shading.

If we use the opposite shading $\rho^{\prime}$, the resulting graph $\Gamma\left(\tilde{L}, \rho^{\prime}\right)$ is the opposite graph of the dual of $\Gamma(\tilde{L}, \rho)$.

Conversely, given a weighted planar graph $\Gamma$, one can construct uniquely the link diagram $\tilde{L}$ of a link and can choose shading $\rho$ so that $\Gamma(\tilde{L}, \rho)$ is exactly $\Gamma$.

Now each vertex and each edge of $\Gamma(\tilde{L}, \rho)$ correspond, respectively, to an unshaded domain and a crossing of $\tilde{L}$. If $\Gamma(\tilde{L}, \rho)$ is a reduced graph, a diagram $\tilde{L}$ is said to be reduced.

Example 12.1. If $\Gamma(\tilde{L}, \rho)$ is a positive or negative graph, $L$ is called an alternating diagram, and a link that has an alternating diagram is called an alternating link. A change of shading results in the opposite graph of the dual of $\Gamma$. Therefore, if $\Gamma(\tilde{L}, \rho)$ is a negative graph, then $\Gamma\left(\tilde{L}, \rho^{\prime}\right)$ is a positive graph, where $\rho^{\prime}$ is the opposite of $\rho$, and thus if $\tilde{L}$ is an alternating diagram, we may assume without loss of generality that $\Gamma(\tilde{L}, \rho)$ is a positive graph.

Now, given a link $L$, we can define the polynomial $P_{\Gamma(\tilde{L}, \rho)}(x, y, z)$, or simply $P_{\tilde{L}}(x, y, z)$, of the graph $\Gamma(\tilde{L}, \rho)$. Since a link $L$ can have many different link diagrams, we may have many different polynomials. In other words, the 

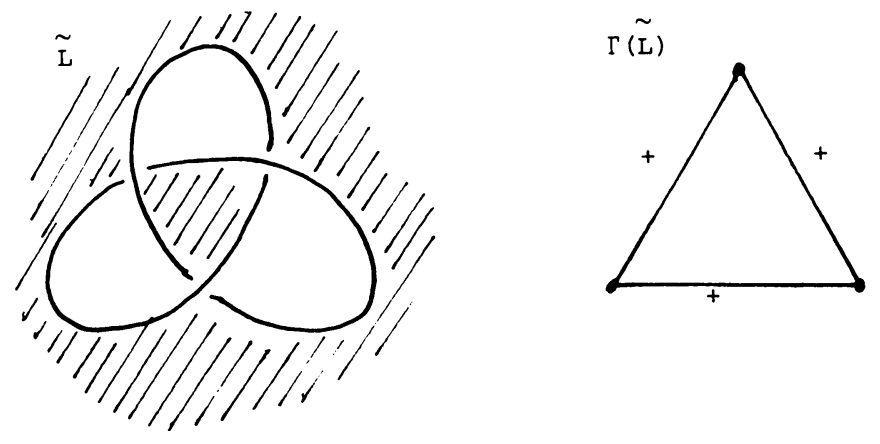

Figure 12.2
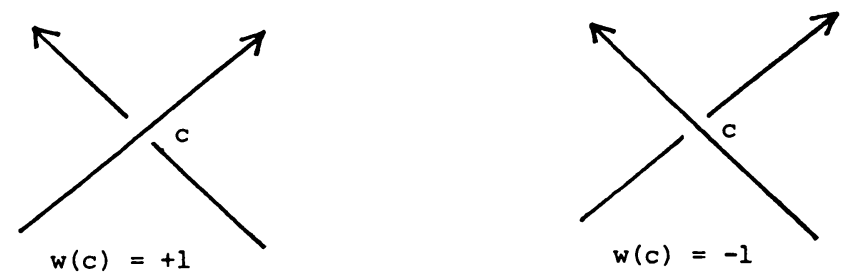

FIGURE 12.3

polynomial obtained from $\Gamma(\tilde{L}, \rho)$ is not necessarily unique to each $L$. However, we can define a unique polynomial to each $L$ no matter which diagram we use. This polynomial depends on the orientation of a link $L$.

Suppose that we are given an oriented link $L$. The orientation of $L$ induces the orientation of a diagram $\tilde{L}$. We then define the second index $w(c)$, called the $t w i s t$ or writhe, at each crossing $c$ as is shown in Figure 12.3. The sum of all writhes on $\tilde{L}, w(c)=\sum_{c \in \tilde{L}} w(c)$, is called the writhe of $\tilde{L}$. We should note that the writhe is independent of shading.

Let $P_{\Gamma(\tilde{L}, \rho)}(x, y, z)$ be the polynomial of the graph $\Gamma(\tilde{L}, \rho)$ associated with an (oriented) diagram $\tilde{L}$.

Theorem 12.1 (Kauffman [K1]). Let $p=p(\Gamma(\tilde{L}, \rho))$ and $n=n(\Gamma(\tilde{L}, \rho))$. Then

$$
\mathbf{V}_{L}(x)=(-1)^{p-n} x^{-(p-n-3 w(\tilde{L})) / 2} P_{\tilde{L}}\left(x,-\left(x+x^{-1}\right)-\left(x+x^{-1}\right)\right)
$$

is independent of a diagram $\tilde{L}$ and $\rho$ and depends only on the ambient isotopy type of $L$. In other words, $\mathbf{V}_{L}(x)$ is an invariant of $L$.

For a proof, we refer to [K1]. However, we should note that $\mathbf{V}_{L}(x)$ does not depend on shading. In fact, if we change a shading, the resulting graph is the opposite graph $\hat{\Gamma}^{d}$ of the dual $\Gamma^{d}$ of $\Gamma$. Therefore, $p\left(\hat{\Gamma}^{d}\right)=n(\Gamma)$ and $n\left(\hat{\Gamma}^{d}\right)=p(\Gamma)$. Furthermore, Corollary 2.3 and Theorem 9.3 imply that 


$$
\begin{gathered}
P_{\hat{\Gamma}^{d}}(x, y, z)=P_{\Gamma^{d}}\left(x^{-1}, y, z\right)=x^{-[p(\Gamma)-n(\Gamma)]} P_{\Gamma}(x, y, z), \text { and hence } \\
(-1)^{p\left(\hat{\Gamma}^{d}\right)-n\left(\hat{\Gamma}^{d}\right)} x^{-\left(p\left(\hat{\Gamma}^{d}\right)-n\left(\hat{\Gamma}^{d}\right)-3 w(\tilde{L})\right) / 2} P_{\hat{\Gamma}^{d}}(x, y, z) \\
=(-1)^{p(\Gamma)-n(\Gamma)} x^{-(n(\Gamma)-p(\Gamma)-3 w(\tilde{L})) / 2-[p(\Gamma)-n(\Gamma)]} P_{\Gamma}(x, y, z) \\
=(-1)^{p(\Gamma)-n(\Gamma)} x^{-(p(\Gamma)-n(\Gamma)-3 w(\tilde{L})) / 2} P_{\Gamma}(x, y, z),
\end{gathered}
$$

which shows that $\mathbf{V}_{L}(x)$ does not depend on shading.

Now since $p-n-3 w(\tilde{L}) \equiv p+n-w(\tilde{L}) \equiv 0(\bmod 2), \mathbf{V}_{L}(x)$ is a polynomial on $x^{ \pm 1}$, and the polynomial $V_{L}(x)$ :

$$
\begin{aligned}
V_{L}(x) & =\mathbf{V}_{L}\left(x^{1 / 2}\right) \\
& =(-1)^{p-n} x^{-(p-n-3 w(\tilde{L})) / 4} P_{\tilde{L}}\left(x^{1 / 2},-\left(x^{1 / 2}+x^{-1 / 2}\right),-\left(x^{1 / 2}+x^{-1 / 2}\right)\right)
\end{aligned}
$$

is called the Jones polynomial of a link $L$. This (due to Kauffman) is an important alternate definition of the Jones polynomial [J1].

For example, the following theorem due to M. B. Thistlethwaite is an immediate consequence of this alternate definition of $V_{L}(x)$.

Theorem 12.2 [Th1]. The Jones polynomial of a nonsplit alternating link $L$ is alternating. In other words, if $V_{L}(x)=\sum_{-\infty<k<\infty} c_{k} x^{k}$, then $c_{k} c_{k+1} \leq 0$ for any $k,-\infty<k<\infty$.

Proof. Let $\Gamma$ be the positive graph associated with a reduced alternating diagram $\tilde{L}$ of $L$. Since $\Gamma$ is positive, Proposition 2.6 shows that

$$
P_{\Gamma}(x, y, z)=x^{v-1} \sum_{0 \leq r, s<\infty} a_{r, s}\left(\frac{y}{x}\right)^{r}(x z)^{s},
$$

where $v=|V(\Gamma)|$ and $a_{r, s}=\left|\mathscr{S}_{\Gamma}(r, s)\right|$. Therefore, we have from (12.1)

$$
\begin{aligned}
V_{L}(x) & =(-1)^{p} x^{-(p-3 w(\tilde{L})) / 4} P_{\tilde{L}}\left(x^{1 / 2},-\left(x^{1 / 2}+x^{-1 / 2}\right),-\left(x^{1 / 2}+x^{-1 / 2}\right)\right) \\
& =(-1)^{p} x^{-(p-3 w(\tilde{L})) / 4+(v-1) / 2} \sum_{0 \leq r, s<\infty} a_{r, s}\left(-\left(1+x^{-1}\right)\right)^{r}(-(1+x))^{s} .
\end{aligned}
$$

Therefore, it suffices to show that

$$
Q(x)=\sum_{0 \leq r, s<\infty} a_{r, s}\left(-\left(1+x^{-1}\right)\right)^{r}(-(1+x))^{s}
$$

is alternating.

Now, by comparing (12.2) and (12.3), we have $P_{\Gamma}\left(1,-\left(1+x^{-1}\right),-(1+x)\right)=$ $Q(x)$, but since $P_{\Gamma}(1, y-1, z-1)=\chi(\Gamma ; y, z)$ by Proposition 2.5 , it follows that

$$
\chi\left(\Gamma ;-x^{-1},-x\right)=P_{\Gamma}\left(1,-\left(1+x^{-1}\right),-(1+x)\right)=Q(x),
$$

and hence

$$
Q(-x)=\chi\left(\Gamma ; x^{-1}, x\right)
$$

Since Tutte's dichromate $\chi(\Gamma ; y, z)$ is a positive polynomial, all nonzero coefficients of $Q(-x)$ are positive, and hence $Q(x)$ is alternating. It proves Theorem 12.2 . 
Theorem 12.3. Let $L$ be a $\mu$-component link. Then $V_{L}(x)$ can be written as $V_{L}(x)=x^{(\mu-1) / 2} F(x)$, where $F(x)$ is an integer (Laurent) polynomial in $x^{ \pm 1}$.

This theorem has been proved implicitly in [J1]. Since our proof needs lemmas in $\S 13$, the proof will be postponed to Appendix (II).

\section{APPLication to LiNK THEORY (II)-TAit's CONJECTURES}

We have already had two indices $\xi_{\rho}(c)$ and $w(c)$ at each crossing $c$. We now need the third index $\eta_{\rho}(c)$ at $c$.

Let $\tilde{L}$ be an oriented diagram of a link $L$ and $\rho$ shading the of $S^{2}-\tilde{L}$. Let $\eta_{\rho}(c)=w(c) \delta_{\xi_{\rho}(c), w(c)}$, where $\delta$ denotes Kronecker's delta. We define

$$
\eta_{\rho}(\tilde{L})=\sum \eta_{\rho}(c)
$$

where the summation runs over all crossings in $\tilde{L} . \quad \eta_{\rho}(\tilde{L})$ depends not only on the shading $\rho$ but also on the orientation of $\tilde{L}$. Therefore, $\eta_{\rho}(\tilde{L})$ is not determined by the graph $\Gamma(\tilde{L}, \rho)$ alone. To be more precise, let $c_{+}(\tilde{L})$ and $c_{-}(\tilde{L})$ be, respectively, the numbers of crossings in $\tilde{L}$ with positive and negative twists. Note that $w(\tilde{L})=c_{+}(\tilde{L})-c_{-}(\tilde{L})$, and $c_{+}(\tilde{L})+c_{-}(\tilde{L})$ is the total number of crossings in $\tilde{L}$, which will be denoted by $c(\tilde{L})$.

Lemma 13.1. $p(\Gamma(\tilde{L}, \rho))=c_{-}(\tilde{L})+\eta_{\rho}(\tilde{L})$ and $n(\Gamma(\tilde{L}, \rho))=c_{+}(\tilde{L})-\eta_{\rho}(\tilde{L})$.

Proof. There are four cases to be considered. A broken line in Figure 13.1 indicates an edge in the graph of $\tilde{L}$.

From Figure 13.1, we see easily that $p(\Gamma)=\mid\left\{c \in \tilde{L} \mid w(c)=-1, \eta_{\rho}(c)=\right.$ $0\}|+|\left\{c \in \tilde{L} \mid w(c)=1, \eta_{\rho}(c)=1\right\} \mid$, and hence $p(\Gamma)=c_{-}(\tilde{L})+\eta_{\rho}(\tilde{L})$. Similarly, $n(\Gamma)=\left|\left\{c \in \tilde{L} \mid w(c)=1, \eta_{\rho}(c)=0\right\}\right|+\left|\left\{x \in \tilde{L} \mid w(c)=-1, \eta_{\rho}(c)=-1\right\}\right|$, and hence $n(\Gamma)=c_{+}(\tilde{L})-\eta_{\rho}(\tilde{L})$. Q.E.D.

The following lemma is proved in [GL]. See also [BZ].

(1)

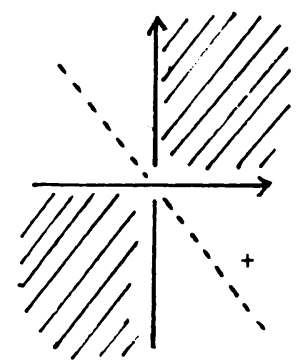

$\mathbf{w}=1$

$\xi=1$

$n=1$
(2)

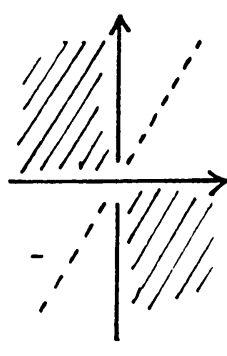

$\mathbf{w}=1$

$\xi=-1$

$n=0$
(3)

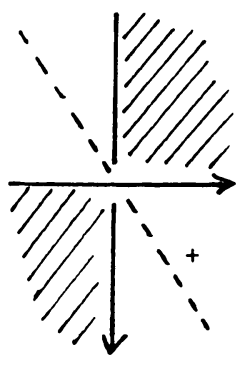

$\mathbf{w}=-1$

$\xi=1$

$\eta=0$
(4)

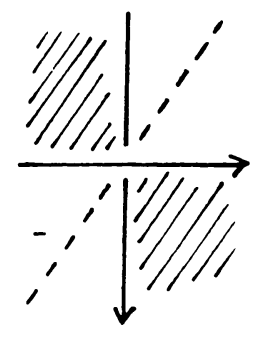

$\mathbf{w}=-1$

$\xi=-1$

$n=-1$

FIGURE 13.1 
Lemma 13.2. $\sigma(L)=\sigma(\Gamma(\tilde{L}, \rho))-\eta_{\rho}(\tilde{L})$.

Remark 13.1. For an alternating link, Lemma 13.2 is an easy consequence of the theorems in [M1]. For a proof of Lemma 13.2, see [GL].

Using these lemmas and Theorem 8.1, we can now prove the following.

Theorem 13.3 (Third Main Theorem). For any link diagram $\tilde{L}$ of a link $L$,

$$
\left\{\begin{array}{l}
d_{\max } V_{L}(x) \leq c_{+}(\tilde{L})-\frac{1}{2} \sigma(L), \\
d_{\min } V_{L}(x) \geq-c_{-}(\tilde{L})-\frac{1}{2} \sigma(L) .
\end{array}\right.
$$

Both equalities hold in (13.1) simultaneously iff $\tilde{L}$ is a reduced alternating diagram or the connected sum of these.

Proof. Let $P$ and $N$ be, as usual, the maximal positive and negative subgraphs of $\Gamma$. Then by Theorems 3.1 and 12.1 we have

$$
\left\{\begin{array}{l}
d_{\max } \mathbf{V}_{L}(x) \leq p(\Gamma)+r_{P}+s_{P}-\frac{p(\Gamma)-n(\Gamma)-3 w(\tilde{L})}{2} \\
d_{\min } \mathbf{V}_{L}(x) \geq-\left(n(\Gamma)+r_{N}+s_{N}\right)-\frac{p(\Gamma)-n(\Gamma)-3 w(\tilde{L})}{2}
\end{array}\right.
$$

Therefore, to prove (13.1), it suffices to show

$$
\begin{aligned}
& \text { (1) } p(\Gamma)+r_{p}+s_{p}-\frac{p(\Gamma)-n(\Gamma)-3 w(\tilde{L})}{2} \leq 2 c_{+}(\tilde{L})-\sigma(L), \\
& \text { (2) }-\left(n(\Gamma)+r_{N}+s_{N}\right)-\frac{p(\Gamma)-n(\Gamma)-3 w(\tilde{L})}{2} \geq-2 c_{-}(\tilde{L})-\sigma(L) .
\end{aligned}
$$

Since $w(\tilde{L})=c_{+}(\tilde{L})-c_{-}(\tilde{L})$ and $p(\Gamma)+n(\Gamma)=c_{+}(\tilde{L})+c_{-}(\tilde{L})$, a straightforward computation shows that (13.3) is equivalent to (13.4):

$$
\begin{array}{ll}
\text { (1) } & \sigma(L) \leq c_{-}(\tilde{L})-\left(r_{P}+s_{P}\right) \\
\text { (2) } & r_{N}+s_{N}-c_{+}(\tilde{L}) \leq \sigma(L) .
\end{array}
$$

However, since $\sigma(L)=\sigma(\Gamma)-\eta_{\rho}(\tilde{L})$ by Lemma 13.2, (13.4) becomes

$$
r_{N}+s_{N}-c_{+}(\tilde{L})+\eta_{\rho}(\tilde{L}) \leq \sigma(\Gamma) \leq c_{-}(\tilde{L})+\eta_{\rho}(\tilde{L})-\left(r_{P}+s_{P}\right)
$$

or, by Lemma 13.1,

$$
r_{N}+s_{N}-n(\Gamma) \leq \sigma(\Gamma) \leq p(\Gamma)-\left(r_{P}+s_{P}\right) .
$$

(13.6) is exactly what we have proved in Theorem 8.1 , and equalities hold in (13.6) simultaneously iff $\tilde{L}$ is a reduced alternating diagram or the connected sum of these.

Theorem 13.3 is now proved.

Remark 13.2. V. F. R. Jones also gives a different estimate for $d_{\max } V_{L}(x)$ for a closed $n$-braid [ $\mathrm{J} 2$, Proposition 14.2]. An alternative proof of this proposition can be found in [M8], where a comparison of these two estimates is briefly discussed. 
Theorem 13.3 yields many important consequences for link diagrams [M3, M4].

Let $L$ be a link. For simplicity, we always assume that $L$ is not split, and hence a diagram $\tilde{L}$ and the graph $\Gamma(\tilde{L})$ are connected.

Let $c(\tilde{L})$ denote the total number of crossings in $\tilde{L}$, i.e., $c(\tilde{L})=c_{+}(\tilde{L})+$ $c_{-}(\tilde{L})$. Moreover, we denote by $c(L)$ the minumum number of crossings any diagram of $L$ can have. Obviously $c(L)$ is a link invariant. A link diagram that has exactly $c(L)$ crossings will be called a minimal diagram.

Now for any diagram $\tilde{L}$ of $L$, we have from (13.1) that

$$
d_{\max } V_{L}(x)-d_{\min } V_{L}(x) \leq c_{+}(\tilde{L})+c_{-}(\tilde{L})=c(\tilde{L}) .
$$

We call $d_{\max } V_{L}(x)-d_{\min } V_{L}(x)$ the span of $V_{L}(x)$ and denote it by span $V_{L}(x) . \quad \operatorname{span} V_{L}(x)$ is a link invariant and (13.7) shows that $\operatorname{span} V_{L}(x)$ gives us a lower bound for the number of crossings any diagram of $L$ can have. This proves the following.

Theorem 13.4. If $L$ is not a split link, then

$$
\operatorname{span} V_{L}(x) \leq c(L)
$$

In particular, if $L$ is alternating and $\tilde{L}$ is a reduced alternating diagram, then equalities in (13.1) give us the following.

Theorem 13.5. If $L$ is a (nonsplit) alternating link, then

$$
\operatorname{span} V_{L}(x)=c(L) \text {. }
$$

Furthermore, for any reduced alternating diagram $\tilde{L}, \operatorname{span} V_{L}(x)=c(\tilde{L})$. Therefore, any reduced alternating diagram is a minimal diagram.

The second statement of Theorem 13.5 implies

Theorem 13.6. span $V_{L}(x)=c(L)$ iff $L$ is a connected sum of alternating links.

Theorems 13.4-13.6 solve some of the long-standing conjectures in knot theory due to P. G. Tait [HKW].

Next, if $L$ is alternating and $\tilde{L}$ is a reduced alternating diagram, then (13.1) yields

$$
d_{\max } V_{L}(x)+d_{\min } V_{L}(x)=c_{+}(\tilde{L})-c_{-}(\tilde{L})-\sigma(L)
$$

and hence

$$
d_{\max } V_{L}(x)+d_{\min } V_{L}(x)=w(\tilde{L})-\sigma(L) .
$$

Since $d_{\max } V_{L}(x), d_{\min } V_{L}(x)$, and $\sigma(L)$ are all link invariants, so is $w(\tilde{L})$. 

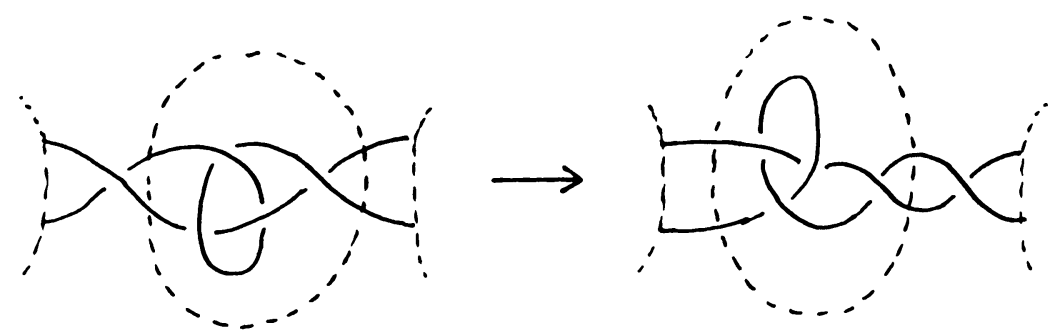

FIGURE 14.1

It also proves another conjecture due to Tait. Namely, we have

Theorem 13.7. If $\tilde{L}_{1}$ and $\tilde{L}_{2}$ are reduced alternating diagrams of the same alternating link, then

$$
w\left(\tilde{L}_{1}\right)=w\left(\tilde{L}_{2}\right) .
$$

In other words, the writhe is independent of the diagram insofar as we consider reduced alternating diagrams.

Remark 13.3. Theorems $13.4-13.7$ are also proved by Thistlethwaite with a slightly different method [Th1]. Kauffman also proved Theorems 13.4-13.6 [K1]. Furthermore, alternative proofs of these theorems may be found in [Tur].

Suppose that an alternating link is amphicheiral; i.e., $L$ and its mirror image $L^{*}$ are of the same type. If $\tilde{L}$ is a reduced alternating diagram of $L$, then the mirror image $\tilde{L}^{*}$ of $\tilde{L}$ is also a reduced alternating diagram of $L^{*}$, and hence, by definition, $w\left(\tilde{L}^{*}\right)=-w(\tilde{L})$. Since $w(\tilde{L})$ is an invariant (Theorem 13.7) and $L$ and $L^{*}$ are of the same type, we should have $w\left(\tilde{L}^{*}\right)=w(\tilde{L})$, which implies that $w(\tilde{L})=0$. In particular, since $c(\tilde{L}) \equiv w(\tilde{L})(\bmod 2)$, we see that $c(\tilde{L})$ is even. This proves the following.

Theorem 13.8. If an alternating link is amphicherial, then for any reduced alternating diagram $\tilde{L}, w(\tilde{L})=0$ and $c(\tilde{L})$ is even.

Corollary 13.9. Let $L$ be an alternating link. Then two of the following three conditions imply the other.

(1) $d_{\max } V_{L}(x)=-d_{\min } V_{L}(x)$,

(2) $\sigma(L)=0$,

(3) $w(\tilde{L})=0$ for any reduced alternating diagram $\tilde{L}$ of $L$.

Proof. A proof follows from (13.9).

\section{APPLICATION TO LINK THEORY (III)-ALTERNATING LINK INVARIANTS}

The last, but unsolved, conjecture of P. G. Tait is stated as follows [HKW].

Conjecture A (P. G. Tait). Reduced alternating diagrams of the same link are "essentially" unique. More precisely, one can pass from one to another by a sequence of "flyping" operations.

A typical flyping operation is shown in Figure 14.1. 


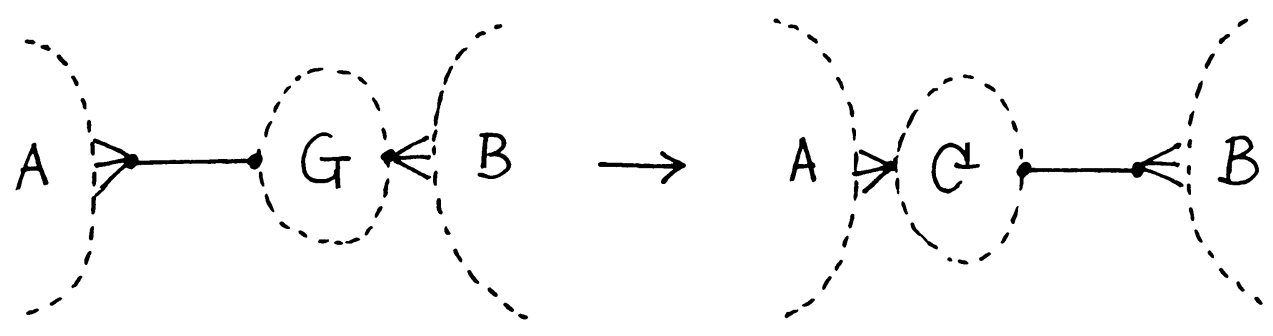

FIGURE 14.2

A flyping on a diagram $\tilde{L}$ translates to an operation $\Omega$ depicted in Figure 14.2 on the graph $\Gamma(\tilde{L})$.

Conjecture A claims roughly that an alternating link type is "essentially" determined by its graph. More precisely, it states that two graphs associated with two reduced alternating diagrams are related by a very restricted type of 2-isomorphism. Therefore, as the first step toward Conjecture A, we propose a much weaker conjecture:

Conjecture $\mathbf{A}^{\prime}$. If $\tilde{L}_{1}$ and $\tilde{L}_{2}$ are reduced alternating diagrams of an alternating link, then $\Gamma\left(\tilde{L}_{1}\right)$ is 2-isomorphic to $\Gamma\left(\tilde{L}_{2}\right)$.

Since two graphs $\Gamma$ and $\Omega(\Gamma)$ have the same polynomial, $P_{\Gamma}(x, y, z)$ would be an invariant of an alternating link if Conjecture $\mathrm{A}^{\prime}$ is true. $\left(P_{\Gamma}(x, y, z)\right.$ is not an invariant for an arbitrary link, however.) Therefore, to disprove Conjecture $\mathrm{A}^{\prime}$, it is enough to show that some invariants derived from $P_{\Gamma}(x, y, z)$ are not invariants for an alternating link. So far, many invariants of the graph associated with a reduced alternating link are, in fact, link invariants.

In this section, we will study and use these invariants of the graph to obtain more information about an alternating link. We assume that the graph associated with an alternating diagram is always positive.

A quantity $\lambda(\tilde{L})$ associated with a link diagram $\tilde{L}$ is called an alternatinglink invariant if $\lambda\left(\tilde{L}_{1}\right)=\lambda\left(\tilde{L}_{2}\right)$ for reduced alternating diagrams $\tilde{L}_{1}$ and $\tilde{L}_{2}$ of the same alternating link.

The following conjecture is weaker then Conjecture $A^{\prime}$, but is still ambitious.

Conjecture B. The polynomial $P_{\Gamma}(x, y, z)$ is an alternating-link invariant.

Whatever Conjecture B may be, some invariants derived from $P_{\Gamma}(x, y, z)$ could be alternating-link invariants. In particular, we propose

Conjecture C. $d_{\max } P_{\Gamma(\tilde{L})}(1, y, z)$ is an alternating-link invariant.

To be more precise, let $L$ be an arbitrary (not necessarily alternating) link and $\tilde{L}$ a diagram of $L$. Let $\Gamma(\tilde{L})$ be the graph of $\tilde{L}$.

Definition 14.1. The chromatic degree $\mathrm{d}^{*}(L)$ of $L$ is defined as

$$
\mathbf{d}^{*}(L)=\min \mathbf{d}(\Gamma(\tilde{L})),
$$


where the minimum is taken over all diagrams $\tilde{L}$ of $L . \mathbf{d}^{*}(L)$ is a link type invariant. If $L$ is an alternating link, then by taking the minimum in (14.1) over all alternating diagrams $\tilde{L}$, we will have the (weak) chromatic degree $\mathbf{d}(L)$ of $L$. Conjecture $C$ implies that $\mathbf{d}(L)=\mathbf{d}(\Gamma(\tilde{L}))$ for any reduced alternating diagram $\tilde{L}$ of $L . \mathbf{d}(L)$ is an alternating-link invariant. Obviously, $\mathbf{d}(L) \geq$ $\mathbf{d}^{*}(L)$, but the reverse inequality seems plausible, and we conjecture that $\mathbf{d}(L)=$ $\mathbf{d}^{*}(L)$ for any alternating link.

Now from Theorems 4.6 and 13.5 , we have immediately

Theorem 14.1. For any (nonsplit) alternating link $L$,

$$
\frac{1}{2}\left[\operatorname{span} V_{L}(x)+1\right] \leq \mathbf{d}(L) \leq \operatorname{span} V_{L}(x)
$$

or equivalently

$$
\operatorname{span} V_{L}(x) \leq 2 \mathbf{d}(L) \leq 2 \operatorname{span} V_{L}(x)
$$

$\mathbf{d}(L)$ is also related to another link invariant, the Alexander polynomial.

A link $L$ can be expressed (not uniquely) as the *-product of special links, $L_{1}, L_{2}, \ldots, L_{q}[\mathrm{M} 1]$, and we write $L=L_{1} * L_{2} * \cdots * L_{q}$. If $L$ is alternating, then all $L_{i}$ are special alternating. Furthermore, if $L$ is fibre, then each $L_{i}$ is fibre and hence $L_{i}$ is an elementary torus link.

Theorem 14.2. Suppose $L=L_{1} * L_{2} * \cdots * L_{q}$. Then

$$
\mathbf{d}^{*}(L) \leq \mathbf{d}^{*}\left(L_{1}\right)+\mathbf{d}^{*}\left(L_{2}\right)+\cdots+\mathbf{d}^{*}\left(L_{q}\right) .
$$

If $L$ is an alternating link, then $\mathbf{d}^{*}\left(L_{i}\right)$ can be replaced by $\mathbf{d}\left(L_{i}\right)$ in (14.2). In particular, if $L$ is alternating and fibred then

$$
\mathbf{d}(L) \leq \operatorname{deg} \Delta_{L}(t),
$$

where $\Delta_{L}(t)$ denotes the reduced Alexander polynomial of $L$ [BZ, p. 131]. Equality holds in (14.3) if $\operatorname{deg} \Delta_{L_{i}}(t)=1$ for all $i$.

Proof. A proof will follow from Theorem 4.5. Suppose that $L=L_{1} * L_{2}$. From the definition of *-product, we see [M1] that $L_{1}$ and $L_{2}$ have link diagrams $\tilde{L}_{1}$ and $\tilde{L}_{2}$ such that a link diagram $\tilde{L}$ is constructed from $\tilde{L}_{i}$ and the graph $\Gamma(\tilde{L})$ has the following properties.

$(14.4)(1) \Gamma(\tilde{L})$ is a union of the graph $\Gamma\left(\tilde{L}_{1}\right)$ associated with $\tilde{L}_{1}$ and some graph $\Gamma_{2}$,

(2) $\Gamma\left(\tilde{L}_{1}\right)$ and $\Gamma_{2}$ meet in a finite number of common vertices, say $v_{1}, v_{2}, \ldots, v_{m}$

(3) the graph $\Gamma\left(\tilde{L}_{2}\right)$ associated with $L_{2}$ is $\Gamma_{2} /\left\{v_{1}, v_{2}, \ldots, v_{m}\right\}$.

Now (4.6) yields

$$
\mathbf{d}^{*}(\Gamma(\tilde{L})) \leq \mathbf{d}^{*}\left(\Gamma\left(\tilde{L}_{1}\right)\right)+\mathbf{d}^{*}\left(\Gamma\left(\tilde{L}_{2}\right)\right)
$$

and hence we have, by definition, $\mathbf{d}^{*}(L) \leq \mathbf{d}^{*}\left(L_{1}\right)+\mathbf{d}^{*}\left(L_{2}\right)$, which proves (14.2).

A similar argument is valid for alternating links. 
Suppose, furthermore, that $L$ is fibred. Then $L_{i}$ is an elementary torus link, and hence the graph $\Gamma\left(\tilde{L}_{i}\right)$ is a $k_{i}$-circuit for some integer $k_{i}$. Since $\mathbf{d}\left(L_{i}\right)=k_{i}-1=\operatorname{deg} \Delta_{L_{i}}(t)$ and $\sum_{i=1}^{q}\left(k_{i}-1\right)=\sum_{i=1}^{q} \operatorname{deg} \Delta_{L_{i}}(t)=\operatorname{deg} \Delta_{L}(t)$ [M1], (14.2) follows immediately.

If $\operatorname{deg} \Delta_{L_{i}}(t)=1$, then

$$
\left|E\left(\Gamma\left(\tilde{L}_{i}\right)\right)\right|=k_{i}=2 \quad \text { and }|E(\Gamma(\tilde{L}))|=\sum_{i=1}^{q}\left|E\left(\Gamma\left(\tilde{L}_{i}\right)\right)\right|=2 q .
$$

Then Theorem 4.6 implies that $\mathbf{d}(\Gamma(\tilde{L})) \leq \sum_{i=1}^{q} \mathrm{~d}\left(L_{i}\right)=q$ and hence equality holds in (14.2).

Remark 14.1. (14.3) is not necessarily true when $L$ is not a fibre knot.

Generally, the determination of $\mathbf{d}(L)$ or $\mathbf{d}^{*}(L)$ is not easy. However, for many knots or links with a small number of crossings, $\mathrm{d}(L)$ can be calculated using Theorem 14.1 and a proposition proved in Appendix (III). For the details, see Appendix (III).

Most of the other simple invariants of $\Gamma(\tilde{L})$ are alternating-link invariants. In fact, we can prove the following proposition.

\section{Proposition 14.3.}

(1) $c_{+}(\tilde{L})$ and $c_{-}(\tilde{L})$ are alternating-link invariants.

(2) $\eta_{\rho}(\tilde{L})$ is an alternating-link invariant.

(3) $|V(\Gamma(\tilde{L}))|,|E(\Gamma(\tilde{L}))|$, and $\beta_{1}(\Gamma(\tilde{L}))$ are alternating-link invariants.

Proof. (1) Since $L$ is an alternating link, it follows from (13.1) that $d_{\max } V_{L}(x)$ $=c_{+}(\tilde{L})-\frac{1}{2} \sigma(L)$, and hence $c_{+}(\tilde{L})$ is an alternating-link invariant. Therefore $c_{-}(\tilde{L})$ is also an alternating-link invariant by Theorem 13.7.

(2) Since $\Gamma(\tilde{L})$ is positive, Lemma 13.1 shows that $c_{+}=\eta_{\rho}(\tilde{L})$, and hence $\eta_{\rho}(\tilde{L})$ is an alternating-link invariant.

(3) Since $\sigma(L)$ is an invariant, it follows from Proposition 7.3 and Lemma 13.2 that $|V(\Gamma)|-1=\sigma(\Gamma)=\sigma(L)+\eta_{\rho}(\tilde{L})$ is an alternating-link invariant, and hence $|V(\Gamma)|$ is an alternating-link invariant. Furthermore, $|E(\Gamma)|=c(\tilde{L})$ is an alternating-link invariant by Theorem 13.5. Finally, since $\Gamma(\tilde{L})$ is planar, $|V(\Gamma(\tilde{L}))|-|E(\Gamma(\tilde{L}))|+\beta_{1}(\Gamma(\tilde{L}))+1=2$, and hence $\beta_{1}(\Gamma(\tilde{L}))$ is an alternatinglink invariant. Q.E.D.

Now since $p=|E(\Gamma(\tilde{L}))|, w(\tilde{L})$, and $|V(\Gamma(\tilde{L}))|$ are alternating-link invariants, it follows from (12.1) and (12.2) that

$$
Q(x)=\sum_{r, s}\left|\mathscr{S}_{\Gamma}(r, s)\right|\left(-\left(1+x^{-1}\right)\right)^{r}(-(1+x))^{s}
$$

is an alternating-link invariant. Since $P(1, y, z)=\sum_{r, s}\left|\mathscr{S}_{\Gamma}(r, s)\right| y^{r} z^{s}$, at least we can show that $P\left(1,-\left(1+x^{-1}\right),-(1+x)\right)$ is an alternating-link invariant. We can prove, moreover, the following. 
Proposition 14.4. $P\left(1, t^{-1}, t\right)=\sum_{r, s}\left|\mathscr{S}_{\Gamma}(r, s)\right| t^{-r+s}$ is an alternating-link invariant.

Proof. Let $G \in \mathscr{S}_{\Gamma}(r, s)$. Denote $v=|V(\Gamma)|=|V(G)|$ and $m=|E(\Gamma)|$. Then $r-s=v-1-|E(G)|$, and hence $|E(G)|=s-r+v-1$. Therefore, we have

$$
\begin{gathered}
\sum_{s=r=k}\left|\mathscr{S}_{\Gamma}(r, s)\right|=\left(\begin{array}{c}
m \\
k+v-1
\end{array}\right) \text { and } \\
P\left(1, t^{-1}, t\right)=\sum_{k} \sum_{s-r=k}\left|\mathscr{S}_{\Gamma}(r, s)\right| t^{k}=\sum_{k=-(v-1)}^{m-v+1}\left(\begin{array}{c}
m \\
k+v-1
\end{array}\right) t^{k} .
\end{gathered}
$$

Since $m$ is an alternating-link invariant, so is $P\left(1, t^{-1}, t\right)$. Q.E.D.

It is quite likely that $P(1, t, t)$ is also an alternating-link invariant, and Conjecture $C$ claims that at least $\operatorname{deg} P(1, t, t)$ is an alternating-link invariant. Recently, however, Thistlethwaite proved that $P_{\Gamma}(1, t,-1)$ and $P_{\Gamma}(1,-1, t)$ are both alternating-link invariants [Th3].

Remark 14.2. A property that $\Gamma$ is simple or is of even valency is an alternatinglink invariant. For a proof, see [M9].

If the graph $\Gamma$ of a reduced alternating link diagram $\tilde{L}$ is self-dual, i.e., $\Gamma(\tilde{L}) \approx \Gamma(\tilde{L})^{d}$, then $L$ is amphicheiral. It has been conjectured by Kauffman [K2] that the converse will be true. Although there are many necessary (algebraic) conditions for $L$ to be amphicheiral, none of them is sufficient. For example, it is known [ $\mathrm{J} 1]$ that if a link is amphicheiral, then $V_{L}(x)$ is symmetric, i.e., $V_{L}(x)=V_{L}\left(x^{-1}\right)$, but the converse need not be true even for alternating links. However, for an alternating link the symmetric property of $V_{L}(x)$ seems close to the amphicheirality of $L$ from an algebraic viewpoint. We have strong evidence to support the following conjecture.

Conjecture D. Let $L$ be an alternating link. If $V_{L}(x)$ is symmetric, then $\sigma(L)=$ 0 , and hence $w(\tilde{L})=0$ for any reduced alternating diagram $\tilde{L}$.

Remark 14.3. Conjecture $D$ is true for an alternating closed 3-braid, but it need not be true if symmetry is replaced by the condition $d_{\max } V_{L}(x)=-d_{\min } V_{L}(x)$, or if $L$ is not alternating. In fact, for an alternating knot $K=10_{4}$ in the table in [R], $V_{K}(x)$ is not symmetric, but $d_{\max } V_{K}(x)=-d_{\min } V_{K}(x)$ and $\sigma(K)=2$. Moreover, a nonalternating knot $9_{42}$ has the symmetric Jones polynomial, but $\sigma(K)=2$.

In the theorem below, we prove Conjecture D for some special type of alternating links.

Theorem 14.5. Let $L$ be an alternating link. Let $\tilde{L}$ be a reduced alternating diagram and $\Gamma$ be the graph of $L$. Suppose that $V_{L}(x)$ is symmetric. If both $\Gamma$ and the $\Gamma^{d}$ are simple, then $\sigma(L)=0$.

Proof. Since $\sigma(L)=0$ iff $w(\tilde{L})=0$ by Corollary 13.9 , we will prove that $w(\tilde{L})=0$. Denote $v=|V(\Gamma)|$ and $v^{*}=\left|V\left(\Gamma^{d}\right)\right|$. 
Now a proof will be given by evaluating the first and last few terms of $V_{L}(x)$. Since $\Gamma(\tilde{L})$ is positive, it follows from (2.3), (3.2), and (12.1) that

$$
\begin{aligned}
& d_{\max } V_{L}(x)=\frac{v-1}{2}-\frac{p(\Gamma)-3 w(\tilde{L})}{4}+s_{P} \text { and } \\
& d_{\min } V_{L}(x)=\frac{v^{*}-1}{2}-\frac{p(\Gamma)-3 w(\tilde{L})}{4}-r_{N} .
\end{aligned}
$$

Since $s_{P}=v^{*}-1$ and $r_{N}=v-1$, the symmetric property of $V_{L}(x)$ implies that $v^{*}=v-3 w(\tilde{L})$. Therefore, it suffices to show that $v^{*}=v$.

Now consider the polynomial

$$
Q(x)=\sum_{G \subset \Gamma}(-1)^{r_{G}+s_{G}}\left(1+x^{-1}\right)^{r_{G}}(1+x)^{s_{G}},
$$

where the summation runs over all spanning subgraphs $G$ in $\Gamma$. Since $\Gamma$ is positive, we can write $Q(x)$ as

$$
Q(x)=\sum_{\substack{0 \leq r \leq v-1 \\ 0 \leq s \leq v^{*}-1}}(-1)^{r+s}\left|\mathscr{S}_{\Gamma}(r, s)\right|\left(1+x^{-1}\right)^{r}(1+x)^{s} .
$$

(See (2.3) and (12.3).) It is easy to see that $d_{\max } Q(x)=v^{*}-1$ and $d_{\min } Q(x)=$ $-(v-1)$. Furthermore, the terms with these extreme degrees have coefficients $(-1)^{v-1}$ and $(-1)^{v^{*}-1}$. (See Proposition 6.1.) Therefore, $v \equiv v^{*}(\bmod 2)$. Next, we consider the terms of degrees $v^{*}-2$ and $-(v-2)$. They are given in the following.

Lemma 14.6. Let $\alpha$ and $\beta$ be, respectively, the coefficients of the terms in $Q(x)$ of degrees $v^{*}-2$ and $-(v-2)$. Then

$$
(-1)^{v^{*}-1} \alpha=\left(\begin{array}{c}
v^{*}-1 \\
1
\end{array}\right)\left|\mathscr{S}_{\Gamma}\left(0, v^{*}-1\right)\right|+\sum_{i=0}^{v-1}(-1)^{i}\left|\mathscr{S}_{\Gamma}\left(i, v^{*}-2\right)\right|
$$

and

$$
(-1)^{v-1} \beta=\left(\begin{array}{c}
v-1 \\
1
\end{array}\right)\left|\mathscr{S}_{\Gamma}(v-1,0)\right|+\sum_{j=0}^{v^{*}-1}(-1)^{j}\left|\mathscr{S}_{\Gamma}(v-2, j)\right| .
$$

A proof is straightforward.

We now apply Proposition 6.1 and 6.2. Since $\Gamma$ and $\Gamma^{d}$ are simple, most of the terms in $\alpha$ and $\beta$ vanish except for $\left|\mathscr{S}_{\Gamma}(v-1,0)\right|=1,\left|\mathscr{S}_{\Gamma}(v-2,0)\right|=$ $|E(\Gamma)|=p(\Gamma),\left|\mathscr{S}_{\Gamma}\left(0, v^{*}-1\right)\right|=1$, and $\left|\mathscr{S}_{\Gamma}\left(0, v^{*}-2\right)\right|=|E(\Gamma)|=p(\Gamma)$. Since $\Gamma^{d}$ is simple, $\Gamma$ has no twigs and $\beta_{1}(\Gamma)=v^{*}-1$. Therefore, $\alpha=\beta$ yields $v=v^{*}$ as needed. This proves the theorem.

\section{APPLiCATION TO LINK THEORY (IV)-NONALTERNATING LINKS}

If $L$ is not alternating, most of the theorems in the previous sections are no longer valid. As pointed out by K. Perko, there are two minimal nonalternating 
diagrams of the same 10-crossing knot $\left(10_{161}=10_{162}\right.$ in [R] that have distinct writhes, one of which is 10 and the other is 8 . Therefore $w(\tilde{L})$ is no longer an invariant of a knot type. Nevertheless, the writhes of two link diagrams with the same number of crossings cannot be so far off.

Proposition 15.1. Let $\tilde{L}_{1}$ and $\tilde{L}_{2}$ be two link diagrams of a link $L$ such that $c\left(\tilde{L}_{1}\right)=c\left(\tilde{L}_{2}\right)$. Let $k=c\left(\tilde{L}_{1}\right)-\operatorname{span} V_{L}(x)$. Then

$$
\left|w\left(\tilde{L}_{1}\right)-w\left(\tilde{L}_{2}\right)\right| \leq 2 k .
$$

Proof. Let $\alpha_{L}=d_{\max } V_{L}(x)+\frac{1}{2} \sigma(L)$ and $\beta_{L}=d_{\min } V_{L}(x)+\frac{1}{2} \sigma(L)$. Since $\alpha_{L} \leq c_{+}\left(\tilde{L}_{i}\right)$ and $\beta_{L} \geq-c_{-}\left(\tilde{L}_{i}\right), i=1,2$, by Theorem 13.3 we can write $c_{+}\left(\tilde{L}_{i}\right)=\alpha_{L}+\lambda_{i}$ and $c_{-}\left(\tilde{L}_{i}\right)=-\beta_{L}+\mu_{i}$, where $\lambda_{i}, \mu_{i} \geq 0$. Then $c\left(\tilde{L}_{i}\right)=$ $c_{+}\left(\tilde{L}_{i}\right)+c_{-}\left(\tilde{L}_{i}\right)=\alpha_{L}-\beta_{L}+\lambda_{i}+\mu_{i}=\operatorname{span} V_{L}(x)+\lambda_{i}+\mu_{i}$, and hence $\lambda_{i}+\mu_{i}=k$. Therefore,

$$
\begin{aligned}
\left|w\left(\tilde{L}_{1}\right)-w\left(\tilde{L}_{2}\right)\right| & =\left|\left(c_{+}\left(\tilde{L}_{1}\right)-c_{-}\left(\tilde{L}_{1}\right)\right)-\left(c_{+}\left(\tilde{L}_{2}\right)-c_{-}\left(\tilde{L}_{2}\right)\right)\right| \\
& =\left|\left(\alpha_{L}+\lambda_{1}+\beta_{L}-\mu_{1}\right)-\left(\alpha_{L}+\lambda_{2}+\beta_{L}-\mu_{2}\right)\right| \\
& =\left|\lambda_{1}-\mu_{1}-\lambda_{2}+\mu_{2}\right| \\
& \leq \lambda_{1}+\mu_{1}+\lambda_{2}+\mu_{2}=2 k .
\end{aligned}
$$

Corollary 15.2. If $L$ is amphicheiral, then $|w(\tilde{L})| \leq k$.

Proof. Since $L$ is amphicheiral, $V_{L}(x)=V_{L}\left(x^{-1}\right)$ and hence $d_{\max } V_{L}(x)=$ $-d_{\min } V_{L}(x)$. Also $\sigma(L)=0$, and therefore $\alpha_{L}=d_{\max } V_{L}(x)$ and $\beta_{L}=$ $d_{\min } V_{L}(x)=-\alpha_{L}$. Then $|w(\tilde{L})|=\left|c_{+}(\tilde{L})-c_{-}(\tilde{L})\right|=\mid d_{\max } V_{L}(x)+d_{\min } V_{L}(x)+$ $\lambda-\mu|=| \lambda-\mu \mid \leq \lambda+\mu=k$, Q.E.D.

Proposition 15.1 can be slightly improved if both $\alpha_{L}$ and $\beta_{L}$ are nonnegative (or both nonpositive).

Suppose that $\alpha_{L}$ and $\beta_{L}$ are nonnegative. Since $c_{-}\left(\tilde{L}_{i}\right) \geq 0$, we see that $\mu_{i} \geq \beta_{L} \geq 0$. Let $\mu_{i}-\beta_{L}=\gamma_{i} \geq 0$. Then $\lambda_{i}+\gamma_{i}=\lambda_{i}+\mu_{i}-\beta_{L}=k-\beta_{L} \leq k$. Therefore, $\left|w\left(\tilde{L}_{1}\right)-w\left(\tilde{L}_{2}\right)\right|=\left|\lambda_{1}-\mu_{1}-\lambda_{2}+\mu_{2}\right|=\left|\lambda_{1}-\lambda_{2}-\left(\mu_{1}-\beta_{L}\right)+\left(\mu_{2}-\beta_{L}\right)\right|=$ $\left|\lambda_{1}-\gamma_{1}-\lambda_{2}+\gamma_{2}\right| \leq \lambda_{1}+\gamma_{1}+\lambda_{2}+\gamma_{2}=2\left(k-\beta_{L}\right)$. This proves the following.

Proposition 15.3. If both $\alpha_{L}$ and $\beta_{L}$ are nonnegative (or nonpositive), then

$$
\left|w\left(\tilde{L}_{1}\right)-w\left(\tilde{L}_{2}\right)\right| \leq 2\left(k-\beta_{L}\right), \quad\left(\text { or }\left|w\left(\tilde{L}_{1}\right)-w\left(\tilde{L}_{2}\right)\right| \leq 2\left(k+\alpha_{L}\right)\right) .
$$

The knot $K=10_{161}$ has two 10-crossing diagrams $\tilde{K}_{1}$ and $\tilde{K}_{2}$ with $w\left(\tilde{K}_{1}\right)$ $=10$ and $w\left(\tilde{K}_{2}\right)=8$. Since $V_{k}(x)=x^{3}+x^{6}-x^{7}+x^{8}-x^{9}+x^{10}-x^{11}$ and $\sigma(K)=-4$, it follows that $\alpha_{K}=9$ and $\beta_{K}=1$. Furthermore, since span $V_{K}(x)=8, k=2$ and $k-\beta_{K}=1$. Therefore, for any 10-crossing diagram $\tilde{K}$ of $K,\left|w(\tilde{K})-w\left(\tilde{K}_{1}\right)\right| \leq 2$. Since $\tilde{K}_{1}$ has the writhe 10, Proposition 15.3 shows that the writhe of any 10-crossing diagram of $K$ must be either 8 or 10 . In particular, $K$ cannot have a diagram with writhe -10 .

A link is called a positive link if $L$ has a link diagram $\tilde{L}$ without negative crossings, i.e., $c_{-}(\tilde{L})=0$, and hence $c_{+}(\tilde{L})=c(\tilde{L})$. A negative link is a link 
that has a diagram $\tilde{L}$ with $c_{+}(\tilde{L})=0$. A typical example of a positive (or negative) link is a closed positive (or negative) braid. The knot $10_{161}$ is a positive knot. We should note that a positive (or negative) link diagram need not be a minimal diagram.

Proposition 15.4. Let $L$ be a positive (or negative) link with $\operatorname{span} V_{L}(x)>0$. Then $L$ cannot be a negative (or positive) link.

Proof. Suppose that $L$ has a positive diagram $\tilde{L}_{1}$ and a negative diagram $\tilde{L}_{2}$, and hence $c_{-}\left(\tilde{L}_{1}\right)=0$ and $c_{+}\left(\tilde{L}_{2}\right)=0$. Then it follows from (13.1) that

$$
\begin{aligned}
& d_{\max } V_{L}(x) \leq c_{+}\left(\tilde{L}_{2}\right)-\frac{1}{2} \sigma(L)=-\frac{1}{2} \sigma(L) \text { and } \\
& d_{\min } V_{L}(x) \geq-c_{-}\left(\tilde{L}_{1}\right)-\frac{1}{2} \sigma(L)=-\frac{1}{2} \sigma(L),
\end{aligned}
$$

and hence $d_{\max } V_{L}(x)=d_{\min } V_{L}(x)=-\frac{1}{2} \sigma(L)$, which yields $\operatorname{span} V_{L}(x)=0$, a contradiction.

Remark 15.1. Recently, P. Traczyk proved that the condition $\operatorname{span} V_{L}(x)>0$ in Proposition 15.4 can be replaced by the condition that $L$ be knotted [Tr2]. Proposition 15.4 is already known for closed positive braids. In fact, the main theorem of $\mathrm{L}$. Rudolph $[\mathrm{Ru}]$ implies that a closed positive braid cannot be represented as a closed negative braid unless it is unknotted.

\section{APPENDIX (I)—Proofs OF TheOREMS 9.5 AND 9.6}

Given a planar graph $\Gamma$, we can construct uniquely a link diagram $(\tilde{L}, \rho)$ of a link $L$ with shading $\rho$. If we change $\rho$ to the opposite $\rho^{\prime}$, we obtain the opposite graph of the dual of $\Gamma$, which we denote by $\hat{\Gamma}$. Therefore $B_{\hat{\Gamma}}=-B_{\Gamma^{d}}$. Proof of Theorem 9.5. Let $\Gamma$ be the graph associated with a link diagram $\tilde{L} . B_{\Gamma}$ coincides with the so-called Goeritz matrix of $L$. (See [BZ].) It is well known that $B_{\Gamma}(i)$ (for any $i$ ) represents the relation matrix of $H_{1}\left(M_{2}(L) ; \mathbf{Z}\right)$, where $M_{2}(L)$ is the branched double cover of $L$ in $S^{3}$. $\left(M_{2}(L)\right.$ does not depend on an orientation of $L$.) Therefore $\operatorname{null}\left(B_{\Gamma}(i)\right)$ is exactly the first Betti number of $M_{2}(L)$, i.e., $\operatorname{null}\left(B_{\Gamma}(i)\right)=\operatorname{dim}_{\mathbf{Q}} H_{1}\left(M_{2}(L) ; \mathbf{Q}\right)$. Since $B_{\hat{\Gamma}}$ is also the Goeritz matrix of the same link $L$, we have again null $B_{\hat{\Gamma}}(i)=\operatorname{dim}_{\mathbf{Q}} H_{1}\left(M_{2}(L) ; \mathbf{Q}\right)$. This proves Theorem 9.5.

Proof of Theorem 9.6. Lemma 13.2 shows that $\sigma(L)$ can be calculated by using any Goeritz matrix of $L$. We use two Goeritz matrices $B_{\Gamma}(i)$ and $B_{\hat{\Gamma}}(i)$. Since $B_{\hat{\Gamma}^{\prime}}(i)=-B_{\Gamma^{d}}(i)$, it follows that $\sigma(\hat{\Gamma})=\sigma\left(B_{\hat{\Gamma}^{\prime}}(i)\right)=-\sigma\left(B_{\Gamma^{d}}(i)\right)=-\sigma\left(\Gamma^{d}\right)$.

Now let $\rho$ and $\rho^{\prime}$ be opposite shadings of $S^{2}-\tilde{L}$, and assume that $\Gamma=$ $\Gamma(\tilde{L}, \rho)$. Then $\hat{\Gamma}=\Gamma\left(\tilde{L}, \rho^{\prime}\right)$. Lemma 13.2 now gives us

(1) $\sigma(L)=\sigma(\Gamma)-\eta_{\rho}(\tilde{L})$,

(2) $\sigma(L)=\sigma(\hat{\Gamma})-\eta_{\rho^{\prime}}(\tilde{L})$.

Since $\sigma(\hat{\Gamma})=-\sigma\left(\Gamma^{d}\right)$, we have from (A.1)

$$
\sigma(\Gamma)-\eta_{\rho}(\tilde{L})=-\sigma\left(\Gamma^{d}\right)-\eta_{\rho^{\prime}}(\tilde{L})
$$


and hence

$$
\sigma(\Gamma)+\sigma\left(\Gamma^{d}\right)=\eta_{\rho}(\tilde{L})-\eta_{\rho^{\prime}}(\tilde{L}) .
$$

We calculate $\eta_{\rho}$ and $\eta_{\rho^{\prime}}$. From Figure 13.1, we see immediately that

$$
\begin{aligned}
\eta_{\rho}(\tilde{L})= & \mid\left\{c \in \tilde{L} \mid w(c)=1 \text { and } \xi_{\rho}(c)=1\right\} \mid \\
& -\mid\left\{c \in \tilde{L} \mid w(c)=-1 \text { and } \xi_{\rho}(c)=-1\right\} \mid,
\end{aligned}
$$

while

$$
\begin{aligned}
\eta_{\rho^{\prime}}(\tilde{L})= & \mid\left\{c \in \tilde{L} \mid w(c)=1 \text { and } \xi_{\rho^{\prime}}(c)=1\right\} \mid \\
& -\mid\left\{c \in \tilde{L} \mid w(c)=-1 \text { and } \xi_{\rho^{\prime}}(c)=-1\right\} \mid \\
= & \mid\left\{c \in \tilde{L} \mid w(c)=1 \text { and } \xi_{\rho}(c)=-1\right\} \mid \\
& -\mid\left\{c \in \tilde{L} \mid w(c)=-1 \text { and } \xi_{\rho^{\prime}}(c)=1\right\} \mid,
\end{aligned}
$$

because $\xi_{\rho^{\prime}}(c)=-\xi_{\rho}(c)$. Therefore,

$$
\begin{aligned}
\eta_{\rho}(\tilde{L})-\eta_{\rho^{\prime}}(\tilde{L}) & =\left|\left\{c \in \tilde{L} \mid \xi_{\rho}(c)=1\right\}\right|-\left|\left\{c \in \tilde{L} \mid \xi_{\rho}(c)=-1\right\}\right| \\
& =p(\Gamma)-n(\Gamma) .
\end{aligned}
$$

This proves Theorem 9.6.

APPENDIX (II)-A PROOF OF THEOREM 12.3

For a spanning subgraph $G$ of $\Gamma$, we see from (6.1) that $p(G)-n(G)=$ $-2 n(G)+p(G)+n(G)=-2 n(G)+(v-1)-r_{G}+s_{G}$, and hence

$$
x^{p(G)-n(G)} y^{r_{G}} z^{s_{G}}=x^{-2 n(G)+v-1}\left(\frac{y}{x}\right)^{r_{G}}(x z)^{s_{G}},
$$

where $v=|V(\Gamma)|$. Therefore, we can write

$$
P_{\Gamma}(x, y, z)=x^{v-1} \sum_{G} x^{-2 n(G)}\left(\frac{y}{x}\right)^{r_{G}}(x z)^{s_{G}} .
$$

Then

$$
\begin{aligned}
P_{\Gamma}\left(x^{1 / 2},\right. & \left.-\left(x^{1 / 2}+x^{-1 / 2}\right),-\left(x^{1 / 2}+x^{-1 / 2}\right)\right) \\
= & x^{\frac{1-1}{2}} \sum_{G} x^{-n(G)}\left(-\left(1+x^{-1}\right)\right)^{r_{G}}(-(1+x))^{s_{G}},
\end{aligned}
$$

and hence

$$
V_{L}(x)=(-1)^{p-n} x^{-\frac{p-n-3 w(L)}{4}+\frac{r-1}{2}} \sum_{G} x^{-n(G)}\left(-\left(1+x^{-1}\right)\right)^{r_{G}}(-(1+x))^{s_{G}} .
$$

Therefore, it is sufficient to prove that

$$
-\frac{p-n-3 w(\tilde{L})}{4}+\frac{v-1}{2} \equiv \frac{\mu-1}{2}(\bmod 1)
$$

or equivalently

$$
-\frac{p-n-3 w(\tilde{L})}{2}+v-1 \equiv \mu-1 \quad(\bmod 2)
$$


Now, since $p+n=c_{+}(\tilde{L})+c_{-}(\tilde{L})$ and $w(\tilde{L})=c_{+}(\tilde{L})-c_{-}(\tilde{L})$, a straightforward computation shows that

$$
-\frac{p-n-3 w(\tilde{L})}{2}+v-1 \equiv c_{+}(\tilde{L})+n+v-1 \quad(\bmod 2) .
$$

Since $c_{+}(\tilde{L})-n=\eta_{\rho}(\tilde{L})\left(\right.$ Lemma 13.1) and $\eta_{\rho}(\tilde{L})=\sigma(\Gamma)-\sigma(L)$ (Lemma 13.2), it is enough to show that

$$
v-1+\sigma(\Gamma)-\sigma(L) \equiv \mu-1 \quad(\bmod 2) .
$$

Now, since $B_{\Gamma}$ is a $(v-1) \times(v-1)$ matrix, we see that $\sigma(\Gamma) \equiv(v-1)-$ $\operatorname{null}\left(B_{\Gamma}(i)\right)(\bmod 2)$. On the other hand, $\operatorname{null}\left(B_{\Gamma}(i)\right)$ is equal to the first Betti number of the branched double cover $M_{2}(L)$ of $L$ in $S^{3}$ and it is known [M1] that $\sigma(L) \equiv \mu-1-\beta_{1}\left(M_{2}(L)\right)(\bmod 2)$. Therefore,

$$
\begin{aligned}
\sigma(\Gamma) & \equiv v-1-\operatorname{null}\left(B_{\Gamma}(i)\right) \equiv v-1-\beta_{1}\left(M_{2}(L)\right) \\
& \equiv v-1+\sigma(L)-(\mu-1) \quad(\bmod 2),
\end{aligned}
$$

which proves (A.3) and Theorem 12.3.

\section{APPENDIX (III)-DETERMINATION OF CHROMATIC DEGREE OF A LINK}

Let $L$ be a (nonsplit) prime alternating link and $\tilde{L}$ a reduced alternating diagram of $L$. Let $\Gamma(\tilde{L})$ be the graph of $\tilde{L}$. Then we have

\section{Proposition A.1.}

(1) If $\mathbf{d}(\Gamma(\tilde{L}))=\frac{1}{2}[|E(\Gamma(\tilde{L}))|+1]$, then $\mathbf{d}^{*}(L)=\mathbf{d}(L)=\frac{1}{2}[|E(\Gamma(\tilde{L}))|+1]=$ $\frac{1}{2}\left[\operatorname{span} V_{L}(x)+1\right]$.

(2) If $\mathbf{d}(L)=\frac{1}{2}[|E(\Gamma(\tilde{L}))|]+1$, then $\mathbf{d}^{*}(L)=\mathbf{d}(L)$.

(3) If $\mathbf{d}(\Gamma(\tilde{L}))=\max \left\{d\left(V_{\Gamma(\tilde{L})}\right), d(\Gamma(\tilde{L}))\right\}$, then $\mathbf{d}(L)=\mathbf{d}(\Gamma(\tilde{L}))$.

Proof. (1) It follows easily from Theorem 4.6 and Theorem 13.5 that $\mathrm{d}(L)=$ $\frac{1}{2}[|E(\Gamma(\tilde{L}))|+1]$. Let $\tilde{L}_{0}$ be any nonalternating diagram of $L$. Then by Theorem 13.3, we have $c\left(\tilde{L}_{0}\right) \supsetneqq c(\tilde{L})$, and hence $\left|E\left(\Gamma\left(\tilde{L}_{0}\right)\right)\right| \supsetneqq|E(\Gamma(\tilde{L}))|$. Therefore $\mathbf{d}\left(\Gamma\left(\tilde{L}_{0}\right)\right) \geq \frac{1}{2}\left[\left|E\left(\Gamma\left(\tilde{L}_{0}\right)\right)\right|+1\right] \geq \frac{1}{2}[|E(\Gamma(\tilde{L}))|+1]=\mathbf{d}(\Gamma(\tilde{L}))=\mathbf{d}(L)$, which yields $\mathbf{d}^{*}(L)=\mathbf{d}(L)$.

(2) We have shown in (1) that for any nonalternating diagram $\tilde{L}_{0}, \mathbf{d}\left(\Gamma\left(L_{0}\right)\right)$ $\geq \frac{1}{2}\left[\left|E\left(\Gamma\left(\tilde{L}_{0}\right)\right)\right|+1\right] \geq \frac{1}{2}[|E(\Gamma(\tilde{L}))|+2]=\frac{1}{2}[|E(\Gamma(\tilde{L}))|]+1$, and hence, if $\mathbf{d}(L)$ $=\frac{1}{2}[|E(\Gamma(L))|]+1$, then $\mathbf{d}\left(\Gamma\left(L_{0}\right)\right) \geq \mathbf{d}(L)$ and $\mathbf{d}^{*}(L)=\mathbf{d}(L)$.

(3) Since $|V(\Gamma(\tilde{L}))|$ and $d(\Gamma(\tilde{L}))=\beta_{1}(\Gamma(\tilde{L}))+1$ are alternating-link invariants, it follows that for any reduced alternating diagram $\tilde{L}_{1},\left|V\left(\Gamma\left(\tilde{L}_{1}\right)\right)\right|=$ $|V(\Gamma(\tilde{L}))|$ and $d\left(\Gamma\left(\tilde{L}_{1}\right)\right)=d(\Gamma(\tilde{L}))$, and hence

$$
\mathbf{d}\left(\Gamma\left(L_{1}\right)\right) \geq \max \left\{d\left(V_{\Gamma\left(L_{1}\right)}\right), d\left(\Gamma\left(L_{1}\right)\right)\right\}=\max \left\{d\left(V_{\Gamma(L)}\right), d(\Gamma(L))\right\}=\mathbf{d}(L) .
$$

Therefore, $\mathbf{d}^{*}(L)=\mathbf{d}(L)$. Q.E.D.

Using Proposition A.1, Theorem 14.2, and some theorems on knots or links [M1], we can determine the chromatic degree of each of 220 knots or links out of 380 knots or links with up to 10 crossings in the table in [Ro]. 


$$
\text { APPENDIX (IV) }-f(\Gamma ; t, x, y) \text { AND } P_{\Gamma}(x, y, z)
$$

Let $\Gamma$ be a positive connected graph. Denote $v=|V(\Gamma)|$ and $p=|E(\Gamma)|$. The polynomial $f(\Gamma ; t, x, y)$ defined by $\mathrm{S}$. Negami is given by

$$
f(\Gamma ; t, x, y)=\sum_{i=0}^{p} \sum_{j=1}^{v} b_{i j} t^{j} x^{p-i} y^{i},
$$

where $b_{i j}$ is the number of subsets $Y$ in $E(\Gamma)$ such that $|E(Y)|=i$ and $\beta_{0}(\Gamma-Y)=j$. Since $\Gamma-Y$ is a spanning subgraph of $\Gamma, b_{i j}$ is exactly the number of spanning subgraphs $G$ such that $\beta_{0}(G)=j$ and $|E(G)|=p-i$. Let $G \in \mathscr{S}_{\Gamma}(r, s)$. Then (6.1) shows that $|E(G)|=v-r+s-1$, and hence $\left|\mathscr{S}_{\Gamma}(r, s)\right|=b_{p-v+r-s+1, r+1}$. Let $b=\beta_{1}(\Gamma)$. Since $\Gamma$ is connected, we see that $b=p-v+1$. Now using Proposition 2.6, we obtain

$$
\begin{aligned}
P_{\Gamma}(x, y, z) & =\sum_{r, s}\left|\mathscr{S}_{\Gamma}(r, s)\right| x^{v-r+s-1} y^{r} z^{s} \\
& =\sum_{r, s} b_{p-v-s+r+1, r+1} x^{v-r+s-1} y^{r} z^{s} \\
& =y^{-1} \sum_{r, s} b_{p-v-s+r+1, r+1} x^{v-r+s-1} y^{r+1} z^{s} \\
& =y^{-1} \sum_{i, j} b_{i, j} x^{p-i} y^{j} z^{b-i+j-1} \\
& =y^{-1} z^{b-1} \sum_{i, j} b_{i, j} x^{p-i}(y z)^{j} z^{-i} \\
& =y^{-1} z^{b-1} f\left(\Gamma ; y z, x, z^{-1}\right) .
\end{aligned}
$$

This proves the following.

Proposition A.2. $P_{\Gamma}(x, y, z)=y^{-1} z^{b-1} f\left(\Gamma ; y z, x, z^{-1}\right)$ and conversely,

$$
f(\Gamma ; t, x, y)=t y^{b} P_{\Gamma}\left(x, t y, y^{-1}\right),
$$

where $b=\beta_{1}(\Gamma)$.

The following splitting formula for $P_{\Gamma}(x, y, z)$ is an immediate consequence of that for $f(\Gamma ; t, x, y)$.

Proposition A.3. Using the notation in [N, Theorem 4.4],

$$
y^{-1} P_{\Gamma}(x, y, z)=\sum_{\gamma, \delta} P_{H / \gamma}(x, y, z) B_{n}(\gamma, \delta ; y z) z^{|\gamma|+|\delta|-n} P_{K / \delta}(x, y, z) .
$$

\section{REFERENCES}

[Be] C. Berge, Graphs and hypergraphs, North-Holland, Amsterdam, 1973.

[Bi] N. Biggs, Algebraic graph theory, Cambridge Univ. Press, 1974.

[BC] M. Behzad and C. Chartrand, Introduction to the theory of graph, Allyn \& Bacon, Boston, Mass., 1971. 
[BZ] G. Burde and H. Zieschang, Knots, De Gruyter, Berlin, 1985.

[GL] C. McA. Gordon and R. A. Litherland, On the signature of a link, Invent. Math. 47 (1978), 53-69.

[GLM] C. MaA. Gordon, R. A. Litherland, and K. Murasugi, Signatures of covering links, Canad. J. Math. 33 (1981), 381-394.

[HKW] P. de la Harpe, M. Kervaire, and C. Weber, On the Jones polynomial, Enseign. Math. 32 (1986), 271-335.

[J1] V. F. R. Jones, A polynomial invariant for knots via von Neumann algebras, Bull. Amer. Math. Soc. 89 (1985), 103-111.

[J2] _ Hecke algebra representations of braid groups and link polynomials, Ann. of Math. 126 (1987), 335-388.

[K1] L. H. Kauffman, State models and the Jones polynomial, Topology 26 (1987), 395-407.

[K2] __, New invariants in the theory of knots, Amer. Math. Monthly 95 (1988), 195-242.

[M1] _ , On a certain numerical invariant of link types, Trans. Amer. Math. Soc. 117 (1965), 387-422.

[M2] __, On the signature of links, Topology 9 (1970), 283-299.

[M3] K. Murasugi, Jones polynomials and classical conjectures in knot theory, Topology 26 (1987), 187-194.

[M4] _ Jones polynomials and classical conjectures in knot theory (II), Math. Proc. Cambridge Philos. Soc. 102 (1987), 317-318.

[M5] _ _ Jones polynomials of periodic link, Pacific J. Math. 131 (1988), 319-329.

[M6] _ On the chromatic degree of a graph (preprint).

[M7] _ - On the signature of a graph, C. R. Math. Rep. Acad. Sci. Canada 10 (1988), 107-111.

[M8] _ On the degree of Jones polynomials (preprint).

[M9] __, A property of Tutte polynomial (preprint).

[N] S. Negami, Polynomial invariants of graphs, Trans. Amer. Math. Soc. 299 (1987), 601-622.

[P1] J. H. Przytycki, $t_{k}$-moves on links, Contemporary Math., vol. 78, Amer. Math. Soc. Providence, R.I., 1988, pp. 615-656.

[P2] __, Positive knots have negative signature (preprint).

[Ro] D. Rolfsen, Knots and links, Publish or Perish, Berkeley, Calif., 1970.

[Ru] L. Rudolph, Non-trivial positive braids have positive signature, Topology 21 (1982), 325-327.

[Th1] M. B.Thistlethwaite, Spanning tree expansion of the Jones polynomial, Topology 26 (1987), 297-309

[Th2] __ Kauffman's polynomial and alternating links, Topology 27 (1988), 311-318.

[Th3] __, On the Kauffman polynomial of an adequate link, Invent. Math. 93 (1988), 285-296.

[Tr1] P. Traczyk, 10101 has no period 7: a criterion for periodic links, Proc. Amer. Math. Soc. (to appear).

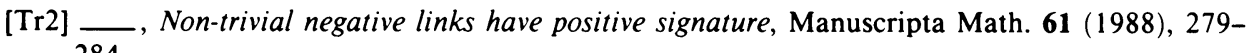
284.

[Tur] V. G. Turaev, A simple proof of Murasugi and Kauffman theorems on alternating links, L'Enseign Math. 33 (1987), 203-225.

[Tu] W. Tutte, A contribution to the theory of chromatic polynomials, Canad. J. Math. 6 (1953), 80-91.

[W] H. Whitney, 2-isomorphic graphs, Amer. J. Math. 55 (1933), 245-254.

Department of Mathematics, University of Toronto, Toronto, Canada M5S 1A1 